UC-712

Issued: April 1998

The Feed-Out Process: Rayleigh-Taylor and Richtmyer-Meshkov Instabilities in Thin, Laser-Driven Foils

D. Palmer Smitherman 


\section{DISCLAIMER}

This report was prepared as an account of work sponsored by an agency of the United States Government. Neither the United States Government nor any agency thereof, nor any of their employees, makes any warranty, express or implied, or assumes any legal liability or responsibility for the accuracy, completeness, or usefulness of any information, apparatus, product, or process disclosed, or represents that its use would not infringe privately owned rights. Reference herein to any specific commercial product, process, or service by trade name, trademark, manufacturer, or otherwise does not necessarily constitute or imply its endorsement, recommendation, or favoring by the United States Government or any agency thereof. The views and opinions of authors expressed herein do not necessarily state or reflect those of the United States Government or any agency thereof. 


\section{DISCLAIMER}

Portions of this document may be illegible electronic image products. Images are produced from the best available original document. 


\section{Acknowledgments}

Special thanks are extended to Dr. Glen Magelssen of Los Alamos National Laboratory (LANL) for his willingness to take on the responsibility of a graduate student. Gratitude is also extended to Nelson Hoffman of LANL for the many hours of educational discussions, and to the inertial fusion program at LANL for supporting this research. The Department of Energy funded my education through the Nuclear Engineering and Health Physics Fellowship, administered by the Oak Ridge Institute for Science and Education, for which I am indebted to them. The research described in this dissertation was financed by the Department of Energy under contract W-7405-ENG-36. 



\section{Table of Contents}

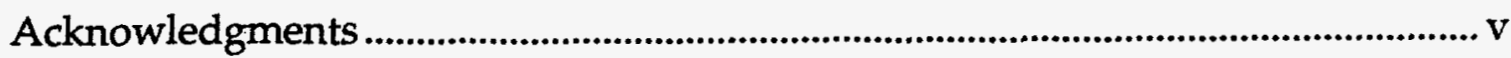

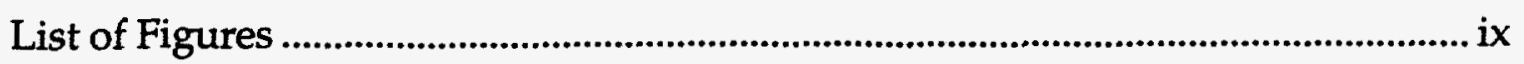

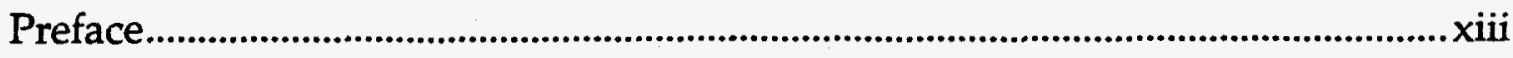

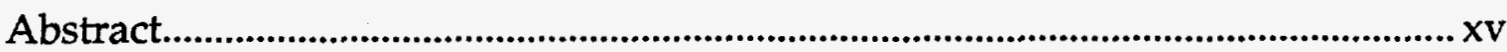

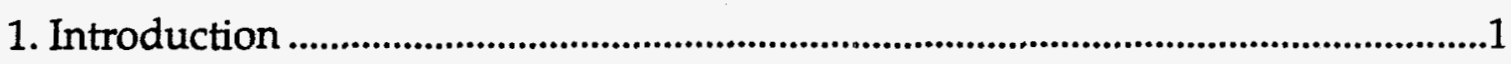

1.1 Inertial Confinement Fusion (ICF) ...................................................

1.2 Purpose and Approach of Dissertation ................................................11

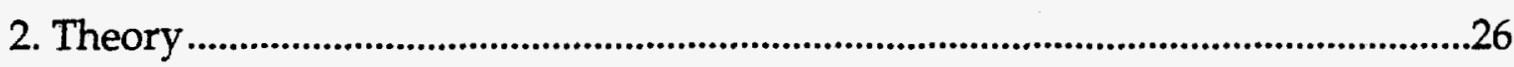

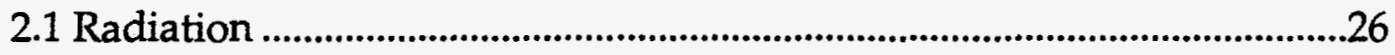

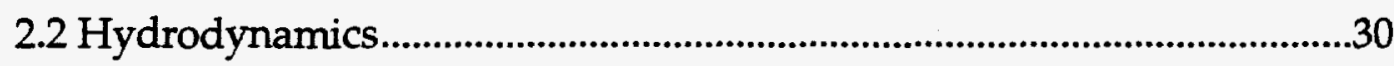

2.2.1 Introduction to the Rayleigh-Taylor Instability ........................35

2.2.2 Single Mode Rayleigh-Taylor Growth ....................................39

2.2.3 Multiple Mode Rayleigh-Taylor Growth ...............................49

2.2.4 The Rayleigh-Taylor Instability in Stratified Fluids..................58

2.2.5 The Richtmyer-Meshkov Instability .....................................65

2.2.6 The Richtmyer-Meshkov Instability in Stratified Fluids..........73

2.2.7 Atmospheric Type Modes.........................................................80

2.2.8 Differential Acceleration .......................................................8

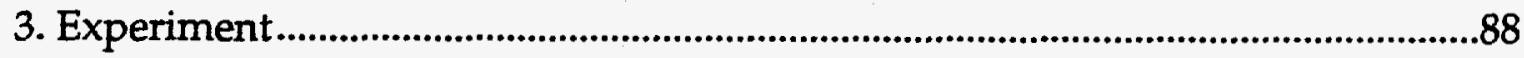

3.1 Related Experimental Work by Others ..............................................88

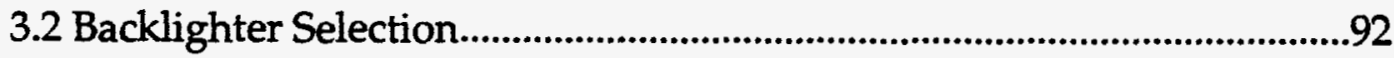


viii

3.3 Target Fabrication.........................................................................................99

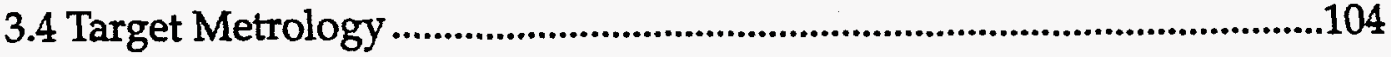

3.5 Target Fielding and Diagnostics ................................................................107

4. Computation with LASNEX.....................................................................................117

5. Computational and Experimental Results .............................................................122

6. Conclusion ....................................................................................................................159

Appendix: Details of Individual Shots .....................................................................165

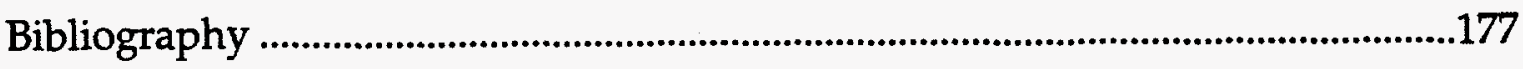




\section{List of Figures}

Figure 1-1: Indirect and Direct Drive Inertial Fusion.....................................................5

Figure 1-2: Direct drive ICF capsule ................................................................................7

Figure 1-3: Instability time history in ICF capsules ......................................................10

Figure 1-4: Package Time Histories ..............................................................................13

Figure 1-5: Experimental Setups for Face-on and Side-on Radiography .................17

Figure 1-6: The 2.2 ns (PS-26) and a 4.5 ns (PS-35) Drive Pulses..................................21

Figure 1-7: Packages That Were Shot .............................................................................22

Figure 2-1: Time History of Rayleigh-Taylor Instability...............................................36

Figure 2-2: Ablative Stabilization.......................................................................................47

Figure 2-3: Mode Coupling...............................................................................................55

Figure 2-4: Stratified Fluid Layers. ...................................................................................60

Figure 2-5: Riemann Problem.......................................................................................68

Figure 2-6: Typical Richtmyer-Meshkov Growth Rate ...............................................72

Figure 2-7: Sketch of Normalized Growth Rate...............................................................77

Figure 2-8: Atmospheric Type Modes ......................................................................83

Figure 2-9: Differential Acceleration ...........................................................................87

Figure 3-1: Vapor Deposition of $\mathrm{Al}$ on $\mathrm{Cu}$ Substrate....................................................98

Figure 3-2: Hohlraum with Batwings............................................................................100

Figure 3-3: Side View of Target Positioned in NOVA

Target Chamber. Face-on Radiography .................................................101

Figure 3-4: Additional Views of Face-on Radiography Target .................................102 
Figure 3-5A: Side-on Radiography ............................................................................103

Figure 3-5B: Side-on Radiography...........................................................................104

Figure 3-6: Section View of the NOVA Target Chamber .............................................108

Figure 3-7: Operation of X-ray Camera..........................................................................109

Figúre 3-8: Bragg Angle and Section View of Crystal Spectrometer .......................113

Figure 3-9: Streak Camera Operation................................................................................114

Figure 5-1: Fourier Amplitudes and Surface Perturbations......................................125

Figure 5-2: Fourier Amplitudes of the $86 \mu \mathrm{m}$ Al Foil, PS-26, Fe Backlighting .....126

Figure 5-3: Side-on Data of the $35 \mu \mathrm{m} \mathrm{Al}$ and $86 \mu \mathrm{m}$ Al Foils, PS-26.....................128

Figure 5-4: Fourier Amplitudes of the $35 \mu \mathrm{m}$ Al Foil, PS-26, Ti Backlighting ......130

Figure 5-5: Density Contours of the $35 \mu \mathrm{m}$ Al Foil, PS-26........................................131

Figure 5-6: Fourier Amplitudes of the $84 \mu \mathrm{m} \mathrm{Al} / 10 \mu \mathrm{m}$ Be Foil,

PS-26, Fe Backlighting

Figure 5-7: Fourier Amplitudes of the $32 \mu \mathrm{m} \mathrm{Al} / 10 \mu \mathrm{m}$ Be Foil,

PS-26, Ti Backlighting .136

Figure 5-8: Fourier Amplitudes of the $32 \mu \mathrm{m} \mathrm{Al} / 10 \mu \mathrm{m}$ Be Foil,

PS-26, Sc Backlighting 137

Figure 5-9: Fourier Amplitudes of the $50 \mu \mathrm{m}$ Al Foil, PS-35, V Backlighting.......140

Figure 5-10: Density and Vorticity Contours of the $50 \mu \mathrm{m} \mathrm{Al} \mathrm{Foil,} \mathrm{PS-35}$ .141

Figure 5-11: Feed-out from Differential Acceleration and Interface Coupling ....148

Figure 5-12: Feed-out from Interface Coupling with a Density Jump 150

Figure 5-13: Fourier Amplitudes of the $35 \mu \mathrm{m}$ Al Foil, PS-26, Sc Backlighting....154 
Figure 5-14: Face-on Data of the $35 \mu \mathrm{m}$ Al Foil Showing Frequency Doubling...155 Figure 5-15: Fourier Amplitudes of the $35 \mu \mathrm{m}$ Al Foil, PS-35, Sc Backlighting....158 



\section{Preface}

This dissertation presents a computational and experimental study of feed-out, a hydrodynamic phenomenon that is important to inertial confinement fusion capsule implosions. The computational work was conducted at Los Alamos National Laboratory (LANL), in Los Alamos, New Mexico, with experiments performed at the NOVA laser facility located at the Lawrence Livermore National Laboratory in Livermore, California.

I was responsible for designing the experiments and the computational investigation of the phenomenon. Fielding the experiments was the responsibility of Robert Chrien, a staff scientist at LANL. I assisted Chrien during all but three of the shots, performing tasks such as metrologizing the targets, taking part in diagnostic alignment, and adjusting crystal angles in the spectrometers. Experimental questions involving code predictions, such as the $\mathrm{X}$-ray camera timings, were decided by both Chrien and myself. Chrien analyzed the data and arranged for target fabrication, a service provided by the laboratory.

The first chapter of the dissertation provides an introduction to inertial fusion and an overview of the campaign. Chapter two is a review of relevant theories from the literature, while chapter three presents a more detailed account of the experimental procedure than found in chapter one. A discussion of computational considerations and the code used is found in chapter four.

Chapters two, three, and four are important. Science uses an iterative 
xiv

process of theory and experiment to converge on the solution to a physical question. Theory provides physical understanding and may be used to predict the outcome of an experiment. Experiment is used to help confirm theoretical predictions. If the two answers disagree, it does not necessarily mean that theory is incorrect. Even if theory and experiment do agree, they could both be incorrect for different reasons. The rigor of the experimental and theoretical procedures is just as significant as agreement or disagreement between their respective results. When faced with a discrepancy between the two, the scientist must understand the limitations of each to resolve the problem. For example, could there have been a consistent machining error during target fabrication, or were the opacity tables used in the calculation less than optimal?

Computational and experimental results are discussed in chapter five. Details describing individual shots are found in the appendix including laser energies and diagnostic settings. The appendix also contains lessons learned while fielding the shots, such as which filter worked best with the $X$-ray cameras.

The terms "package," "foil," and "target" are frequently encountered in this work. "Package" and "foil" both refer to the planar slab of material placed on the side of the hohlraum, which was the subject of the hydrodynamic experiments. The target is collectively the package, hohlraum, backlighter, radiation shields, and alignment wire, all of which were placed in the laser target chamber and were the "target" at which the laser was fired.

DPS 
THE FEED-OUT PROCESS:

\title{
RAYLEIGH-TAYLOR AND RICHTMYER-MESHKOV INSTABILITIES IN THIN, LASER-DRIVEN FOILS
}

by

D. Palmer Smitherman

\begin{abstract}
Eight beams carrying a shaped pulse from the NOVA laser were focused into a hohlraum with a total energy of about $25 \mathrm{~kJ}$. A planar foil was placed on the side of the hohlraum with perturbations facing away from the hohlraum. All perturbations were $4 \mu \mathrm{m}$ in amplitude and $50 \mu \mathrm{m}$ in wavelength. Three foils of pure aluminum were shot with thicknesses and pulse lengths respectively of $86 \mu \mathrm{m}$ and $2.2 \mathrm{~ns}, 50 \mu \mathrm{m}$ and $4.5 \mathrm{~ns}$, and $35 \mu \mathrm{m}$ with both $2.2 \mathrm{~ns}$ and $4.5 \mathrm{~ns}$ pulses. Two composite foils constructed respectively of 32 and $84 \mu \mathrm{m}$ aluminum on the ablative side and $10 \mu \mathrm{m}$ beryllium on the cold surface were also shot using the $2.2 \mathrm{~ns}$ pulse. X-ray framing cameras recorded perturbation growth using both face- and side-on radiography.

The LASNEX code was used to model the experiments. A shock wave interacted with the perturbation on the cold surface generating growth from a Richtmyer-Meshkov instability and a strong acoustic mode. The cold surface per-
\end{abstract}


xvi

turbation fed-out to the Rayleigh-Taylor unstable ablation surface, both by differential acceleration and interface coupling, where it grew. A density jump did not appear to have a large effect on feed-out from interface coupling. The RayleighTaylor instability's vortex pairs overtook and reversed the direction of flow of the Richtmyer-Meshkov vortices, resulting in the foil moving from a sinuous to a bubble and spike configuration. The Rayleigh-Taylor instability may have acted as an ablative instability on the hot surface, and as a classical instability on the cold surface, on which grew second and third order harmonics. 


\section{Introduction}

\subsection{Inertial Confinement Fusion (ICF)}

Inertial confinement fusion (ICF) is a process by which a small sphere of hydrogen, the capsule, is imploded using either a laser or particle beam [Duderstadt]. The hydrogen reacts to form nuclides with a lower total potential energy, releasing energy in the process. The hydrogen is usually a 1:1 mixture of deuterium and tritium (DT) because of the higher cross section of this reaction compared to other fusion reactions. The DT reaction is

$$
D+T \rightarrow \alpha(3.5 \mathrm{MeV})+n(14.1 \mathrm{MeV}) .
$$

Fusion cross sections are orders of magnitude smaller than their uranium fission counterparts, but reaction rates are proportional to the density of each ion species being burned and roughly scale to the fourth power of temperature. The ICF approach is to confine the capsule with its own inertia for a very short time, 100's of picoseconds, but produce extremely high reaction rates with high densities and temperatures on the orders of $10^{3}-10^{4}$ times solid-state density and $10 \mathrm{keV}$ respectively.

Historically, there have been three motivations for the study of ICF. They are commercial energy production, nuclear weapons physics, and pure scientific research. In the pursuit of each of these goals, ICF possesses advantages and disadvantages over the alternatives.

ICF offers the possibility of a more environmentally benign commercial 
energy source than light water reactors or coal-fired plants. It would produce much less radioactive waste than conventional fission reactors, and no greenhouse gases. However, the storage of large amounts of tritium on site could represent an airborne radiation hazard to the local populace. ICF technology would have to advance greatly, at high initial investment, before an ICF power station could economically produce electricity.

The major competitor with fusion from a technical standpoint is a breeder reactor design with an accompanying waste transmutation reactor to destroy the long-lived daughter products and actinides. An equivalent alternative to the breeder-transmutation scheme would be designing a burner, which is a breeder that burns long-lived radioactive waste. Advanced breeders could be much safer and generate less long-lived radioactive waste than conventional reactors, but still more than ICF. The breeder would be much less expensive than ICF to develop, and have a high probability of success, as it represents a perturbation on presently operating technology. Present research focuses on DT fusion, but with the limited supply of lithium for tritium breeding, DT power might only represent a few centuries of electricity at current demand rates. If the more difficult DD fusion reaction could be harnessed, this achievement might represent thousands of years of power, but so could the breeder if uranium was mined from seawater. ICF for energy production would require a shift in the present research orientation from lasers to the more efficient ion beams, but even so, electricity from ICF looks doubtful. 
With the present ban on nuclear weapons testing, ICF has taken on a more critical role in the understanding of weapons physics. Weapons science is often stated as a significant reason for building the National Ignition Facility (NIF), a 1.8 MJ ICF laser facility with which researchers hope to achieve ignition in ICF capsules. The NIF would be one of several facilities on which weapons physics experiments would be conducted. It appears significant that some of those designing weapons do not view the NIF and ignition as fundamental to weapons physics, and suggest that other experiments, which are more cost effective, should be considered in place of NIF. Another difficulty facing the NIF is that the exact purpose of ignition has not yet been clearly defined by the ICF community.

The last motivation for ICF research is pure science. Experiments can be designed to yield relevant data on astrophysical phenomena, such as instabilities in supernova. Equations of state in extreme regions of parameter space can be compared to theory and the interaction of strong radiation fields with matter investigated.

The most important machine for ICF research is the driver. The ICF driver provides the energy needed to compress the capsule and is usually a laser or particle beam. The driver may either directly interact with the capsule, as in direct drive, or with a metallic structure surrounding the capsule called a hohlraum, as in indirect drive [Hogan], see Figure 1-1. The hohlraum converts the incident driver radiation into $X$-rays, which illuminate the capsule and compress it. 
Figure 1-1: Indirect and Direct Drive Inertial Fusion

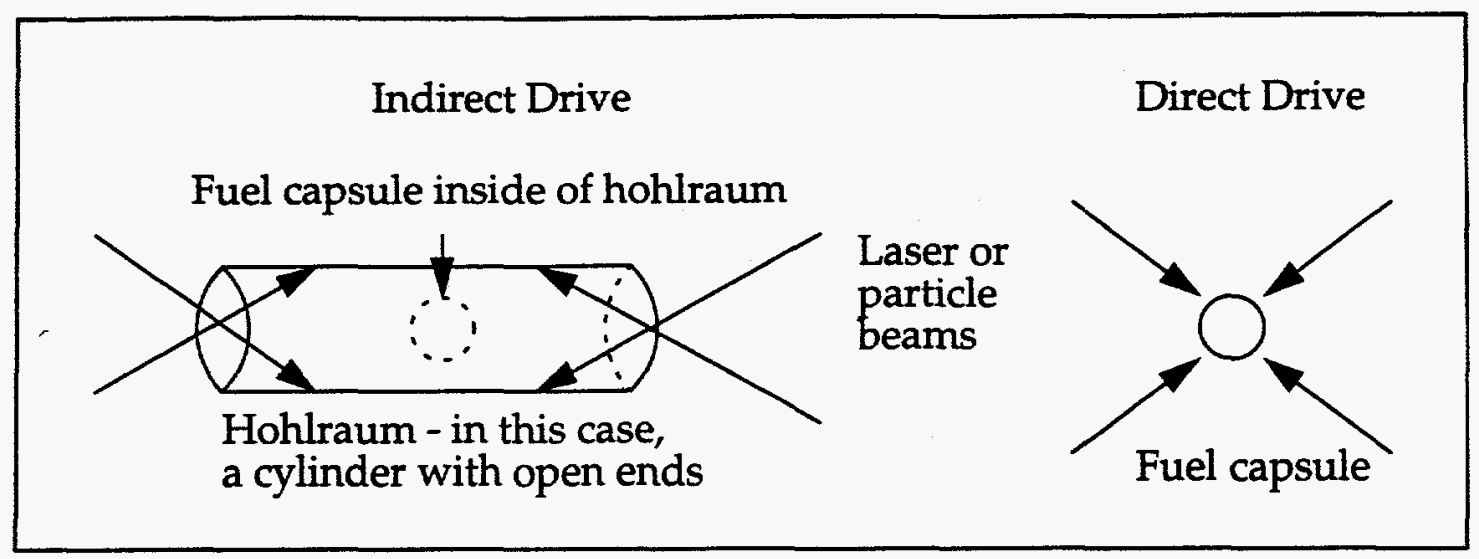

Hohlraums may be cylindrical, tetrahedral, or of many other shapes and are usually on order of one or two millimeters in size. Indirect drive produces a much more uniform radiation field with lesser laser beam quality than direct drive, thereby increasing capsule stability. The penalty is an energy loss in the conversion of laser energy to X-rays.

As ICF drivers, ion beams and lasers have different advantages [Hogan]. It is difficult to focus particle beams to the small size required to directly drive an inertial fusion capsule, unlike a laser driver. Lasers only have efficiencies of $0.25-8 \%$, compared to the $20-30 \%$ for ion beams. Ion beams can also easily produce the megajoules of energy needed for ignition and burn, while it is difficult to produce this much energy with a laser. Everything considered, the Department of Energy's Fusion Policy Advisory Committee judged heavy-ion accelerators to be the leading candidate for a reactor driver. Currently, however, ICF research is using laser drivers because they are inexpensive compared to heavy ion facilities, and offer the potential to learn a great deal about capsule physics in the shortest 
time. Some of this information would be transferable to an ICF ion facility.

The capsule is usually a sphere composed of two types of material, the hydrogen fuel on the inside, and an ablator on the outside. During the implosion, the pusher confines the fuel through inertia and converts the energy of the driver into mechanical work on the fuel. The pusher in this sense can be both the ablator as well as cold fuel adjacent to the ablator. The object is to first compress the capsule along a low adiabat to high density, then produce a small hot region at the center using converging shocks. The cold dense fuel adjacent to the "hot spot" traps alpha particles from the fusion process. In this way, the nuclear burn propagates through the capsule, and a much larger percentage of the energy needed to heat the capsule to fusion temperatures comes from fusion itself instead of the driver [Lindl].

The direct drive ICF capsule can be divided into three principal regions [Duderstadt] as shown in Figure 1-2. The most exterior is the energy deposition region, in which the laser light travels. The laser deposits energy here by inverse bremsstrahlung and resonance absorption, which is the coupling between electron plasma waves and light. Stimulated Raman scattering and stimulated Brillouin scattering are laser-plasma interactions that partially reflect the laser energy, and reduce absorption in this region. The limit of the energy deposition region is the critical density, where the plasma frequency becomes larger than the light frequency. Driver radiation does not penetrate past this point. 
Figure 1-2: Direct Drive ICF Capsule

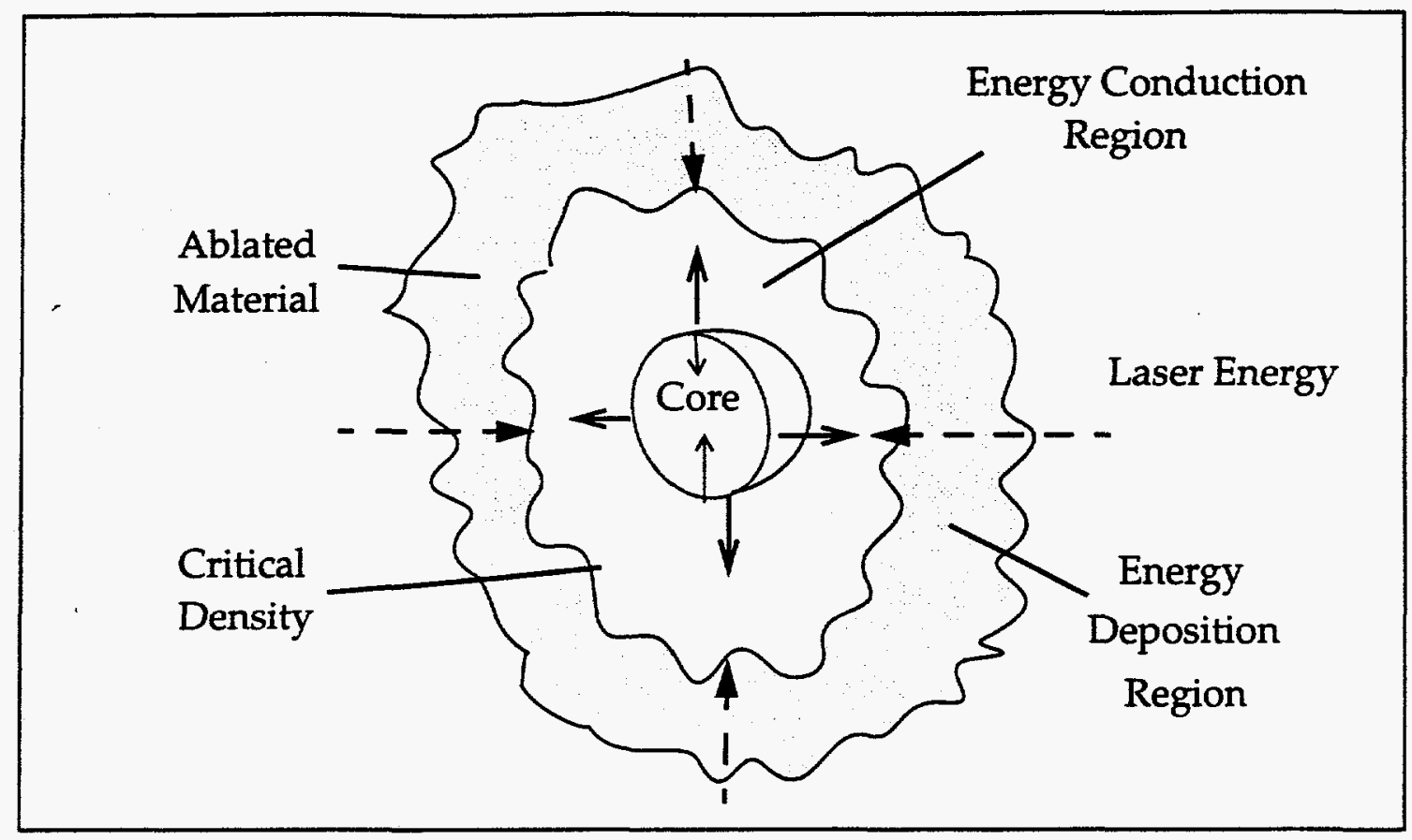

In the energy conduction region, material streams off the capsule surface and into the energy deposition region. Energy is transported by radiation and electron thermal conduction from the critical density to the ablation surface. Electron thermal conductivity is found to be much lower here than would normally be expected. The generation of large local magnetic fields and two-stream instabilities, generated from the heated electrons moving toward the capsule and the return current, can significantly reduce heat conduction by reducing the electron mobility.

The third region is composed of the core, or unablated DT material. By dividing the fuel disassembly time by the burn rate one finds the condition for a good burn in which a significant fraction of the fuel is consumed, tens of percent. 
For a good burn, the integral of the fuel density along a radial line emanating from the center of the capsule to the pusher/fuel interface must be greater than 1 $\mathrm{g} / \mathrm{cm}^{2}$. Frequently this integral is termed "pr." The range of a $3.5 \mathrm{MeV}$ alpha is about $0.5 \mathrm{~g} / \mathrm{cm}^{2}$, so a pr greater than $1 \mathrm{~g} / \mathrm{cm}^{3}$ also insures good fusion energy deposition in the fuel. It is not advantageous to continue to increase pr without limit. It takes greater drive energy to compress to a higher $\rho r$, but with diminishing returns in the fraction of fuel burned. An optimal value for DT fusion is about $3 \mathrm{~g} / \mathrm{cm}^{2}$, corresponding to roughly $30 \%$ fuel burn [Duderstadt].

For indirect drive, the physics of the laser interaction with the hohlraum is much the same as the energy conduction and absorption regions of a direct drive capsule. The hohlraum generates $X$-rays in the $100-200 \mathrm{eV}$ range, which ablate the capsule, and interact directly with the surface throughout the implosion.

Of great importance to a successful implosion is symmetry. The hydrodynamics of an ICF capsule system is analogous to a basketball sitting on the point of a pencil. As long as the symmetry is perfect, the system is stable, but if there are any perturbations from this ideal state, the system quickly becomes unstable. Hydrodynamic instabilities cause any asymmetry on the capsule or in the DT to grow, resulting in mixing of the hot and cold areas of the fuel, mixing of the ablator and fuel, or complete disassembly of the capsule in extreme cases. In any event, much less of the fuel burns than if the implosion were perfectly symmetric. There are always perturbations from target fabrication on the surface of 
the ablator and on the inside surface of the frozen fuel layer, see Figure 1-3. Present technology can limit these abnormalities to within a few micrometers in amplitude, but they are still a threat to proper implosion symmetry. Asymmetries in the radiation driving the capsule can create perturbations on the capsule surface by pushing harder on one region than another, in both indirect and direct drive. For example, if there is an imbalance in the power of the laser beams, the implosion will not be symmetric. For direct drive, the field intensities of the laser beams are variable in space and time, resulting in a "foot print" on the surface of the capsule early in time.

The two most important instabilities are the Rayleigh-Taylor and Richtmyer-Meshkov instabilities. After the drive pulse is turned on, the RayleighTaylor instability, caused by a low density fluid pushing on a higher density fluid, results in growth of perturbations on the ablation surface. A shock wave begins to move through the capsule. When it reaches the inside surface of the DT ice, it interacts with the ice perturbations, generating a Richtmyer-Meshkov instability. As the ablator surface perturbations grow, they feed into the fuel, adding to the growth from the Richtmyer-Meshkov instability. When the shock arrives at the center of the capsule, it produces a low density, high temperature hot spot in the fuel. This light material begins to push outward on the converging colder material, eventually stagnating the compression. A Rayleigh-Taylor instability now develops during this deceleration phase on the interface between the hot and cold sections of the fuel, resulting in even greater internal perturbation growth. If the 
Figure 1-3: Instability Time History in ICF Capsules.

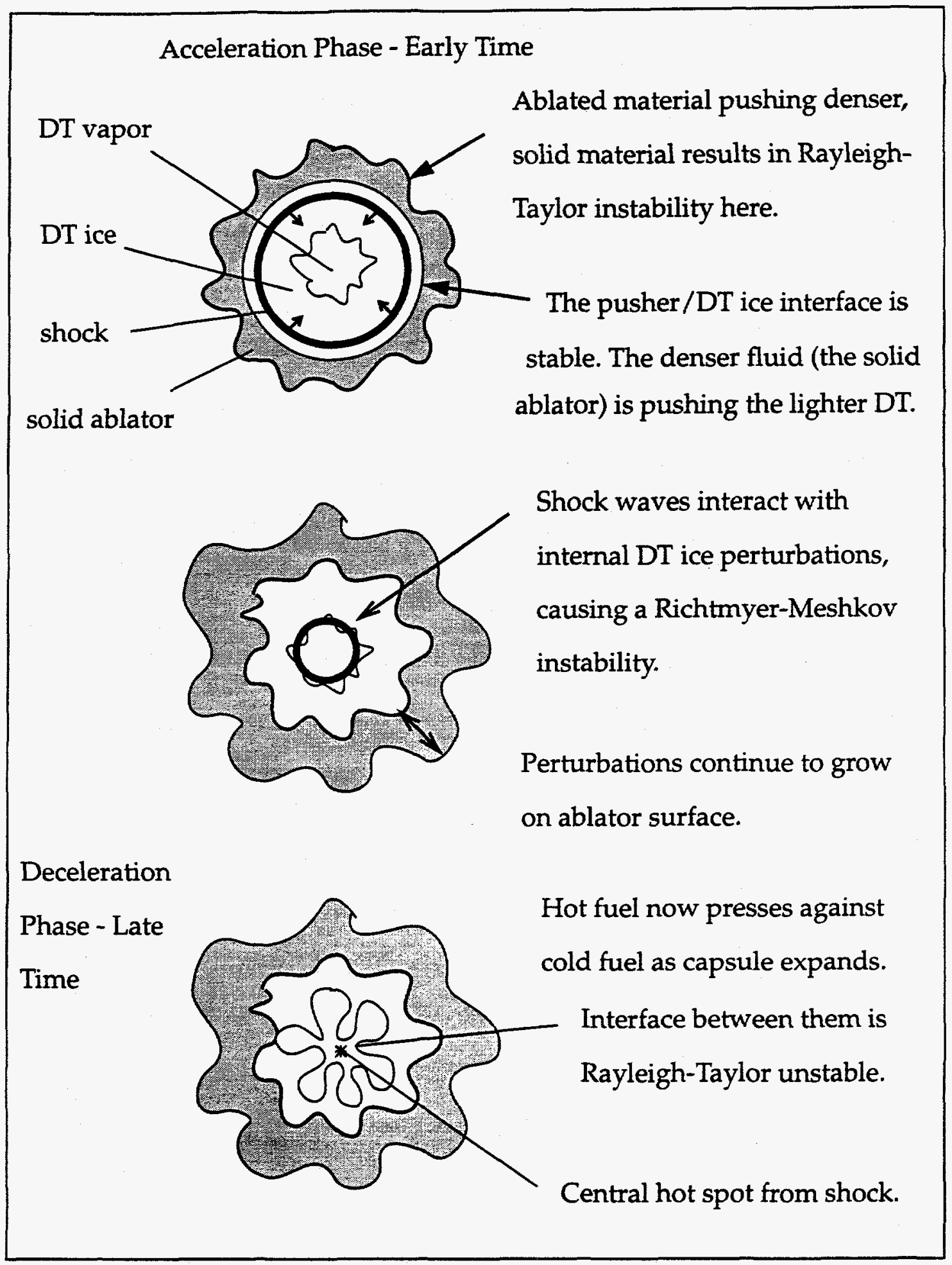


internal perturbations are too large, the hot spot cools and the capsule does not ignite. As of this writing, the ICF community has not yet achieved ignition in large part because of these reasons.

\subsection{Purpose and Approach of Dissertation}

To determine the effect ice perturbations of various amplitudes have on indirect drive NIF capsule performance, a robustness study was conducted by Hoffman and Wilson [Krauser] using the two-dimensional radiation-hydrodynamics code LASNEX, described in chapter 4 . Hoffman calculated capsules with plastic ablators, while Wilson's capsules used beryllium. They both slowly increased the amplitude of the internal perturbations while giving the exterior perturbations and radiation drive perfect symmetry. As the amplitude increased, the neutron yields decreased.

The calculations showed a dramatic difference between the yield vs. roughness curves of the plastic and beryllium capsules. The plastic capsule fell to a zero yield at a roughness of $2 \mu \mathrm{m}$ RMS, while the beryllium fell to a zero yield at a roughness greater than $8 \mu \mathrm{m}$ RMS. The beryllium design was obviously much more resistant to perturbations on the ice than the plastic, but why?

Why beryllium was better was a difficult question to answer. Beryllium and plastic have different material properties and different equations of state. In addition, both materials were doped with high opacity elements. The shells were different masses. The plastic drive pulse was hotter than the beryllium pulse, but 
the beryllium pulse was a little longer. At the end of the radiation pulse, the ablation front was closer to the DT in the plastic design than in the beryllium. To complicate matters further, Wilson assumed a Planckian drive spectrum for the beryllium capsule, while Hoffman included the high energy gold M-band in the spectrum for the plastic capsule calculations. The plastic capsule thus experienced greater preheat than the beryllium. Despite all of these differences, one important mechanism was suspected of creating the difference in the yield curves.

Sometime after perturbations begin to grow on the inside of the capsule, they also appeared on the outside of the capsule and begin to increase in amplitude. As they increased in amplitude on the ablation surface, they grew back into the capsule, eventually reaching the interior with an amplitude much larger than the original internal perturbations. The hydrodynamic communication of an interior perturbation to the exterior and its subsequent growth was dubbed "feed-out."

Figure 1-4 shows the sequence of events that occur in a planar slab during the feed-out process and is representative of what would occur in an ICF capsule with a smooth outer surface and perturbed interior. After the drive is turned on, the hohlraum radiation began to ablate the smooth side of the package, sending a shock wave through the material and compressing the package. A Richtmyer-Meshkov instability is created by the shock when it interacts with the perturbation on the back of the foil, causing the perturbations to phase-invert and grow. An acoustic mode is established behind the cold surface as part of the Rich- 
tmyer-Meshkov flow field. A rarefaction wave, created by the reflection of the shock off the back side, began moving toward the ablation surface and is the leading edge of the Richtmyer-Meshkov flow field. After the rarefaction reaches the hot side, the ablation surface is "aware" of the perturbations on the back. A strong Raýleigh-Taylor instability located at the ablation surface then causes the pert-

Figure 1-4: Package Time Histories

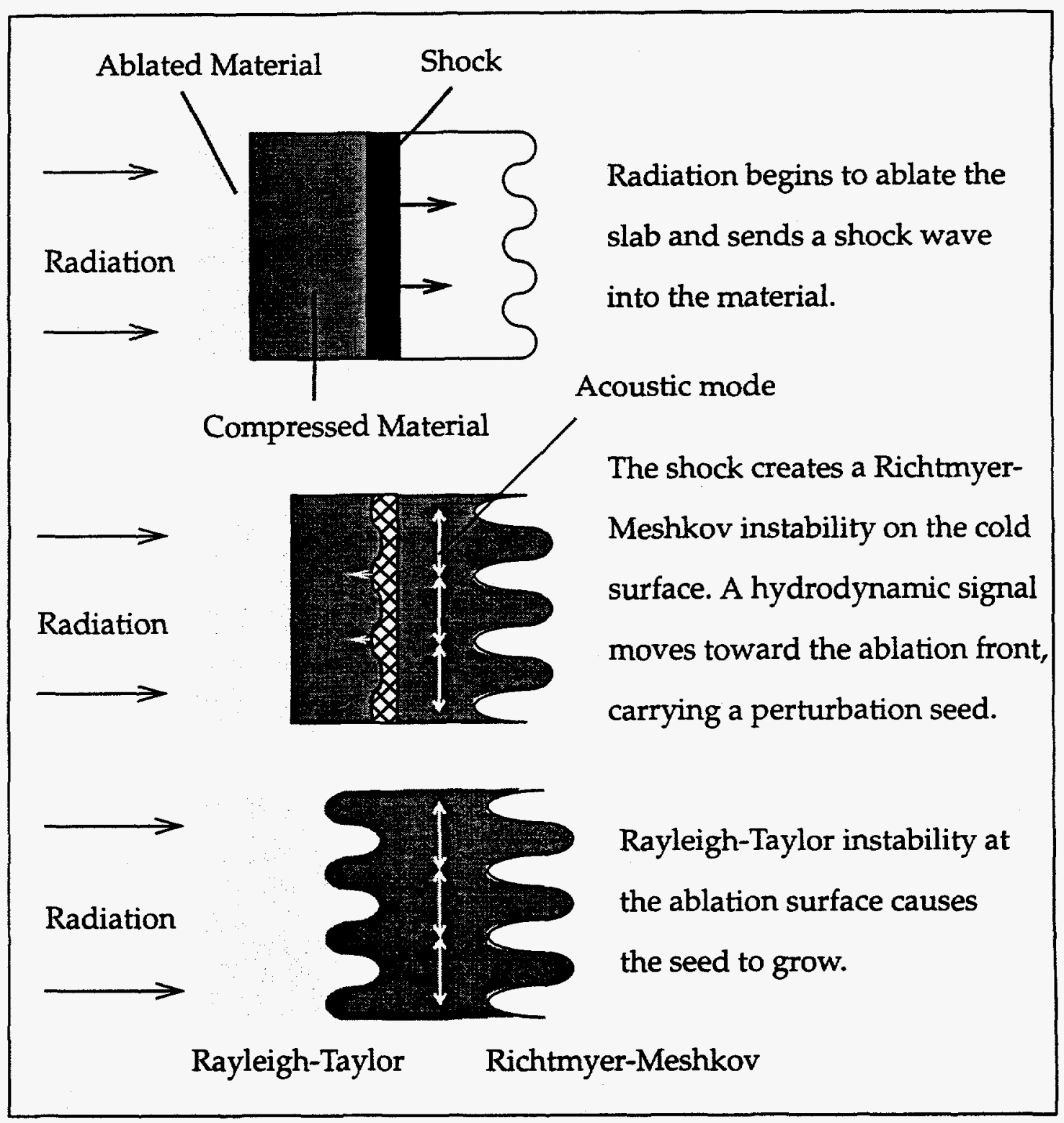


urbations on the hot side to grow. The Rayleigh-Taylor instability can only exist as long as the drive is on, but the Richtmyer-Meshkov instability is independent of the drive. As long as the foil is relatively thick, the Richtmyer-Meshkov and Rayleigh-Taylor instabilities grow independently. After radiation had burned through a significant amount of the foil, the two instabilities begin to interact. At this point, the perturbations on the ablation surface have fed-back through the foil and begin to perturb the cold surface.

At least two mechanisms are believed to seed the Rayleigh-Taylor instability on the ablation surface. The flow field of the Richtmyer-Meshkov instability on the cold surface could carry the perturbation back to the ablation surface. This mechanism is frequently referred to in this document as interface coupling. Alternately, perturbation growth on the surface could be seeded by a differential acceleration effect. There is less mass under the valleys of the initial perturbations than under the peaks. If the radiation drive was uniform, it would push the lower mass regions faster than the higher mass regions, resulting in a perturbation, and growth. Either or both could act as a seed for the Rayleigh-Taylor instability on the ablation surface.

Feed-out was immediately recognized as a serious threat to ICF capsules. The ablation surface is a highly unstable region. Perturbations grow rapidly on the ablation surface and can easily grow large enough to prevent the capsule from imploding properly. The exterior of the capsule may be polished to a roughness of about $10 \mathrm{~nm}$ RMS, but the interior of the DT ice perturbations cannot be 
smoothed with present technology to less than about $0.5 \mu \mathrm{m}$ RMS. During most of the time the capsule is imploding, the interior instabilities are not as severe as those on the ablation surface. One might conclude that the adverse effects of large internal perturbations would be mitigated by the short duration of the internal instabilities. The calculations of Wilson and Hoffman showed this may not to be the case. The larger internal perturbations coupled with the strong, long-duration ablation front instabilities during the implosion through feed-out, degrading the yield to unacceptable levels.

Differences in the feed-out of beryllium and plastic was suspected as the reason for the difference in the beryllium and plastic yield curves. Although the initial thicknesses of the beryllium and plastic ablators in the NIF capsule design were about the same, the beryllium ablated slower. As a result, the beryllium became thicker than the plastic in the implosion. The greater distance between the cold surface and the ablation front could have reduced the size of the Rayleigh-Taylor seed from feed-out in the beryllium. In addition, the greater distance could have also inhibited the Rayleigh-Taylor growth from feeding-back into the interior.

There was an additional important difference between the plastic and beryllium designs. The plastic had a density of about $1.0 \mathrm{~g} / \mathrm{cm}^{3}$, while the beryllium had a density around $1.85 \mathrm{~g} / \mathrm{cm}^{3}$. This meant that there was a much larger density jump at the intersection of the DT ice and the ablator in the beryllium than in the plastic. The ice had a density of $0.25 \mathrm{~g} / \mathrm{cm}^{3}$. Was it possible that a larger 
density jump could be a greater barrier to interface coupling between the cold and hot surfaces? If the greater density jump was a barrier, the Rayleigh-Taylor seed from feed-out would have been smaller in beryllium than plastic.

In order to understand more clearly the reason for the superiority of beryllium over plastic, it was necessary to learn more about feed-out. Planar experiments were performed on the NOVA laser facility at Lawrence Livermore National Laboratory to experimentally confirm that feed-out exists and to study its dependence on thickness and density jump. Planar slabs of material very similar to the one displayed in Figure 1-4 were accelerated with radiation from a hohlraum. The slab is frequently referred to in this paper as the "package" or "foil." It was positioned in a small hole on the side of a hohlraum, so only the side facing inward, the hot side, received radiation, as shown in Figure 1-5. The hot side was smooth, while the side facing away from the hohlraum, the cold side, had a machined sinusoidal perturbation.

The two experimental setups used in the feed-out shots, face-on and side-on radiography, are shown in Figure 1-5.Notice the upper diagram displaying the face-on configuration. High energy $X$-rays were generated by firing two of NOVA's ten beams onto a small metal disk, the backlighter, on the opposite side of the hohlraum from the package. The specific energy of these X-rays was determined by the element comprising the backlighter but was always between 4.3 $8.3 \mathrm{keV}$. There were holes in the hohlraum just large enough to allow passage of the $\mathrm{X}$-rays through the hohlraum and through the package, after which they were 
Figure 1-5: Experimental Setups for Face-on and Side-on Radiography

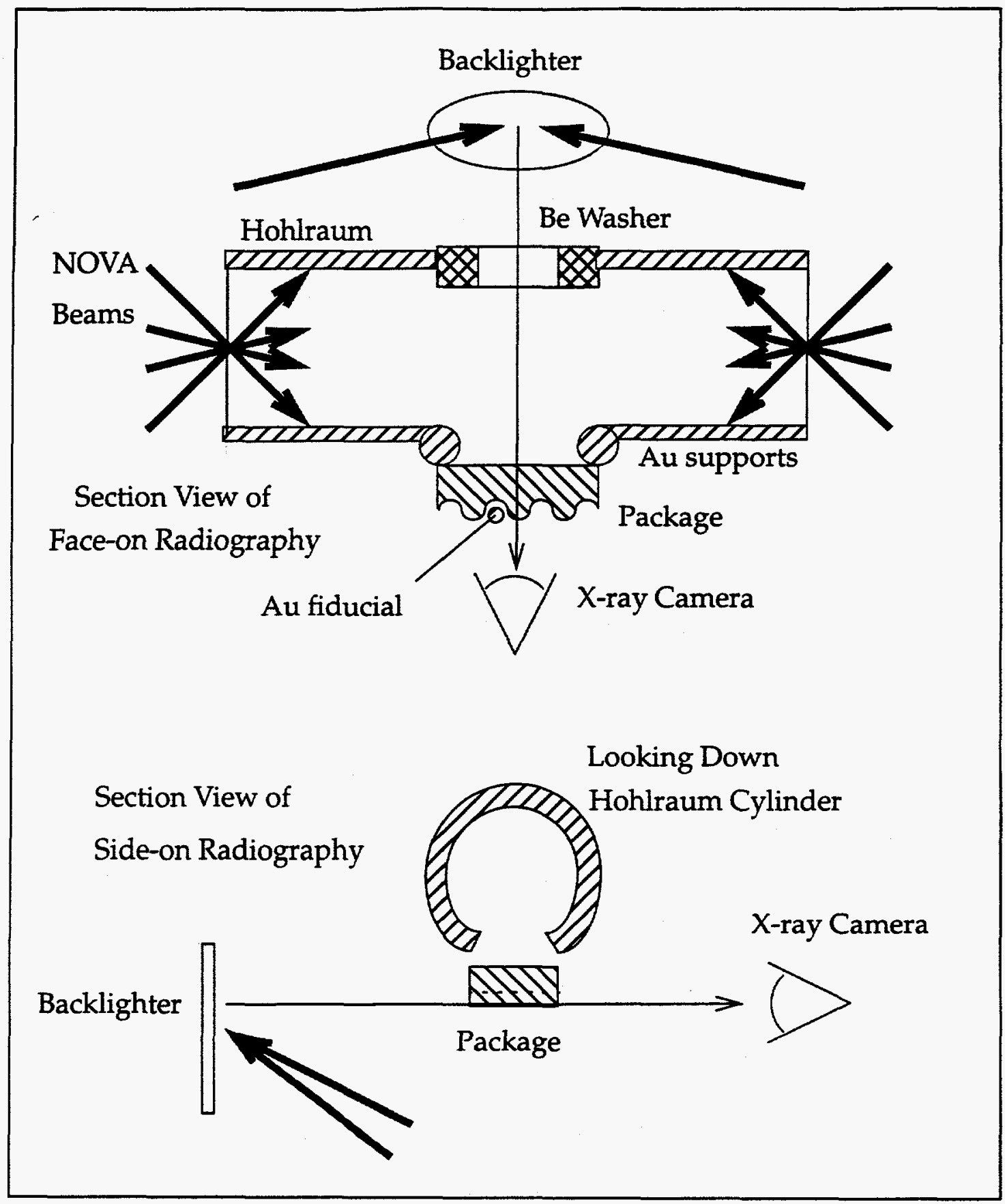


photographed with an X-ray camera. A beryllium washer was glued to the hole in the hohlraum on the backlighter side. Without the washer, gold vapor from the hohlraum would fill in the small hole. Gold was not transparent to the high energy $X$-rays from the backlighter, but beryllium was. A small gold wire was placed in the trough of the machined perturbations as a fiducial for phase changes. The line of sight of the camera was perpendicular to the plane of the package.

In face-on radiography, $\mathrm{X}$-rays from the backlighter were attenuated more by passing through peaks in the perturbations of the package than by passing through the troughs. The difference in attenuation produced dark areas on the film from the valleys of the perturbations, and lighter areas from the perturbation peaks. The data could then be Fourier-analyzed to reveal the growth of the first, second, and third harmonics with time.

Face-on radiography quantitatively revealed perturbation growth, but did not show the location of the perturbations. They could be on the hot surface, the cold surface, or even in the center of the foil. Side-on radiography, also shown in Figure 1-5, revealed the location of the perturbations by placing the line of sight of the camera in the same plane as the package, and parallel to the initial perturbations. The backlighter likewise was repositioned to illuminate the package from the side. Package surfaces adjacent to and away from the hohlraum were thus simultaneously visible. Side-on radiography data was much more difficult to compare to calculations than face-on and only provided qualitative information. 
In addition to the $\mathrm{X}$-ray camera observing the package, two other diagnostics collected data on the backlighter to make certain it was performing as expected. One was an $\mathrm{X}$-ray camera observing the side opposite laser irradiation. Low energy parts of the spectrum were filtered out passing through the disk, so only the higher energies used for the package diagnostic created an image. The purpose of this diagnostic was to ascertain when the backlighter was turned on and off, and how bright the source was qualitatively. A spectrometer connected to a streak camera observed the laser-illuminated surface, and showed the relative strengths of the high energy lines used for backlighting.

The X-ray drive was generated by focusing eight of NOVA's ten beams into the hohlraum. The hohlraums were constructed of gold due to its efficient conversion of the laser energy into X-rays. The M-band of gold always produced some $2-4 \mathrm{keV} X$-rays in addition to the thermal radiation. A total energy of 24 or $25.6 \mathrm{~kJ}$ in the hohlraum was requested on each shot, but in reality the energy varied from about $18-27 \mathrm{~kJ}$.

Experimental design was a two-step process. First, a package with the desired characteristics was found by running various LASNEX simulations. A postprocessor, TDG, was then run to simulate data from the X-ray camera. The task for face-on radiography was to find the backlighter energy that would most effectively show the perturbations. For side-on radiography, an energy had to be selected that would show the density contours with the greatest perturbations. To some extent, these selections were based on trial and error. A detailed discussion 
of backlighter selection for both face- and side-on geometries is presented in section 3.2 .

In addition to designing the experiments, LASNEX was used to computationally study feed-out and to provide a better understanding of what was occurring in the shots. The computational results were compared to experimental data and conclusions drawn from both sets of results. The campaign was as much a computational as experimental effort.

For this initial investigation of feed-out, the desire was to observe experimental conditions ranging from weak to strong coupling of the ablation and cold surfaces. The level of coupling was adjusted by changing the thickness and pulse length, see Figures 1-6 and 1-7. Three cases were studied.

The first case was an $86 \mu \mathrm{m}$ thick, aluminum foil. The thickness was large enough so that there was only weak coupling between the ablation and cold surfaces. The shock hit the rear surface of the "thick" package about the same time the drive was turning off, so only the Richtmyer-Meshkov instability was present. The thick package offered the opportunity to observe how the seed for the Rayleigh-Taylor developed.

The second foil had a thickness of $35 \mu \mathrm{m}$ of aluminum. The shock from the drive pulse hit the rear surface of the "thin" package after about 1ns, at which time the drive had burned through much of the foil. The foil thickness was thus much less than the wavelength, resulting in strong and fast coupling of the Rayleigh-Taylor instability with the Richtmyer-Meshkov instability. 
Figure 1-6: The 2.2 ns (PS-26) and 4.5 ns (PS-35) Laser Drive Pulses
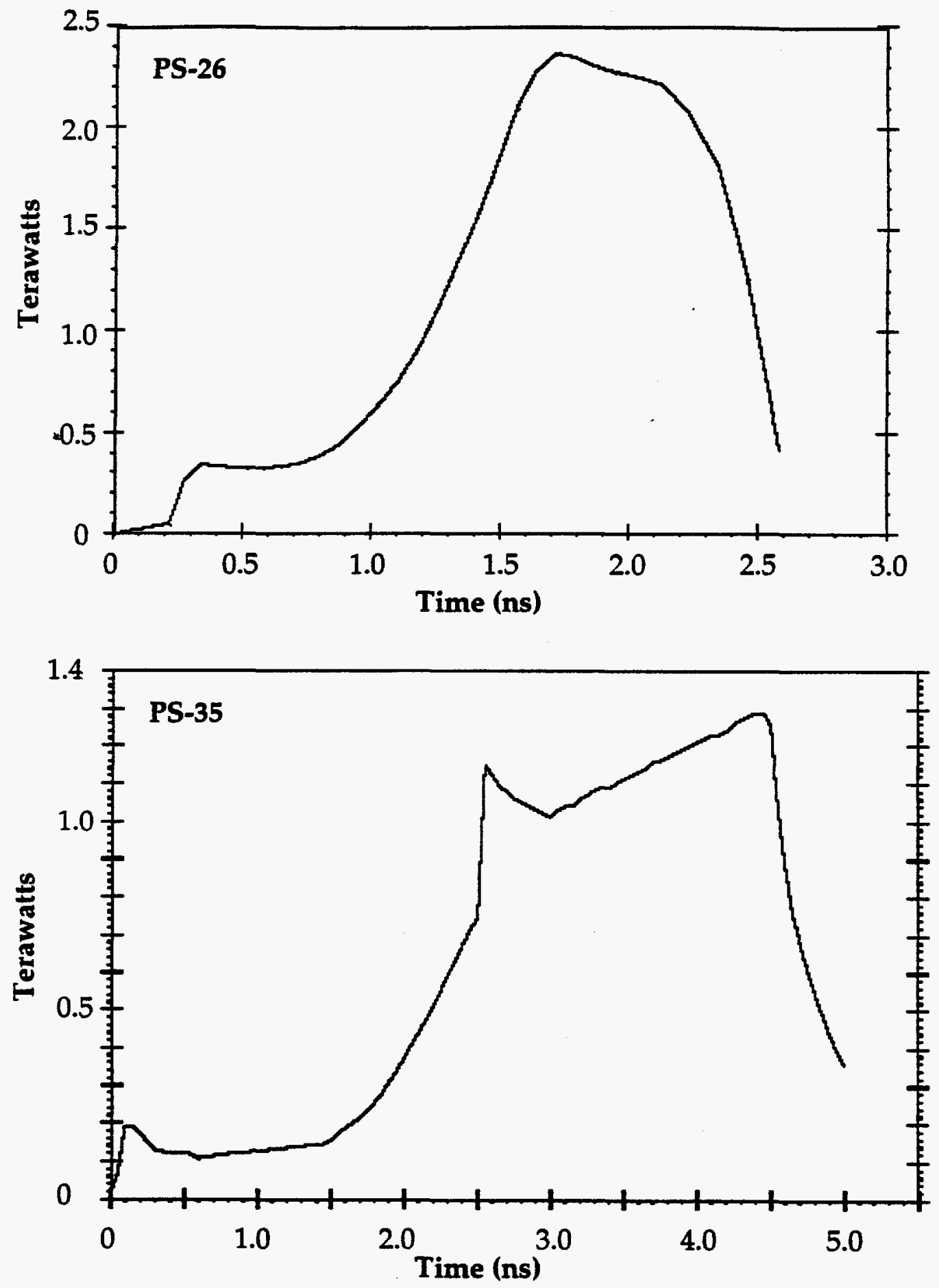
Figure 1-7: Packages That Were Shot

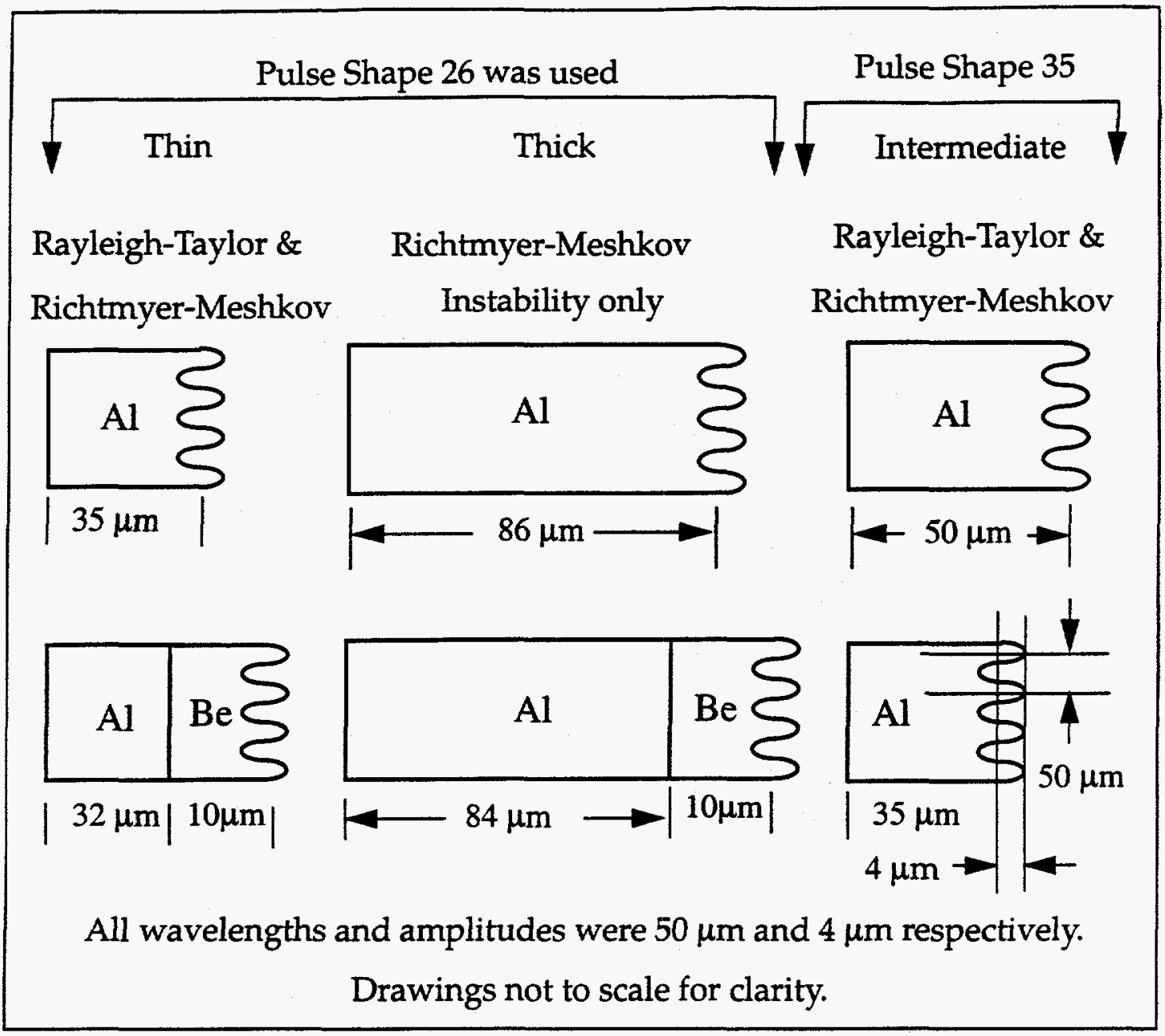

Both the thick and thin packages used a $2.2 \mathrm{~ns}$ long shaped pulse referred to as "PS-26," which produced a peak hohlraum temperature of $210 \mathrm{eV}$. A time dependent X-ray spectrum for PS-26 had previously been determined by calculation and experiment and was used in the feed-out calculations to drive the packages. The total energy of the spectrum could be increased or decreased in the calculation, but the spectrum as a function of time was fixed. The spectrum was non-Planckian and included contributions from the gold M-band emission. 
In between the weak and strong coupling extremes was the most interesting situation, a foil that moved from independent Rayleigh-Taylor and Richtmyer-Meshkov instabilities to coupled instabilities as it burned through. The intermediate situation was closest to the NIF capsules.

The intermediate packages, which were 50 and $35 \mu \mathrm{m}$ of aluminum, demonstrated a large amount of Rayleigh-Taylor growth. During the entire time the foils were moving from a decoupled to a coupled instability state, the Rayleigh-Taylor instability was causing the surface perturbations to grow. The intermediate case thus resulted in the greatest amount of Rayleigh-Taylor growth, and was easily designed by simply considering what factors would maximize the Rayleigh-Taylor growth. Package thicknesses were estimated by two competing factors. The package needed to be thick enough not to burn through before the pulse was over but as thin as possible in order for the Rayleigh-Taylor seed to reach the ablation front quickly. A long pulse was also needed to allow plenty of time for the instabilities to independently form, then to grow together as the foil burned through.

The pulse of choice for the intermediate packages was pulse shape 35, "PS-35," a 4.5 ns long pulse with a peak hohlraum temperature of about $160 \mathrm{eV}$. The PS-35 source used in the calculations was a time dependent Plankian obtained from experimental observation.

Beryllium was placed on the cold surface of some of the packages to study the effect a density jump would have on the feed-out. The density of beryl- 
lium is $1.85 \mathrm{~g} / \mathrm{cm}^{3}$ compared to aluminum's $2.7 \mathrm{~g} / \mathrm{cm}^{3}$. An experimental benefit in using beryllium was its transparency to the backlighter. The initial perturbation in the beryllium could be observed growing into the aluminum ablator from the cold surface. Originally, each composite package was intended to be massmatched to the pure aluminum package shown above it in the Figure 1-7. Massmatching would have created similar accelerations and instability growth rates, making the two easier to compare. Due to fabrication errors, this was not exactly the case.

Aluminum was selected as the ablator material for several reasons. Ablator material in ICF capsules is always composed of low $Z$ elements, usually with a $Z$ less than 6 . This is because atoms of a lower mass more efficiently convert the radiation into compressional energy. To better simulate the material of capsule ablators, it was desirable to use a low $Z$ material in the feed-out shots. Unfortunately, low $Z$ materials also have very low opacities and cannot effectively be observed with an X-ray camera. Aluminum was a good compromise as it is one of the lower $Z$ elements whose opacity is suitable for $X$-ray backlighting. It also has a well-characterized equation of state, is easy to work with, and is readily available.

The initial perturbation on all packages had a $50 \mu \mathrm{m}$ wavelength and 4 $\mu \mathrm{m}$ amplitude. The wavelength was selected to be approximately the same as package thickness, increasing the feed-out. The large initial amplitude introduced some nonlinear effects but was nevertheless desirable in order to insure that the 
perturbations were diagnostically observable.

Experimental parameters varied slightly on each shot, and several shots were needed to obtain a good time history for each package. Some of these variations, such as drive energy, were recorded. Others, such as package thickness variations, could not be accurately ascertained.

After the experiments were completed, better calculations were pursued than those used in the design. Recorded experimental parameters from the shots and better opacities were included in later computational runs. Mathematica was used to Fourier-analyze the results and compare the time histories of the first, second, and third harmonics to experimental data. Zone size and radiation bin size were reduced until convergence was achieved in perturbation growth and radiation temperature respectively.

In summary, feed-out is an important effect in ICF physics because it couples the larger internal perturbations with the long-lived, strong ablation surface instabilities. Feed-out is believed to be partially responsible for the superiority of beryllium capsules designs to plastic capsule designs. In order to better understand the nature of feed-out, an experimental and computational campaign was undertaken to study the effects of a density jump and variations in the level of coupling between the cold and hot surfaces. 


\section{Theory}

The physics of the feed-out packages may be understood with a system of coupled, nonlinear radiation-hydrodynamic equations, which will be discussed in this section. The radiation diffusion and Saha equations will be reviewed first, followed by a discussion on the equations of hydrodynamics. Special attention will be given to Rayleigh-Taylor and Richtmyer-Meshkov instability theories as aspects of the hydrodynamics.

\subsection{Radiation}

Radiation is an important aspect of the experiments presented in this paper. Radiation from the hohlraum ablates the feed-out foils and creates the shock wave which generates the Richtmyer-Meshkov instability. It preheats the packages and accounts for some of the heat transfer between different parts of the foils.

Radiation flow through the fluid is determined by the radiation transfer equation, which is a type of conservation equation. In Eulerian form, it may be written [Zel'dovich], as:

$$
\frac{1}{c}\left(\frac{\partial I_{v}}{\partial t}+c \hat{\Omega} \bullet \nabla I_{v}\right)=j_{v}\left(1+\frac{c^{2}}{2 h v^{3}} I_{v}\right)-\kappa_{v} I_{v},
$$

where $c$ is the speed of light, $h$ Planck's constant, and $\kappa_{v}$ and $j_{v}$ the absorption and emission coefficients for frequency $v$ respectively. The spectral radiant intensity is 
$I_{v} I_{v}(\bar{r}, \bar{\Omega}, t) d v d \bar{\Omega}$ is the radiant energy in the spectral interval $d v$, passing per unit time, through a unit area, with the direction of energy propagation contained within the element of solid angle $d \bar{\Omega}$ about the vector $\bar{\Omega}$ The area is located at point $\bar{r}$ and is perpendicular to $\bar{\Omega}$ The second term on the left-hand-side of Eq. (1) is the rate at which radiation is leaving or entering the differential volume. Of the three terms on the right-hand-side, the first is spontaneous emission while the second is induced emission. Induced emission is emission caused by interaction of the radiation field with the material. The third is absorption.

Eq. (1) is a partial differential equation for radiation intensity as a function of position, time, and direction and describes a nonequilibrium radiation field. The effort required to solve Eq. (1) may be significantly reduced by assuming local thermodynamic equilibrium (LTE) and the diffusion approximation. LTE implies that the temperature of a sufficiently extended and optically thick material varies little over the mean free path of the photons, so one may assume the local distribution is Planckian instead of having to calculate it. From the viewpoint of a given test location, photons coming from a position with a different temperature and Planckian spectrum would be absorbed well before reaching the test point.

Radiation fields in LTE may be modeled with the diffusion approximation. The necessary condition for the existence of LTE, small temperature gradients in an extended, optically thick medium, serves simultaneously as justification for the use of diffusion theory because it forces the radiation intensity 
into an almost isotropic state. If the radiation is anisotropic, then the diffusion approximation may not be used. Caution must be exercised in the application of diffusion to radiation, as the LTE assumption, isotropy, and therefore diffusion, may not be valid for all frequencies. For example, the peak of the hohlraum radiation spectrum used to drive the feed-out packages is about $150-200 \mathrm{eV}$. For most of the plasma, LTE at this temperature is a good assumption, but not for the ablated material. In addition, there are high energy components to the spectrum in the $\mathrm{keV}$ range. These photons stream through the packages with e-folding distances on the order of the size of the package, breaking the LTE assumption. As they represent only a small portion of the total energy in the radiation field, diffusion may still be used.

Diffusion in a radiation field in LTE may be written as:

$$
\bar{S}=\int I_{v} \bar{\Omega}(d \bar{\Omega}) d v=-\left(\frac{16}{3} \sigma l T^{3}\right) \nabla T,
$$

stating that the flux of radiant energy of all frequencies, $\bar{S}$, is proportional to the gradient of the temperature, $T$. The symbol $l$ is the average mean free path for a Planckian distribution over all frequencies and is commonly termed the Rosseland mean free path. The total energy lost or gained per unit volume of fluid, per unit time due to radiation transport, $q$, is now simply $q=\nabla \bullet \bar{S}$. Thus, by assuming LTE and diffusion, the radiation transfer problem has been reduced to a problem very similar to heat transport. This approximation and is frequently known as the radiation heat conduction approximation [Duderstadt]. 
Some comments should be made in regard to the opacity of materials [Zel'dovich], $\mu$, which is the sum of the scattering and absorption coefficients usually designated by $\mathrm{K}^{\prime} \mathrm{s}$. Opacity is a strong function of the temperature of a material, because the opacity for the three forms of absorption, bound-bound, boundfree, and free-free, are so different. Bound-bound transitions correspond to the electron changing orbitals in the atom and have extremely large cross sections for very specific energies. Bound-free transitions can be from eight to eleven orders of magnitude lower in cross section than bound-bound, but represent a continuum of energies, unlike the bound-bound. A bound-free transition occurs when an electron is ejected from the atom. A free electron can only absorb energy from a photon if it is passing very close to an ion. If an ion is not in the vicinity, the electron can only serve as a scattering center for the photon. This type of absorption is referred to as free-free or inverse bremsstrahlung and has a cross section proportional to the square of the ion density. Of the three types of transitions, free-free tends to have the lowest cross section.

As temperature increases, the ionization increases, and so the opacity decreases. The density of ion states is thus very important for good opacities and is usually calculated with the Saha equation,

$$
\frac{n_{m+1}}{n_{m}}=\frac{2}{n_{e}} \frac{u_{m+1}}{u_{m}}\left(\frac{2 \pi m_{e} k T}{h^{2}}\right)^{3 / 2} \quad u_{m}=\sum_{i=1}^{m} e^{-E_{i} /(k T)} .
$$

The subscript $m$ denotes the ionization state; $n$ is the density of a particular state, $n_{e}$ is the density of electrons, $k T$ is temperature in units of energy, and $E_{i}$ is the 
excitation energy of the ion in the $i$ th state; $u$ is the partition function. The Saha equation is valid for local thermodynamic equilibrium.

From the above discussion, one can see the ablated material from the feed-out packages will be at a high temperature and have a low opacity and will be transparent to the hohlraum radiation. The ablation front will be composed of atoms in the process of being ionized and heated, and will absorb the bulk of the radiation from the hohlraum. Material behind the ablation front will have the highest opacity, and be the coldest. Only photons in the keV range will affect this region. Because of their long mean free path, the $\mathrm{keV}$ photons stream through the ablation front, depositing energy deep in the package. This results in expansion and preheat of the material before shock arrival, and may partially stabilize the Richtmyer-Meshkov instability by creating a density gradient.

\subsection{Hydrodynamics}

The physics of the feed-out experiments is dominated by hydrodynamics. The Rayleigh-Taylor and Richtmyer-Meshkov instabilities, which are the focus of these experiments, are hydrodynamic effects. The hydrodynamics controls the evolution of the perturbations, the density, velocity, pressure, and many other important fluid quantities.

The equations governing hydrodynamics are the equation of continuity, equation of motion, and the energy equation. The equation of continuity is derived by writing a mass balance over a volume of the fluid, and allowing the 
volume to approach zero. Likewise, the equations of motion and energy are derived by writing balances over momentum and energy. A detailed derivation and description of these equations may be found in the text by Bird, Stewart and Lightfoot [Bird].

Some comments should be made as to why fluid theory is acceptable for the highly ionized gases that compose ICF packages. Both electromagnetic and hydrodynamic forces are present in these gases. There are two conditions necessary for the hydrodynamic forces to dominate. First, the ratio of the electromagnetic potential energy to the kinetic energy of a particle must be small. The potential energy comes from a test particle's interaction with other charged particles in the gas. As the particle moves through the fluid, a large kinetic energy and small electromagnetic potential energy results in small changes in velocity and momentum as it passes other charged particles. This effect is similar to what one would expect in a nonionized gas. The ratio of these two energies is easily calculated, as it is proportional to the inverse of the number of particles in a Debye sphere [Krall]. The second condition is that the frequency of collisions must be much greater than the plasma frequency. High collision frequency ensures that before the fields can effectively move a test particle any distance, collisions are pushing it in the direction of the flow.

There are two formulations of hydrodynamics. The Eulerian or conservative formulation is derived by writing the balance over a stationary volume element through which the fluid flows, and it represents the viewpoint of an 
observer in the laboratory frame. In the Lagrangian formulation, the volume is flowing with the fluid, and the equations predict changes that occur as one follows a path with the fluid motion. These two methods are equivalent.

Eqs. (3)-(5) listed below are in Eulerian form. The variables are, $\rho$ density, $v$ velocity, $t$ time, $p$ pressure, $g$ gravity, $U$ internal energy, $q$ heat flux, and $\zeta$ is the shear force per unit area due to viscosity. Velocity, heat flux and gravity are vectors, while $\zeta$ is a tensor. The equation of continuity is

$$
\frac{\partial \rho}{\partial t}=\quad-(\nabla \cdot \rho \bar{v}) .
$$

Rate of mass accumulation in differential volume at some point in the fluid.
Rate of mass flux in or out of differential volume.

The equation of motion is

$$
\frac{\partial}{\partial t} \rho \bar{v}=
$$

$$
\begin{aligned}
& \text { Rate of increase of } \\
& \text { momentum per } \\
& \text { unit volume. }
\end{aligned}
$$$$
\text { Rate of momentum }
$$$$
\text { gain by convection }
$$

Pressure force per unit volume. on element per

$$
-\nabla \cdot \underline{\zeta}
$$

Rate of momentum gain by viscous transfer per unit volume.

$$
+\rho \bar{g} \text {. }
$$


The equation of energy is given by

$$
\frac{\partial}{\partial t}\left(U+\frac{v^{2}}{2}\right)=\quad-\left(\nabla \cdot \rho \bar{v}\left(U+\frac{v^{2}}{2}\right)\right)
$$

Rate of gain of Rate of energy input Rate of energy input internal and kinetic per unit volume by per unit volume by energy per unit volume. convection. heat convection.

$$
+\rho(\bar{v} \cdot \bar{g})
$$

$$
-(\nabla \cdot[\underline{\zeta} \bullet \bar{v}])
$$

Rate of work done on Rate of work done on Rate of work done on volume by gravity. fluid per unit volume fluid per unit volume by pressure forces. by viscous forces.

The Lagrangian formulation is written using a derivative following the fluid motion. This is called a substantial derivative, and is given by

$$
\frac{D \psi}{D t}=\frac{\partial \psi}{\partial t}+\bar{v} \cdot \nabla \psi=\frac{\partial \psi}{\partial t}+v_{x} \frac{\partial \psi}{\partial x}+v_{y} \frac{\partial \psi}{\partial y}+v_{z} \frac{\partial \psi}{\partial z}
$$

Applying this derivative to Eqs. (3)-(5) and using the continuity equation to simplify the results, the Lagrangian form is found to be

$$
\begin{gathered}
\frac{D \rho}{D t}=-\rho(\nabla \bullet \bar{v}) \\
\rho \frac{D \bar{v}}{D t}=-\nabla p-[\nabla \bullet \underline{\zeta}]+\rho \bar{g} \\
\rho \frac{D}{D t}\left(U+\frac{v^{2}}{2}\right)=-\nabla \bullet \bar{q}+\rho(\bar{v} \bullet \bar{g})-(\nabla \bullet \rho \bar{v})-(\nabla \bullet[\underline{\zeta} \bullet \bar{v}]) .
\end{gathered}
$$

Notice the equation of motion in Eqs. (6) is now in the form of Newton's second law, mass $x$ acceleration $=$ force. 
There are three equations, not counting vectors, and four unknowns in the system of equations above, with the unknowns being $\bar{v}, U, \rho$, and $p$. The system needs to be closed with an equation of state, which is an equation relating one thermodynamic property to two others. In this case, there needs to be a relationship between $U, \rho$, and $p$. For an ideal gas, one may use $p=R \rho U$ where $R$ is a constant of proportionality. Unfortunately, this is an overly simplified expression for ICF plasmas, which normally require the use of a tabulated equation of state from detailed calculations.

The hydrodynamic theories presented in the following sections often entail many assumptions in order to obtain simple, closed form solutions. The assumptions severely limit the practicality of the results, but they also allow for clearly understandable theories, providing great insight into the fundamental workings of the physics. Computational solutions are used to study the complete problems of radiation-hydrodynamics with fewer assumptions.

The following four sections focus on the Rayleigh-Taylor instability. A general overview of the Rayleigh-Taylor instability will be provided in section 2.2.1, then a derivation of the classical linear growth rate in section 2.2.2. A discussion on the effects of a density gradient and ablation will ensue. Section 2.2 .3 is a review of mode coupling, while 2.2.4 discusses the effects of stratified fluids on the Rayleigh-Taylor instability. The Richtmyer-Meshkov instability will be presented in section 2.2 .5 with a short discussion of mode coupling. The RichtmyerMeshkov instability in stratified fluids is presented in section 2.2.6. Acoustic 
modes, which are a result of the Richtmyer-Meshkov instability, will then be discussed in section 2.2.7. Section 2.2 .8 presents a simple theory for feed-out from differential acceleration.

\subsubsection{Introduction to the Rayleigh-Taylor Instability}

The Rayleigh-Taylor instability occurs when the density and pressure gradients in a fluid have opposite signs, in other words, whenever a heavy fluid is accelerated by a lighter fluid. In this context, heavy and light refers to higher and lower density fluids respectively. A good example is when one turns a container of oil and water upside-down. The oil, which is a lighter fluid, is then supporting the water against acceleration from gravity. If the interface is perfectly flat, the system is stable, but if there are any perturbations on the interface, stability is lost and the perturbations begin to grow. The water moves to the bottom and the oil to the top. Entropy is thus increased by converting a state of ordered energy storage, potential energy of a top heavy fluid, to a state of disordered energy storage, turbulence and heat generated through viscosity.

Precisely how perturbations grow has been of intense interest to ICF researchers. Perturbation growth determines how capsule symmetry may be lost when different fluids begin to mix. The evolution of a single sinusoidal perturbation is particularly instructive in understanding the Rayleigh-Taylor instability, because any initial perturbation on the interface may be decomposed into a Fourier spectrum. The time history of a single mode is displayed in Figure 2-1. With 
Figure 2-1: Time History of Rayleigh-Taylor Instability

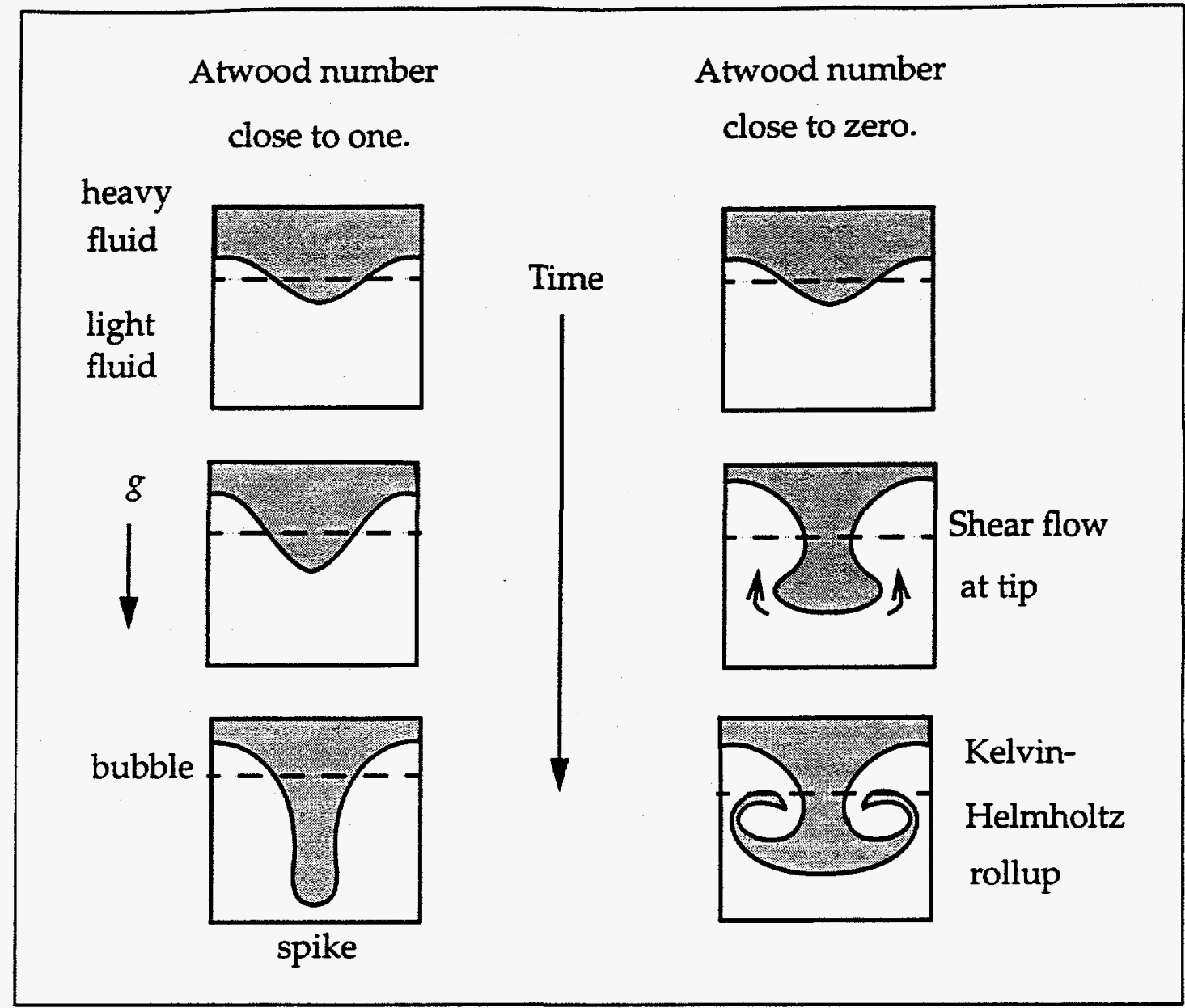

weak shocks and amplitudes less than a tenth the wavelength, the perturbation will grow exponentially early in time. Exponential growth may be derived by linearizing the hydrodynamic equations and is thus the linear phase of the instability. The time history of the perturbation amplitude, $\eta$, in this stage is given by

$$
\frac{\eta(t)}{\eta_{0}}=e^{\gamma t} \quad \gamma=\sqrt{g k A} \quad k=\frac{2 \pi}{\lambda},
$$

where $\eta_{0}$ is the initial amplitude, $k$ is the wavenumber, $\lambda$ is the wavelength, and $\gamma$ is the growth rate. The Atwood number, $A$, is defined as: 


$$
A=\frac{\rho_{H}-\rho_{L}}{\rho_{H}+\rho_{L}}
$$

where $\rho_{H}$ and $\rho_{L}$ are the densities of the heavy and light fluids respectively. The acceleration $g$ is considered positive if it is directed from the heavy to light fluid. In that case, there is exponential growth of the perturbation. If the acceleration is directed from the light to the heavy fluid, then it is considered negative, meaning $\gamma$ is imaginary. This results in stable oscillations, such as water waves.

When the amplitude is somewhere between a tenth to a whole wavelength, nonlinear effects become dominant and the growth slows down. At this point, the nature of the growth depends strongly on the Atwood number. For $A \cong 1$ the dominant effect is volume conservation of the high density fluid, with the constraint that the gravitational potential energy is minimized [Haan June 1991]. The light fluid flows upward into the heavy fluid in rounded shapes called bubbles, while the heavier fluid falls into the lighter fluid in long narrow spikes. The potential energy of the system is decreased by narrowing the spikes, with the tips moving further down.

The spike tips eventually reach free fall, while the bubbles rise at a constant velocity proportional to [Hoffman 1994]

$$
v_{B}=\alpha \sqrt{\frac{2 A}{1+A} g \lambda}
$$

where $\alpha$ is a constant. This expression is the consequence of a balance between the buoyancy and drag forces acting on the bubble. 
For $A \cong 0$ as the spikes are being formed, they become altered by the Kelvin-Helmholtz rollup [Haan June 1991]. This is a shear instability that occurs at the interface of two fluids with different tangential velocities, see Figure 2-1. The linear growth rate for the Kelvin-Helmholtz instability is given by [Haan June 1991]

$$
\gamma=k^{2} v_{R}^{2} \frac{\rho_{1} \rho_{2}}{\left(\rho_{1}+\rho_{2}\right)^{2}},
$$

where $\rho_{1}$ and $\rho_{2}$ are densities of the two fluids and $v_{R}$ is their relative velocity. As a spike pushes into the lighter fluid, there is a velocity differential between the tip of the spike and bottom of the bubble. The density term in $\gamma$ for the Kelvin-Helmholtz instability is high, creating a fluid instability at the spike tips and retarding their growth. The result for small Atwood numbers is a symmetric mushroom between the light and heavy fluids. For intermediate Atwood numbers, one finds a spike with a mushroom on top. The feed-out packages have an Atwood number close to one, so the Kelvin-Helmholtz instability is not a concern.

Modeling of the Rayleigh-Taylor instability through its entire evolution requires different kinds of theoretical approaches. While $\eta<\lambda / 10$, the linear theory is adequate. For the weakly nonlinear regime, potential flow models may be used, which assume the velocity may be described as a harmonic potential. Haan developed such a model, which will be described later [Haan August 1991]. It is valid only to second order, representing growth of the second harmonics. For the strongly nonlinear case with multiple interacting modes, a model was proposed 
by Ofer [Ofer], improving slightly on one previously proposed by Haan [Haan June 1991, Hoffman 1994]. Ofer and his colleagues suggested using Haan's second order potential flow model up to the point where a mode begins to saturate, then switching over to a linear growth prescription more representative of saturation. The model is part analytical and part empirical. For very late times the Rayleigh-Taylor instability in the spike and bubbles phase, analytic theory may be used to predict very limited things such as the terminal bubble velocity. More commonly this regime is analyzed through computations and experiment and described by empirical formulas.

\subsubsection{Single Mode Rayleigh-Taylor Growth}

Following Hoffman [Hoffman 1994], the Rayleigh-Taylor instability growth of a single mode will be derived for a classical instability. The expression for the growth rate will then be amended by including terms to account for ablative stabilization, which is present in the feed-out experiments. The starting point is Eqs. (3) and (4), with the viscosity term removed from the momentum equation. By substitution of Eq. (3) into Eq. (4), one may obtain a simpler form of the momentum equation. The two equations together then are

$$
\begin{gathered}
\frac{\partial \rho}{\partial t}+\nabla \cdot(\rho \bar{v})=0 \\
\rho \frac{\partial}{\partial t}(\bar{v})+\rho(\bar{v} \bullet \nabla) \bar{v}=-\nabla p+\rho \bar{g} .
\end{gathered}
$$

A small perturbation is introduced into the system by replacing each hydrody- 
namic property in Eqs. (7) with two quantities, Eq. (8)

$$
\begin{aligned}
& \rho=\rho_{0}+\rho_{\pi} \\
& \bar{v}=\bar{v}_{0}+\bar{v}_{\pi} \\
& p=p_{0}+p_{\pi}
\end{aligned}
$$

The variables with the " 0 " subscripts represent equilibrium and are the "zerothorder" aspect of the fluid motion. They satisfy Eqs. (7) by themselves, so one may write

$$
\begin{gathered}
\frac{\partial \rho_{0}}{\partial t}+\nabla \bullet\left(\rho_{0} \bar{v}_{0}\right)=0 \\
\partial \bar{v}_{0}+\rho\left(\bar{v}_{0} \cdot \nabla\right) \bar{v}_{0}=-\nabla p_{0}+\rho_{0} \bar{g}
\end{gathered}
$$

The quantities with the subscript " $\pi$ " in Eqs. (8) represent the perturbation being placed on the equilibrium properties and are very small. They are sometimes referred to as the "first-order" quantities.

After substituting Eqs. (8) into Eqs. (7), Eqs. (9) are subtracted, and the result is linearized. The process of linearization involves the elimination of any products of perturbed quantities, as these will be much smaller than terms that are linear in the first order terms. The linearized equations are

$$
\begin{aligned}
& \frac{\partial \rho_{\pi}}{\partial t}+\nabla \cdot\left(\rho_{\pi} \bar{v}_{0}+\rho_{0} \bar{v}_{\pi}\right)=0 \\
& \rho_{\pi} \frac{\partial \bar{v}_{0}}{\partial t}+\rho_{0} \frac{\partial \bar{v}_{\pi}}{\partial t}+\rho\left(\bar{v}_{\pi} \bullet \nabla \bar{v}_{0}+\bar{v}_{0} \bullet \nabla \bar{v}_{\pi}\right)+\rho_{\pi}\left(\bar{v}_{0} \bullet \nabla \bar{v}_{0}\right)=-\nabla p+\rho_{\pi} \bar{g}
\end{aligned}
$$

Now an initial condition is applied to Eqs. (10). For the Rayleigh-Taylor instability problem, one assumes the two fluids are initially at rest, implying, 
$\bar{v}_{0}=0$. In addition, one can make a simplifying assumption that the flow is incompressible. Incompressibility is valid if accelerations in the flow are not strong enough to significantly change the density, and the fluid moves without either expanding or compressing. To apply this assumption, the divergence of the perturbed velocity is set to zero

$$
\nabla \bullet \bar{v}_{\pi}=0
$$

Substituting the initial velocity and incompressibility into Eqs. (10), one obtains

$$
\begin{gathered}
\frac{\partial \rho_{\pi}}{\partial t}+\bar{v}_{\pi} \cdot \nabla \rho_{0}=0 \\
\rho_{0} \frac{\partial \bar{v}_{\pi}}{\partial t}=-\nabla p_{\pi}+\rho \bar{g}
\end{gathered}
$$

For this derivation, it is assumed that gravity only acts in one direction, which is from the heavy to the light fluid, and perpendicular to the interface. Let this direction be $\bar{z}$, giving

$$
g_{x}=g_{y}=0
$$

In addition, $\rho_{0}$ is uniform throughout each of the fluids, with the exception of a discontinuity occurring at the interface. Thus, one may write

$$
\frac{\partial \rho_{0}}{\partial x}=\frac{\partial \rho_{0}}{\partial y}=0
$$

Because it is nonzero at the interface, the derivative of $\rho_{0}$ with respect to $z$ must remain in the equations. Eqs. (13) and (14) are now substituted into Eqs. (12), with the result in component form below. Eq. (11) is also included because of 
its usefulness.

$$
\begin{array}{rlrl}
\rho_{0} \frac{\partial v t}{\partial x} & =\frac{\partial p_{\pi}}{\partial x} & & \frac{\partial \rho_{\pi}}{\partial t}+v_{\pi z} \frac{\partial \rho_{0}}{\partial z}=0 \\
\rho_{0} \frac{\partial v_{\pi y}}{\partial t} & =\frac{\partial p_{\pi}}{\partial y} & & \frac{\partial v_{\pi x}}{\partial x}+\frac{\partial v_{\pi y}}{\partial y}+\frac{\partial v_{\pi z}}{\partial z}=0 . \\
\rho_{0} \frac{\partial v_{\pi z}}{\partial t}=\frac{\partial p_{\pi}}{\partial z}-\rho_{\pi \delta} g &
\end{array}
$$

Eqs. (15) are solved by Fourier transforming, which converts the derivatives into products. Only transformations with respect to $x$ and $y_{p}$ are made, as the $z$ direction does not share the same symmetry as the other two directions. The following Fourier transform pairs are defined:

$$
\begin{aligned}
V_{\pi x}\left(k_{x}, k_{y}, z, t\right) \leftrightarrow v_{\pi x}(x, y, z, t) & P_{\pi}\left(k_{x}, k_{y}, z, t\right) \leftrightarrow p_{\pi}(x, y, z, t) \\
V_{\pi y}\left(k_{x}, k_{y}, z, t\right) \leftrightarrow v_{\pi y}(x, y, z, t) & R_{\pi}\left(k_{x}, k_{y}, z, t\right) \leftrightarrow \rho_{\pi}(x, y, z, t) \\
V_{\pi z}\left(k_{x}, k_{y}, z, t\right) \leftrightarrow v_{\pi z}(x, y, z, t) &
\end{aligned}
$$

The zeroth-order quantities are not functions of $x$ and $y$, which simplifies the Fourier transforms. $k_{x}$ and $k_{y}$ are the $x$ and $y$ components of the wavevector, $\bar{k}$, with a magnitude of $k=\sqrt{k_{x}^{2}+k_{y}^{2}}$, which is called the wavenumber. The wavelength that corresponds to a particular wavenumber is given by, $\lambda=(2 \pi) / k$. In solving Eqs. (15), one may assume the time dependence of the solution is proportional to $e^{i \gamma t}$, which is a standard assumption for finding solutions with the Fourier transform. After performing the transforms and substituting the time dependence, Eqs. (15) become 


$$
\begin{array}{ll}
\gamma \rho_{0} V_{\pi x}=-i k_{x} P_{\pi x} & \gamma R_{\pi}+V_{\pi z} \frac{\partial \rho_{0}}{\partial z}=0 \\
\gamma \rho_{0} V_{\pi y}=-i k_{y} P_{\pi y} & i k_{x} V_{\pi x}+i k_{y} V_{\pi y}+\frac{\partial V_{\pi z}}{\partial z}=0 . \\
\gamma \rho_{0} V_{\pi z}=-\frac{\partial P_{\pi}}{\partial z}-R_{\pi} g &
\end{array}
$$

After some algebraic manipulations of Eqs. (16) and with the help of the definition of the wavenumber, one may write

$$
\frac{\partial}{\partial z}\left(\rho_{0} \frac{\partial V}{\partial z}\right)=k^{2} \rho_{0} V_{\pi z}\left(1-\frac{g}{\gamma^{2} \rho_{0}} \frac{\partial \rho_{0}}{\partial z}\right)
$$

Eq. (17) is an eigenvalue problem, meaning it only has solutions for a specific pair of $\gamma$ and $V_{\pi z}$, once $k, g$, and $\rho_{0}(z)$ have been defined; $\gamma$ is the eigenvalue, while $V_{\pi z}$ is an eigenfunction.

Away from the interface, $\rho_{0}$ is constant, so its derivatives are zero. One may thus cancel it from Eq. (17) and remove the derivatives. This leaves

$$
\frac{\partial^{2} V_{\pi z}}{\partial z^{2}}=k^{2} V_{\pi z}
$$

which has the general solution,

$$
V_{\pi z}=A\left(k_{x}, k_{y}, t\right) e^{k z}+B\left(k_{x}, k_{y}, t\right) e^{-k z}
$$

The vertical velocity should vanish at infinity, implying $B=0$ for $z<0$ and $A=0$ for $z>0$. The two solutions should match at the interface, where at time zero there is some initial condition, $X\left(k_{x}, k_{y}\right)=V_{\pi z}\left(k_{x}, k_{y}, z=0, t=0\right)$. The solu- 
tion should also contain an exponential time dependence, as this was the basis for the derivation of Eqs. (16), resulting in the solution

$$
V_{\pi z}=\left\{\begin{array}{l}
X\left(k_{x}, k_{y}\right) e^{k z} e^{\gamma t}, z<0 \\
X\left(k_{x}, k_{y}\right) e^{-k z} e^{\gamma t}, z>0
\end{array}\right\} .
$$

To calculate $\gamma$, an additional boundary condition is needed. By integrating Eq. (17) over the interface along an infinitesimally small element of the $z$ axis, a jump condition may be obtained

$$
\begin{array}{r}
\lim _{\varepsilon \rightarrow 0} \int_{-\varepsilon}^{+\varepsilon}\left(\frac{\partial}{\partial z}\left(\rho_{0 \frac{\partial}{\partial z}} \pi z\right)-k^{2} \rho_{0} V_{\pi z}\left(1-\frac{g}{\gamma^{2} \rho_{0}} \frac{\partial \rho_{0}}{\partial z}\right)\right) d z=0 \\
-k W\left(\rho_{H}+\rho_{L}\right)+\frac{k^{2} g}{\gamma^{2}} W\left(\rho_{H}-\rho_{L}\right)=0
\end{array}
$$

Solving for $\gamma$,

$$
\gamma^{2}=k g \frac{\left(\rho_{H}-\rho_{L}\right)}{\left(\rho_{H}+\rho_{L}\right)}=k g A .
$$

Eq. (19) shows the origin of the Atwood number, $A$. Notice there is a positive and negative component of the square root, yielding growing and decaying modes.

At least two other factors add stability to radiation-ablated plasmas that are not accounted for in Eq. (19), a density gradient and the ablation process itself. In the feed-out experiments, ablation partially stabilizes the instabilities on the hot surface, while a density gradient partially stabilizes instabilities on both surfaces. Density gradient stabilization will be presented first. 
Consider a more realistic ICF situation including radiation from either a hohlraum or a laser. As radiation ablates material from a solid foil, the ablated material streams away into vacuum, and a flat density gradient is formed. The growth of a perturbation on this gradient is an eigenvalue problem like the growth on a sharp interface, with the specific eigenvalue and eigenfunction depending on the density profile. For an exponential density profile, the eigenfunction is exponential, as in the sharp interface problem. The eigenvalue cannot be written down in closed form, although one may approximate it.

A back of the envelope estimate is possible by deriving an effective Atwood number [Haan June 1991]. In the sharp interface situation, the perturbation falls off away from the interface as $e^{-k|z|}$, thus sampling effective densities at $z= \pm 1 / k$. Assuming the density varies as $e^{z / L}$, where $L$ is some scale length, then the effective densities of the perturbation samples are

$$
\rho_{H}=\rho_{0} e^{1 /(k L)} \quad \rho_{L}=\rho_{0} e^{-1 /(k L)} .
$$

Giving an effective Atwood number and growth rate of

$$
\begin{gathered}
A=\frac{\rho_{H}-\rho_{L}}{\rho_{H}+\rho_{L}}=\tanh \left(\frac{1}{k L}\right) \\
\gamma=\sqrt{g k \tanh \left(\frac{1}{k L}\right)} .
\end{gathered}
$$

For $k L » 1$, the perturbation wavelength is much smaller than the density scale length, resulting in an effective Atwood number close to zero. This represents the maximum stabilizing effect of the gradient, with the growth rate 
approaching $\gamma \rightarrow \sqrt{g / L}$. For $k L \ll 1$, the perturbation is much bigger than the density scale length. The effective Atwood number approaches one, with the growth rate becoming, $\gamma \rightarrow \sqrt{g k}$. Stabilization of the interface thus occurs for modes with a wavenumber greater than about $k>1 / L$. A more rigorously derived and frequently encountered solution to this problem is [Haan June 1991, Hoffman 1994]

$$
\gamma=\sqrt{\frac{g k A}{1+k L}}
$$

which is not significantly different from Eq. (20).

Another important stabilizing effect in radiation ablated fluids comes from the ablation process itself. Think of the interface from the accelerating reference frame in which the heavy fluid is stationary, see Figure 2-2. Assume the perturbation in the heavy fluid has the classical fall off and growth rate, $e^{-k z}$ and $e^{\gamma t}$, respectively. Consider the situation after a time, $\Delta t$ from time zero. The perturbation has grown by $e^{\gamma \Delta t}$, but the interface has moved much deeper into the heavy fluid from ablation. The portion of the interface "shaved off" by ablation is given by $v_{A} \Delta t$, where $v_{A}$ is the interface velocity in the heavy fluid. This is given by

$$
\left.v_{A}=\frac{1}{\rho_{H}} \frac{\partial m}{\partial t}=\quad \text { (mass ablation rate per } \mathrm{cm}^{2}\right) / \rho_{\mathrm{H}}
$$

This movement of the interface results in a decrease in the perturbation amplitude of $k v_{A}$. The net growth is then [Haan June 1991] 


$$
\gamma=\gamma_{c}-\beta k v_{A}
$$

where $\beta$ is a constant that must be determined by experiment and/or computation. The $c$ subscript on $\gamma$ refers to the classical growth rate. Eq. (21) is a simple formula that has not been rigorously derived, but is used extensively in ICF research and first introduced by Takabe [Takabe]. As with the density gradient problem, there is no rigorous closed form solution to the eigenvalue problem of an ablated interface.

Consider the implications of the ablation process. If $\gamma_{c} \leq \beta k v_{A}$, then the total growth rate is zero or negative, which occurs at the threshold wavelength of $\lambda=\left(2 \pi \beta^{2} v_{A}^{2}\right) /(g A)$. Wavelengths shorter than this value will not grow Figure 2-2: Ablative Stabilization

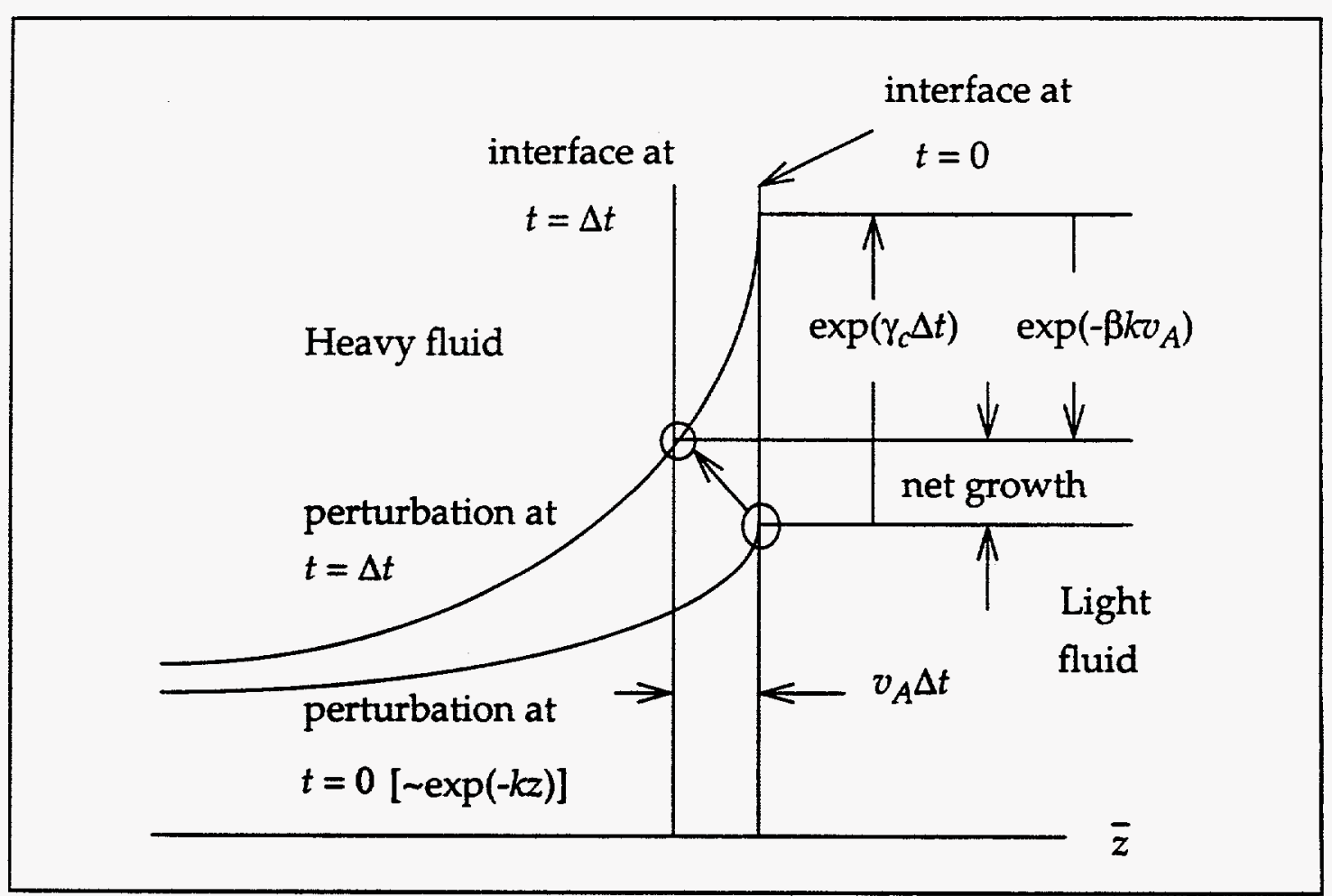


due to the stabilizing influence of the ablation. As the size of the wavelength is increased above this value, ablation will play less and less of an effect. Ablation, like the density gradient, serves to stabilize the shorter wavelengths.

One may combine the stabilizing effects of burn-off and a density gradient into one formula [Haan June 1991, Hoffman 1994]

$$
\gamma=\sqrt{\frac{g k A}{1+k L}}-\beta k v_{A} .
$$

Eq. (22) is an expression for incompressible fluids, while laser ablated plasmas are compressible. Compressibility effects produce a small net change in growth rate, under $20 \%$, from the incompressible state depending on the size of the wavelength [Haan June 1991].

Some care should be taken with the application of Eq. (22). The linearized perturbation equations, Eqs. (10), assume the perturbed quantities to be small in relation to the equilibrium quantities. If this is not true, Eq. (22) is no longer valid. Eq. (22) is only applicable for small perturbations with an amplitude less than $10 \%$ of the wavelength, and early time periods in the growth.

A great deal of effort has been put into refining Eq. (22). However, there is some question as to the utility of continuing to refine the expression. Mathematical solutions require mathematical assumptions as to the nature of the density gradient, ablation rate, and many other properties of the system. The actual experimental values of these properties may not be well known. Eq. (22) is only as accurate as the assumptions which were used to create it. Refining the expression 
to a mathematical level of accuracy beyond the uncertainties in the assumptions is futile.

\subsubsection{Multiple Mode Rayleigh-Taylor Growth}

At the onset of nonlinearity, the single mode begins to couple with itself, producing higher order harmonics which in turn couple with each other. The result of the addition of these higher order modes is the bubble and spike formation. The theory of mode coupling is important for a proper understanding of the Rayleigh-Taylor instability and the feed-out experiments. Both higher harmonics and bubble and spike formation were experimentally observed in some of the packages. One would expect that the growth of higher harmonics in the feedout experiments would be slightly less than that predicted by the following theories. Both ablation and density gradients stabilize higher order modes more effectively than lower order ones, and both stabilization processes are present in the experiments.

Three models of the nonlinear Rayleigh-Taylor instability will be discussed, each corresponding to a different stage in its evolution. The first is Haan's weakly nonlinear mode coupling model which describes fluid behavior at the beginning of the nonlinear regime with only second harmonics present [Haan June 1991, August 1991]. A second Haan model assumes a later time, with many harmonics present and a large number of saturated modes. The last model by Youngs is applicable during late times the nonlinearity, when the interface is so 
mixed that the concept of Fourier modes is no longer valid [Youngs].

In the early stages of the nonlinear regime, fundamental modes begin to couple with themselves creating second harmonics. Self-coupling is clearly seen in Haan's weakly nonlinear theory. The theory is a potential flow model and was derived assuming a plane interface between inviscid, incompressible fluids, with surface tension, $T$. The system is initially at rest in a gravitational field with the interface located at $z=\eta(\bar{x}, t)$, where $\bar{x}=(x, y)$. Periodic boundary conditions apply at the edges of a box of length $L$ in the plane of the interface, and the fluid is assumed stationary as $z \rightarrow \pm \infty$. The interface may be decomposed into modes of a Fourier series. Their coefficients are given by

$$
\eta_{\bar{k}}(t)=\frac{1}{L^{2}} \int \eta(\bar{x}, t) e^{-i \bar{k} \cdot \bar{x}} d \bar{x},
$$

where $\bar{k}=\left(k_{x}, k_{y}\right)$.

Let $\phi_{H}$ and $\phi_{L}$ be the velocity potentials in the heavy and light fluids respectively. These potentials may likewise be Fourier expanded in a consistent fashion with the boundary conditions

$$
\begin{aligned}
& \phi_{H}(\bar{x}, z, t)=\sum_{\bar{k}} \phi_{H \bar{k}}(t) e^{-k z} e^{-i \bar{k} \cdot \bar{x}} \\
& \phi_{L}(\bar{x}, z, t)=\sum_{\bar{k}} \phi_{L \bar{k}}(t) e^{-k z} e^{-i \bar{k} \cdot \bar{x}} .
\end{aligned}
$$

The pressure of each fluid is given by Bernouli's equation and is 


$$
p=\rho\left(\frac{\partial \phi}{\partial t}-\frac{v^{2}}{2}-g z\right)
$$

There are three boundary conditions at the interface. First, the pressure step across the interface is determined by the surface tension and the curvature

$$
\frac{\partial \eta}{\partial t}=\left.\frac{\partial \eta \partial \phi}{\partial x \partial x}\right|_{\eta}+\left.\frac{\partial \eta \partial \phi}{\partial y \partial y}\right|_{\eta}-\left.\frac{\partial \phi}{\partial z}\right|_{\eta}
$$

This is actually two equations, $\phi$ may either be $\phi_{H}$ or $\phi_{L}$. The two equations must agree, which is the second boundary condition. The third condition states that the pressure step across the interface must be a function of the surface tension and curvature of the interface

$$
p_{H}-p_{L}=T\left(\frac{d^{2} \eta}{d x^{2}}+\frac{d^{2} \eta}{d y^{2}}\right)+\text { HOT }
$$

HOT refers to higher order terms that are neglected in this analysis.

Haan substitutes Eqs. (23) - (25) into the boundary conditions Eqs. (26) and (27). The boundary conditions are applied at $z=\eta(x, t)$ instead of $z=0$, because the interface is located at $z=\eta(x, t)$. For example, the term $\partial \phi / \partial z$ would be expanded as

$$
\left.\frac{\partial \phi}{\partial z}\right|_{\eta}=\left.\frac{\partial \phi}{\partial z}\right|_{\eta=0}+\eta\left|\frac{d^{2} \phi}{d z^{2}}\right|_{\eta=0}+H O T .
$$

Combining the boundary conditions, Haan arrived at a differential equation for the time evolution of the interface 


$$
\ddot{\eta_{\bar{k}}}=\gamma^{2}(k) \eta_{k}+A k \sum_{\bar{k}_{2}}\left[\ddot{\eta}_{\bar{k}_{2}} \eta_{\bar{k}_{3}}\left(1-\hat{k}_{2} \bullet \hat{k}\right)+\dot{\eta}_{\bar{k}_{2}} \dot{\eta}_{\bar{k}_{3}}\left(\frac{1}{2}-\hat{k}_{2} \bullet \hat{k}-\frac{1}{2} \hat{k}_{2} \bullet \hat{k}_{3}\right)\right] .
$$

with $\bar{k}_{3}=\bar{k}-\bar{k}_{2} \cdot k, k_{2}$, and $k_{3}$ are wavenumbers, or magnitudes of the vectors. The unit vectors are defined as:

$$
\hat{k}=\bar{k} / k \quad \hat{k}_{2}=\bar{k}_{2} / k_{2} \quad \hat{k}_{3}=\bar{k}_{3} / k_{3}
$$

The growth rate is not the classical because of the inclusion of surface tension, and is expressed as

$$
\gamma(k)=\sqrt{g k A-\frac{T k^{3}}{\left(\rho_{H}-\rho_{L}\right)}} .
$$

If the second order terms are neglected, the solution to Eq. (28) for an initially stationary fluid is the standard linear result, as derived in the previous section, and is given by

$$
\eta_{\bar{k}}^{\text {lin }}(t)=\eta_{\bar{k}}(0) \cosh [\gamma(k) t]
$$

Even if an exact solution to Eq. (28) including second order terms could be derived, its validity would be questionable, as terms of third order and higher were neglected in its derivation. These terms are certainly as important as the second order terms when the system is fully nonlinear. As a result, Eq. (28) is only valid during the weakly nonlinear phase of perturbation growth. Haan solved the equation approximately by substituting Eq. (29) into the terms on the right hand side of Eq. (28), which simplifies the expression. The result is a bit lengthy, but for most practical ICF applications it may be approximated as 


$$
\eta_{\bar{k}}(t)=\eta_{\bar{k}}^{l i n}(t)+A k \sum_{\bar{k}_{2}} \eta_{\bar{k}_{2}}^{l i n}(t) \eta_{\bar{k}_{3}}^{l i n}(t) 2 G\left(\bar{k}, \bar{k}_{2}\right)
$$

where $G\left(\bar{k}, \bar{k}_{2}\right)$ is given by

$$
G\left(\bar{k}, \bar{k}_{2}\right)=\frac{\frac{1}{2} \gamma^{2}\left(k_{2}\right)\left[1-\hat{k}_{2} \bullet \hat{k}\right]+\frac{1}{2} \gamma\left(k_{2}\right) \gamma\left(k_{3}\right)\left[\frac{1}{2}-\frac{1}{2} \hat{k}_{2} \bullet \hat{k}_{3}-\hat{k}_{2} \bullet \hat{k}\right]}{\left\{\left[\gamma\left(k_{2}\right)+\gamma\left(k_{3}\right)\right]^{2}-\gamma^{2}(k)\right\}} .
$$

Notice how the time and spatial dependence are neatly separated in Eq. (30). The origin of second harmonics is clearly seen in the second order coupling terms of the expression. A condition of applicability of Eq. (30) is that the modes contributing to the summation be adequately modeled by Eq. (29) because Eq. (29) was used to approximate these modes to obtain a solution. The fundamental modes must remain unchanged to second order for the theory to remain valid.

Mode coupling has a significant effect on perturbation growth from the Rayleigh-Taylor instability. Figure 2-3 shows this by applying Haan's model to three different cases of the Rayleigh-Taylor instability: single mode classical, multiple mode classical, and multiple mode stabilized by ablation and a density scale length. The solid lines in the Figure 2-3 represent the growth of an initial perturbation due to linear theory only, whereas the dashed lines show mode coupling results. The significance of the line delineating $1 / k^{2}$ is the boundary between linearity and nonlinearity. As the modes of the spectrum reach this line, they grow more slowly, so there is a gradual progression of the fastest growing mode from high to low order. The fastest growing mode will be the smallest wavelength not 
effectively stabilized or saturated. Thus small structures initially dominate the flow, evolving to larger and larger structures as the dominant wavelength increases.

Figure 2-3 shows mode coupling has a stronger influence on the classical than the ablative Rayleigh-Taylor instability because the fastest growing mode is much smaller in the classical than in the ablative instability. The smaller modes have a shorter e-folding time and form harmonics of both higher and lower frequencies quicker.

For evolution past the weakly nonlinear stage, Haan proposed a second model in which modes do not saturate based on their individual amplitude, but rather on the combined amplitude of modes within some distance $\varepsilon$ from them in wavenumber space. After some analysis, the saturation amplitude was found to be

$$
\eta_{S}(k)=v /\left(L k^{2}\right) .
$$

where $v$ is a parameter taking into account $\varepsilon$ and the amplitude at which the individual modes would saturate. Before a mode saturates, it grows linearly. After saturation, it will continue to grow at a constant rate equal to the linear rate at the time of saturation. This rate is

$$
\eta_{S a t}(k, t)=\eta_{S}(k)\left[1+\ln \frac{\eta_{\text {lin }}(k, t)}{\eta_{S}(k)}\right] .
$$


Figure 2-3: Mode Coupling

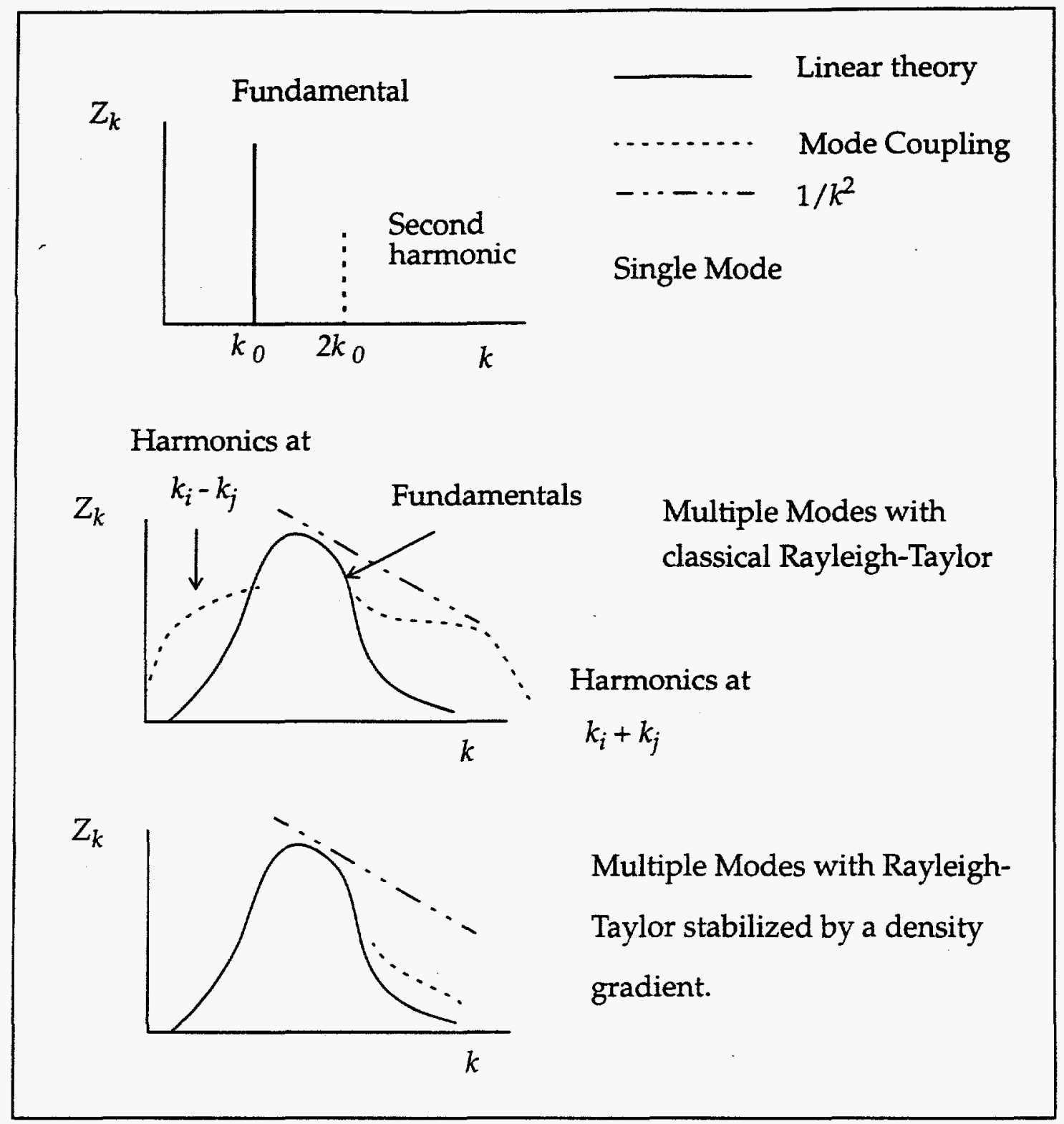

This theory is satisfactory for the ablative Rayleigh-Taylor instability, for which saturation is the most significant nonlinear aspect of the physics. It is thus useful for modeling most ICF plasmas. It is not applicable for classical Rayleigh-Taylor instability problems, as it does not include mode coupling which is the most important nonlinear effect in the classical Rayleigh-Taylor instability. 
Ofer proposed a model combining Haan's mode coupling and saturation theories, which is applicable to both the classical and ablative Rayleigh-Taylor instabilities [Ofer].

After many modes have coupled and saturated, the interface reaches a disordered state where the two fluids mix. Bubbles and spikes have formed and Fourier modes are no longer a valid concept. Youngs computationally investigated the interpenetration of the fluids during this time. He found that the dominant wavelength continued to increase in size, as in the mode-coupling regime. The mechanism may be best understood through bubble competition rather than mode coupling. If a bubble of the lighter fluid is slightly larger than its neighbors, it grows more rapidly and eventually crowds out the surrounding bubbles. When the dominant wavelength reaches about ten times the wavelength of the initially fastest growing mode, the initial system parameters have been forgotten. The evolution could thus be predicted in all cases by simple expressions and no knowledge of original conditions.

A good measure of the evolution of the instability is the width of the mixed region, $\delta$. For a given density ratio, the mixing process is described by a similarity solution with scale length proportional to $g t^{2}$ and may be expressed as

$$
\delta=F\left(\frac{\rho_{1}}{\rho_{2}}\right) g t^{2}
$$

If large amplitude, long wavelength perturbations are present, then the growth of the mixed region will exceed Eq. (31). To define $\delta$, a volume fraction of the dense 
fluid at the point $(x, y, z)$ is used and designated $f_{1}(x, y, z)$. Let the initial interface between the fluids be in the $x-y$ plane, with the $z$ axis normal to the interface. The average of $f_{1}$ at a height $z$ is then given by

$$
\hat{f}_{1}(z)=\frac{\iint f_{1}(x, y, z)(d x) d y}{\iint(d x) d y}
$$

Integration over $d x d y$ refers to integration over the $x-y$ plane. Youngs continued to define the following:

$h_{1}=$ the difference in height between the position the undisturbed interface would have reached and the point where $\hat{f}_{1}=0.99$, or the penetration of the light fluid.

$h_{2}=$ the difference in height between the position the undisturbed interface would have reached and the point where $\hat{f}_{1}=0.01$.

Then one has $\delta=h_{1}+h_{2}$. Computational study and application of Eq. (31) showed

$$
h_{1}=\alpha \frac{\left(\rho_{1}-\rho_{2}\right)}{\left(\rho_{1}+\rho_{2}\right)} g t^{2}
$$

where $\alpha \cong 0.04-0.05$. Eq. (32) was confirmed experimentally, with values of $\alpha$ slightly higher than those derived computationally. Youngs found that the ratio $h_{2} / h_{1}$ increased as the density ratio increased. For the particular multimode calculations he performed he found 


$$
\begin{array}{ll}
\frac{\rho_{1}}{\rho_{2}}=3 & \frac{h_{2}}{h_{1}}=1.5 \\
\frac{\rho_{1}}{\rho_{2}}=20 & \frac{h_{2}}{h_{1}}=2.5
\end{array}
$$

\subsubsection{The Rayleigh-Taylor Instability in Stratified Fluids}

Due to interface coupling, the growth of perturbations, whether from the Rayleigh-Taylor or Richtmyer-Meshkov instability, is different when the fluid is of a thickness on the order of a wavelength. The previous discussions relating to linear and multiple mode growth assumed an interface between two semi-infinite layers, where finite thickness effects were not a concern. Finite thickness effects are important in the feed-out packages, as the wavelength is $50 \mu \mathrm{m}$ and the packages are from 35-86 $\mu \mathrm{m}$ thick. In addition, some of the packages contained a layer of beryllium on the cold side. As the initial densities of beryllium and aluminum are 1.8 and $2.7 \mathrm{~g} / \mathrm{cm}^{3}$ respectively, this resulted in three fluid interfaces where perturbations grew and interacted: the ablated material/aluminum, the aluminum/beryllium, and the beryllium/air interfaces. Because the fluids were compressible, these interfaces were not clearly distinct, but they formed density gradients as one fluid flowed into another. The incompressible theories presented here by Mikaelian and Ott provide insight into the compressible case.

One can clearly see the physics of interface coupling in Mikaelian's incompressible, linear model for stratified fluids [Mikaelian 1982, 1983, and 1995]. For example, take the situation where the densities on either side of a central 
material are the same, giving the density pattern $\rho_{A} / \rho_{B} / \rho_{A}$. In this case, Mikaelian found that

$$
\begin{aligned}
\eta_{1}(t)= & \frac{1}{2} \tan [\vartheta]\left\{\left(\cot \left[\frac{\vartheta}{2}\right] \eta_{1}(0)+\eta_{2}(0)\right) \cos [\gamma t]\right. \\
& \left.+\left(\eta_{2}(0)-\tan \left[\frac{\vartheta}{2}\right] \eta_{1}(0)\right) \cos [\gamma t]\right\} \\
\eta_{2}(t)= & \frac{1}{2} \tan [\vartheta]\left\{\left(\cot \left[\frac{\vartheta}{2}\right] \eta_{2}(0)+\eta_{1}(0)\right) \cos [\gamma t]\right. \\
& \left.+\left(\eta_{1}(0)-\tan \left[\frac{\vartheta}{2}\right] \eta_{1}(0)\right) \cosh [\gamma t]\right\},
\end{aligned}
$$

where $\eta$ is the perturbation on each interface, with the acceleration vector, $g$, pointing from the second to the first interface; $t$ is time, $\gamma$ is the growth rate for the system and $\vartheta$ is a coupling angle, which are both defined by the following set of equations:

$$
\begin{gathered}
\sin [\vartheta]=\frac{2 W\left(z_{1}\right) / W\left(z_{2}\right)}{1+\left(W\left(z_{1}\right) / W\left(z_{2}\right)\right)^{2}} \quad \frac{\gamma^{2}}{g}=\frac{k(R-1)}{\sqrt{1+R^{2}+2 R \operatorname{coth}[k \tau]}} \\
\frac{W\left(z_{1}\right)}{W\left(z_{2}\right)}=1+S \tanh \left[\frac{k \tau}{2}\right]+\frac{S}{R}\left(1+\sqrt{1+R^{2}+2 R \operatorname{coth}[k \tau]}\right) \\
S=\sinh [k \tau] \quad R=\frac{\rho_{B} .}{\rho_{A}}
\end{gathered}
$$

$W$ may be thought of as the $z$ spatial component of the $V_{\pi z}$ term in section 2.2.2. The $z$ coordinate is perpendicular to the plane of the interface; $\tau$ is the thickness of the intermediate fluid layer. Interface coupling may be seen in Eq. (33). $\eta_{1}(t)$ is a function of both $\eta_{1}(0)$ and $\eta_{2}(0)$, as is $\eta_{2}(t)$. Even if $\eta_{1}$ was initially zero, it would 
still grow due to the coupling. An interesting thing happens if one sets $\eta_{1}(0) / \eta_{2}(0)=\tan [\theta / 2]$. The growing modes on both interfaces cancel, and the interfaces become stable. Likewise, if $\eta_{1}(0) / \eta_{2}(0)=\cot [\theta / 2]$, the oscillatory modes cancel. This is a type of freeze-out, which is also seen in the RichtmyerMeshkov interface coupling.

For the system of multiple interfaces, shown in Figure 2-4, the expression for the growth of an individual perturbation is somewhat more complex. A brief review of the derivation of this expression will now be presented.

Mikaelian's model assumes uniform density in each layer and neglects viscosity, surface tension, and heat transfer. The derivation starts with Eq. (17). For this derivation, one may replace $V_{\pi z}$ with just the spatial component in $z$, which will be designated here as $W$. The other two spatial components and time dependence may be canceled out of the equation. To obtain the jump condition,

Figure 2-4: Stratified Fluid Layers.

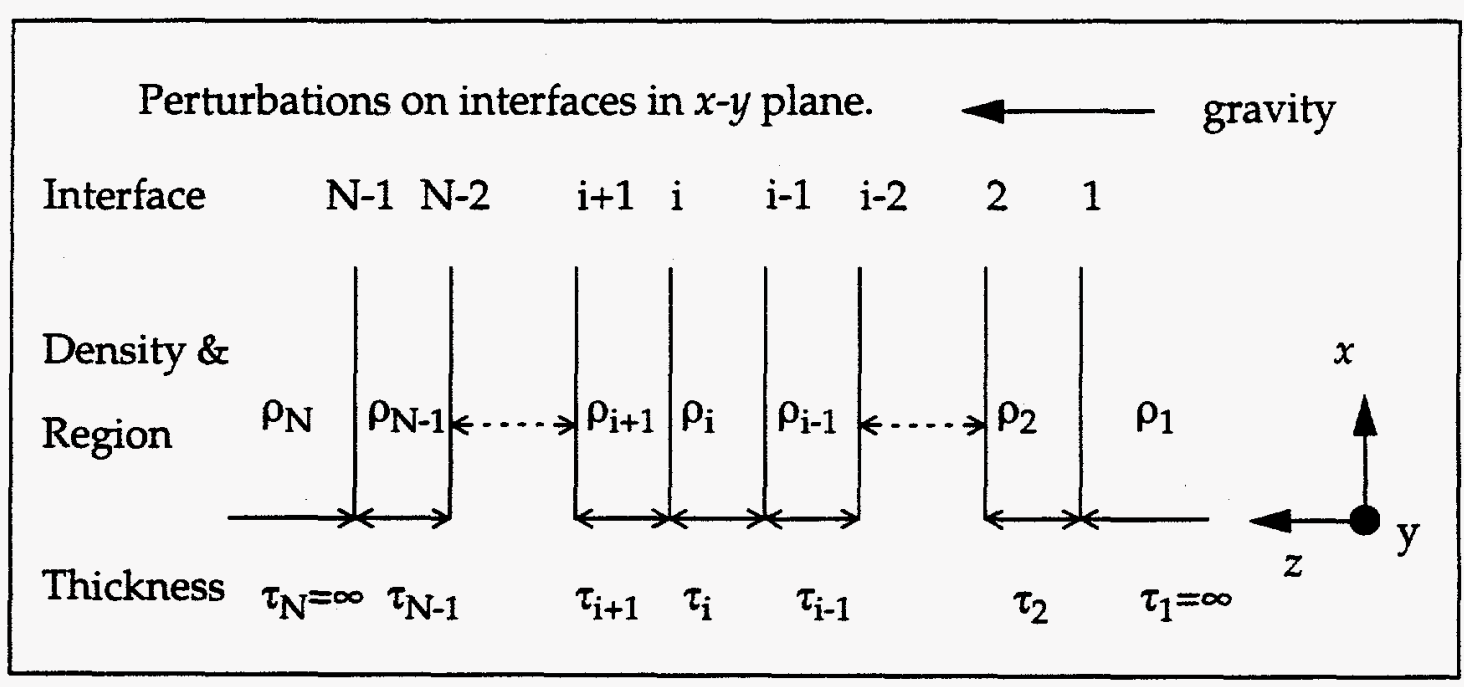


one again integrates Eq. (17) over each interface, but the result for the stratified fluids case is slightly altered from Eq. (18). It is given by

$$
\Delta\left(\rho \frac{\partial W}{\partial z}\right)+\frac{g k^{2}}{\gamma^{2}} W \Delta(\rho)=0,
$$

whére the $\Delta$ operator refers to the difference in the fluid quantities above and below the interface. Eqs. (35) is a system of equations for $N-1$ interfaces and may be written out as:

$$
\begin{array}{rlrl}
B_{1}\left(W\left(z_{1}\right)\right)+C_{1} W\left(z_{2}\right) & =\left(\frac{1}{\chi}\right) W\left(z_{1}\right) & & i=1 \\
A_{i} W\left(z_{i-1}\right)+B_{i} W\left(z_{i}\right)+C_{i} W\left(z_{i+1}\right) & =\left(\frac{1}{\chi}\right) W\left(z_{i}\right) & \\
A_{N-1} W\left(z_{N-2}\right)+B_{N-1} W\left(z_{N-1}\right) & =\left(\frac{1}{\chi}\right) W\left(z_{N-1}\right) & i=N-1 .
\end{array}
$$

The subscripts on $z$ refer to the value of $z$ at the $i$ th interface. The quantities in Eq (36) are defined as:

$$
\begin{aligned}
& A_{i}=\frac{-\rho_{i}}{\sinh \left[k \tau_{i}\right]\left(\rho_{i+1}-\rho_{i}\right)} \quad C_{i}=\frac{-\rho_{i+1}}{\sinh \left[k \tau_{i+1}\right]\left(\rho_{i+1}-\rho_{i}\right)} \quad \chi=\frac{\gamma^{2}}{g k}
\end{aligned}
$$

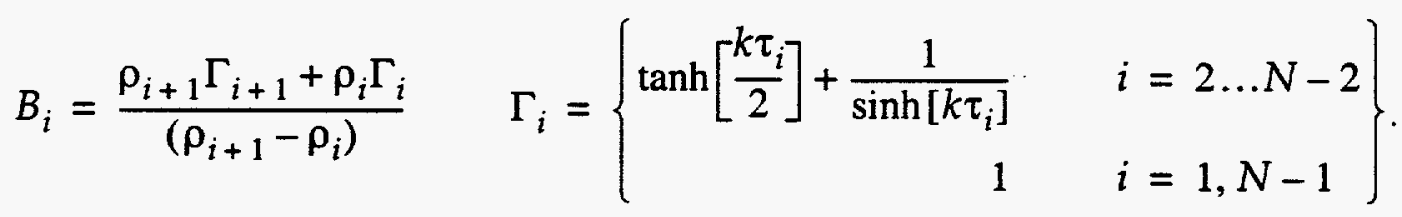

This is an eigenvalue problem with eigenvalues $1 / \chi$ and eigenvectors, $W$, and may be written in matrix form 


$$
M W=\left(\frac{1}{\chi}\right) W
$$

The matrix $M$ is a $(N-1) \times(N-1)$ band matrix with elements $A_{i}, B_{i}, C_{i}$, from Eq. (36); $W$ is an $N-1$ dimensional eigencolumn with elements $W\left(z_{1}\right), W\left(z_{2}\right), \ldots W\left(z_{N-1}\right)$ which are the values of $W$ at the interfaces. There are N-1 eigenvalues, each with its corresponding eigenvector. Unlike the single interface case, the eigenvectors do not correspond to the growth of a particular interface. The eigenvectors with their respective eigencolumns are referred to as normal modes. To obtain the growth at an individual interface, the contribution of all the normal modes to that interface must be summed. The relationship between $W$ and $\eta$ is simply, $W(z)=\partial \eta / \partial t$. Using this and summing over all modes one finds the amplitude at interface $i$ to be

$$
\eta_{i}(t)=\sum_{l=1}^{N-1} \sum_{j=1}^{N-1} \frac{W_{l}\left(z_{i}\right)}{W_{j}\left(z_{l}\right)}\left(\eta_{j}(0) \cosh \left[\gamma_{l} t\right]+\frac{\dot{\eta}_{j}(0)}{\gamma_{l}} \sinh \left[\gamma_{l} \tau\right]\right) .
$$

The $l$ subscript on $W$ and $\gamma$ relates each eigenvector to its respective eigenvalue. The $\gamma$ used here is the one given in Eq. (37).

Mikaelian's theory is only valid in the linear regime, but Ott derived a theory for stratified fluids that is valid into the early stages of the nonlinear regime [Ott]. Unfortunately, the theory is limited in applicability to large Atwood numbers and large $\lambda / \tau$, but may be used to provide insight into the evolution of the feed-out foils after the foils have become thin from ablation. The theory predicts the growth of perturbations on an infinitesimally thin ribbon of fluid sand- 
wiched between massless fluids on either side. The derivation is elegantly simple. Using Newton's second law, Ott wrote an expression for the force on a differential length of the thin film, which included pressure and gravity. From the acceleration, he derived equations for the position as a function of time, which were a set of linear coupled partial differential equations. Solving these, he found the position of a piece of the thin film, originally located at $(x=\xi, y=0)$ was given by

$$
\begin{aligned}
& \bar{r}(x, y, t)=x\left(\xi_{0}, t\right) \hat{x}+y\left(\xi_{0}, t\right) \hat{y} \\
& x\left(\xi_{0}, t\right)=\xi_{0}-\sum_{K, \sigma} \xi_{x}^{\sigma}(K, t) \cos \left(K \xi_{0}+\vartheta_{K}^{\sigma}\right) \\
& y\left(\xi_{0}, t\right)=\sum_{K, \sigma} \xi_{y}^{\sigma}(K, t) \sin \left(K \xi_{0}+\vartheta_{K}^{\sigma}\right),
\end{aligned}
$$

where the functions $\xi_{x}^{\sigma}(K, t)$ and $\xi_{y}^{\sigma}(K, t)$ are solutions to

$$
\ddot{\xi}+K g \xi=0 .
$$

The superscript $\sigma$, labels the four linearly independent solutions of Eq. (40). If one assumes $\xi \sim e^{-i \omega t}$, then there are two oscillatory roots with $\omega= \pm \sqrt{K_{g}}$, one growing root, $\omega=i \sqrt{K g}$, and one decaying root, $\omega=-i \sqrt{K g}$. As the $K$ components do not correspond to a perturbation that is sinusoidal in space, the theory is nonlinear. Because of this, $K$ and $\omega$ are similar to $k$ and $\gamma$ of the linear theory, but not identical.

Ott observed the evolution of a special case of Eqs. (39), with 


$$
\begin{aligned}
& x=\xi_{0}-H \exp (t \sqrt{K g}) \cos \xi_{0} \\
& y=H \exp (t \sqrt{K g}) \sin K \xi_{0},
\end{aligned}
$$

where the initial amplitude is given by $H$. This curve is composed of a number of sinusoidal modes, but as $H$ becomes small, they all disappear except the fundamental $\sin K \xi_{0}$, and the solution approaches linear theory. Eq. (41) evolves into bubbles and spikes, with fluid flowing into the spikes from the bubbles on either side. When the ribbon of fluid folded back on itself forming a loop, the solution became unphysical. Mikaelian showed his linear theory to be equivalent to Ott's for cases of small perturbations [Mikaelian 1996].

Compressibility effects in stratified fluids are important for a more accurate understanding of the real systems encountered in experiment. Yang and Zhang theoretically studied general properties of stratified incompressible and compressible fluids using the linearized Euler equations [Yang 1993]. Their work provides some insights into compressibility effects in such systems. They proved that all eigenvalues, or perturbation growth rates, for a stratified fluid system are real and nondegenerate. They showed that a system of compressible fluids is always more unstable than an incompressible system with the same equilibrium density distribution. Two necessary and sufficient conditions for stability of a compressible system are for there to be no density inversions on any interface, and for 


$$
\frac{g}{\rho} \frac{\partial \rho}{\partial z}-\frac{g^{2}}{c_{S}} \geq 0
$$

where $c_{S}$ is the sound speed. Stability is assured in a compressible system by a lack of density inversions on the interfaces. The maximum growth rate for any multilayer system with no surface tension, a free lower boundary and either a fixed or free upper boundary, is $\gamma=\sqrt{|g| / k}$.

Considering the multimode work of Haan in the light of the stratified fluid work presented in this section, one may realize that any harmonic modes generated by nonlinearity should couple across the interfaces. Interface coupling of the harmonics could result in the system approaching nonlinearity faster, as there would be additional second order terms in Haan's mode coupling expression. Such a coupling was not experimentally observed in the feed-out experiments, probably because the ablative stabilization discouraged harmonic growth on the hot surface.

\subsubsection{The Richtmyer-Meshkov Instability}

If a shock passes through a perturbed interface, the perturbation will usually grow. This is the Richtmyer-Meshkov instability. The effect of the shock is to briefly accelerate the perturbation, giving it a velocity with which it coasts afterward. This instability occurs in the feed-out packages when a shock created by hohlraum radiation interacts with a perturbation on the cold surface of the foil.

The growth of a perturbation under the influence of the Richtmyer- 
Meshkov instability may be estimated by applying the linear Rayleigh-Taylor instability model to the perturbation during the acceleration period of the shock. The perturbation will then grow according to

$$
\eta(t)=\eta_{0} e^{\gamma t}
$$

Take the second derivative of this expression with respect to time, then make the assumption that the time period during which the acceleration is nonzero is very small, such that $t \cong 0$ during this period. Then the exponential goes to one and we have the expression

$$
\frac{d^{2} \eta}{d t^{2}}=\eta_{0} g k A
$$

Integrating twice gives the perturbation amplitude as a function of time

$$
\frac{\eta(t)}{\eta_{0}}=1+k A v_{s} t
$$

where $v_{s}$ is the difference in fluid velocity across the shock. This simple analysis of the Richtmyer-Meshkov instability is referred to as the impulsive model and was first presented by Richtmyer [Richtmyer]. There are some difficulties with the impulsive model. It is only valid in the linear regime. The impulsive model also predicts immediate growth of the perturbation after shock passage. Of course this does not happen because the velocity imparted to the trough and crest of the perturbation are equal. Also, the shock compresses the perturbation and both fluids as it passes by. The amplitudes before and after shock passage are thus different, leading to some ambiguity in the expression. 
Yang, Zhang, and Sharp compared the impulsive model to computational solutions of the linearized hydrodynamic equations, which are more accurate [Yang 1994]. They found the best agreement by using post-shock amplitudes for the reflected shock case and the average of the post- and pre-shock amplitudes for the reflected rarefaction. For the weak shock limit, the impulsive model and linear theory provide the same solution, but they diverge as shock strength increases. The agreement is also improved as the adiabatic exponents of the two fluids increase, while remaining approximately equal to one another. Good agreement was found to be particularly true for the reflected rarefaction case. The largest discrepancies occur when the adiabatic exponents are substantially different and the shock is very strong.

The impulsive model was presented here despite its flaws because it provides a clear insight into Richtmyer-Meshkov instability physics. For a more rigorous treatment of the instability, one may apply linear perturbation theory directly to the Richtmyer-Meshkov problem. First, a solution to the Riemann problem must be obtained, which is simply the problem of a shock hitting a flat interface. The hydrodynamic equations are then linearized around the solution to the Riemann problem. This two step process is necessary because the differential form of the hydrodynamic equations is not valid at the jump interface created by a shock, but only in the fluid on either side of a shock.

There is no closed form analytic solution to the Riemann problem, but there is a way to solve it geometrically by constructing a wave diagram in the 
pressure-velocity plane, $p-v$ plane, see Figure 2-5 [Yang 1994]. One can show for a given state of the fluid ahead of a shock, the velocity behind the shock is given by

$$
v=v_{a}-\left(p-p_{a}\right)\left(\frac{\rho_{a}}{2}\right)^{-\frac{1}{2}}\left[\left(\gamma_{G}+1\right) p+\left(\gamma_{G}-1\right) p_{a}\right]^{-\frac{1}{2}} .
$$

Figure 2-5: Riemann Problem

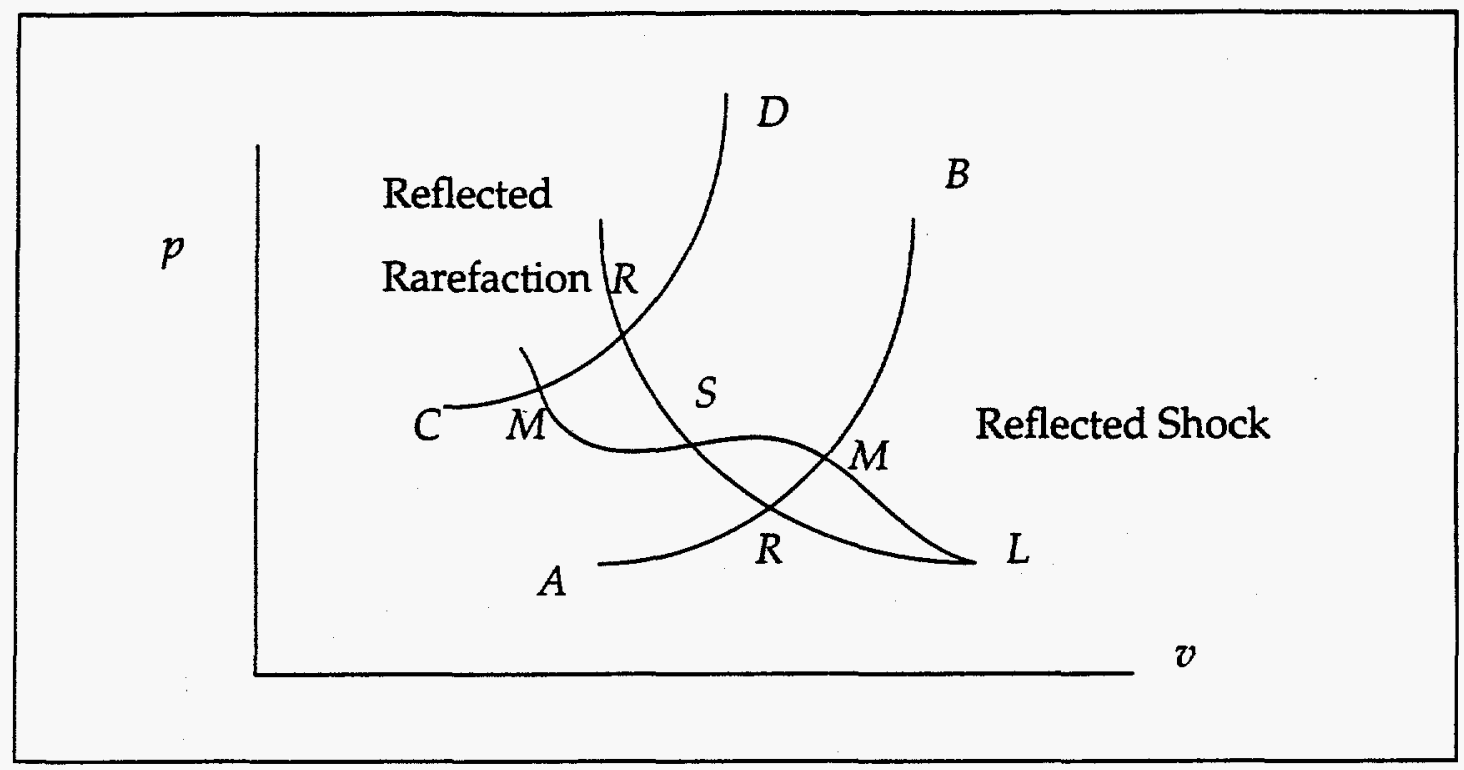

The subscripts $a$ refer to the state ahead of the shock, whereas quantities without subscripts represent those behind the shock; $\gamma_{G}$ is the adiabatic exponent of the gas. Eq. (42) is a curve in the $p-v$ plane. Now consider what happens when a shock hits a discontinuity. There will be a transmitted shock, and either a reflected rarefaction or shock. Both the reflected and transmitted waves will have curves given by Eq. (42) on the $p-v$ diagram. Curve $M L$ is the curve for the transmitted shock, $R L$ is for the incident shock, and $A B$ is the reflected wave. The states of the fluid behind the transmitted shock and reflected wave must be the same. Thus, 
the solution to the Riemann problem for the state of the material at the interface is the intersection of the two curves on the diagram, at point $M$. If point $M$ is above $R$, as in the case shown for the curve $A B$, then the reflected wave is a shock. If $M$ was below $R$ on $A B$, a rarefaction would be the result.

Sometimes curves $M L$ and $R L$ cross at a point $S$, as shown in the figure. In such a case, as shock strength increases, $A B$ moves upward to the position $C D$. On $C D, M$ is below $R$, meaning the reflected wave is a rarefaction. Thus, whether a shock or rarefaction is reflected depends on the strength of the incident shock.

The nature of the reflected wave may be determined as follows. In general, if the incident shock moves from low to high impedance, $\gamma_{G 2} \rho_{2} \rightarrow \gamma_{G 1} \rho_{1}$, then $M$ is above $R$ and a shock is reflected. From high to low, a rarefaction results and is referred to as "normal reflection." If the material properties of the two gases satisfy inequality (43)

$$
\begin{array}{ll}
\gamma_{G 1}<\gamma_{G 2} \rightarrow & \frac{\gamma_{G 1}}{\gamma_{G 2}} \leq \frac{\rho_{2}}{\rho_{1}} \leq \frac{\gamma_{G 1}+1}{\gamma_{G 2}+1} \\
\gamma_{G 1}<\gamma_{G 2} \rightarrow & \frac{\gamma_{G 1}+1}{\gamma_{G 2}+1} \leq \frac{\rho_{2}}{\rho_{1}} \leq \frac{\gamma_{G 1}}{\gamma_{G 2}},
\end{array}
$$

then the situation is more complex, and curves $\mathrm{RL}$ and $\mathrm{ML}$ cross at the critical shock strength of

$$
S=\frac{2\left(\gamma_{G 1} \rho_{1}-\gamma_{G 1} \rho_{1}\right)}{\left(\gamma_{G 1}-1\right) \rho_{1}-\left(\gamma_{G 2}-1\right) \rho_{2}}=\frac{p-p_{a}}{p}
$$

The subscripts 2 and 1 refer to the material the shock is moving from and into respectively. If $A B$ is below point $S$, normal reflection occurs, but if it is 
above, then normal reflection is reversed. If the shock strength is precisely $S$, then the impedances of the gases exactly match, and there is no reflected wave in linear analysis.

If there is an initial perturbation at the interface, then the shock or rarefaction reflected from the interface will also carry a perturbation. The reflected shock or rarefaction is the leading edge of the flow field of the Richtmyer-Meshkov instability. Fluid in the flow field will be imprinted with the perturbation. A perturbation on a shock will decay with time and distance away from the interface, but a perturbation on a rarefaction will neither grow nor decay. A stable perturbation on a rarefaction implies that the imprint on the ablation surface could be larger when the reflected wave is a rarefaction than a shock. The reflected wave in all the feed-out experiments was a rarefaction.

The perturbations at the interface may undergo one of two types of phase inversion: direct and indirect. Direct phase inversion is caused directly by the shock-interface interaction, and phase inversion occurs at or before the shock has passed the interface. In indirect phase inversion, the perturbations are not phase-inverted immediately after the shock has passed, but are moving in the direction of inversion. Later in the evolution of the interface, they invert. Direct inversion cannot occur when the reflected wave is a shock and only occurs when the reflected rarefaction is sufficiently strong. Indirect inversion rarely if ever occurs when the reflected wave is a shock. Thus, for a direct phase inversion to occur, the reflected wave must be a rarefaction. 
Freeze-out is a condition where the perturbations neither grow nor decay after shock passage. This type of freeze-out should not be confused with freeze-out from interface coupling. No perturbation growth occurs because the shock reflected from the interface exactly cancels the effects of the shock transmitted through the interface. This situation can occur for both reflected rarefactions and shocks, but it cannot occur if the two adiabatic exponents are equal. It is believed impossible for freeze-out and total transmission to occur at the same time.

Just as the impulsive model is not as rigorous as the linear model, the linear equations are not as accurate as the nonlinear hydrodynamic equations. Each model predicts a different growth rate with time. To study the differences between these models, Holmes, Grove and Sharp computationally investigated the Richtmyer-Meshkov instability between air and $\mathrm{SF}_{6}$, and between air and $\mathrm{He}$ [Holmes]. They compared the results of the linear model to nonlinear hydrodynamic equations. The shock strength was Mach 1.2 in air, and the perturbations $0.24 \mathrm{~cm}$ in amplitude and $3.75 \mathrm{~cm}$ in wavelength. A sketch of their general results is shown in Figure 2-6. Twice there were deviations of the linear from the nonlinear results at 70 and $200 \mu \mathrm{s}$. Both of these deviations result in a decrease in the growth rate from the linear prediction and are generated by the creation of secondary shocks from nonlinear interactions. The secondary shock interaction at $200 \mu \mathrm{s}$ is so strong, it results in a permanent deceleration of the bubble. In addition, the linear solution contains acoustic waves, whereas in the nonlinear case 
Figure 2-6: Typical Richtmyer-Meshkov Growth Rate

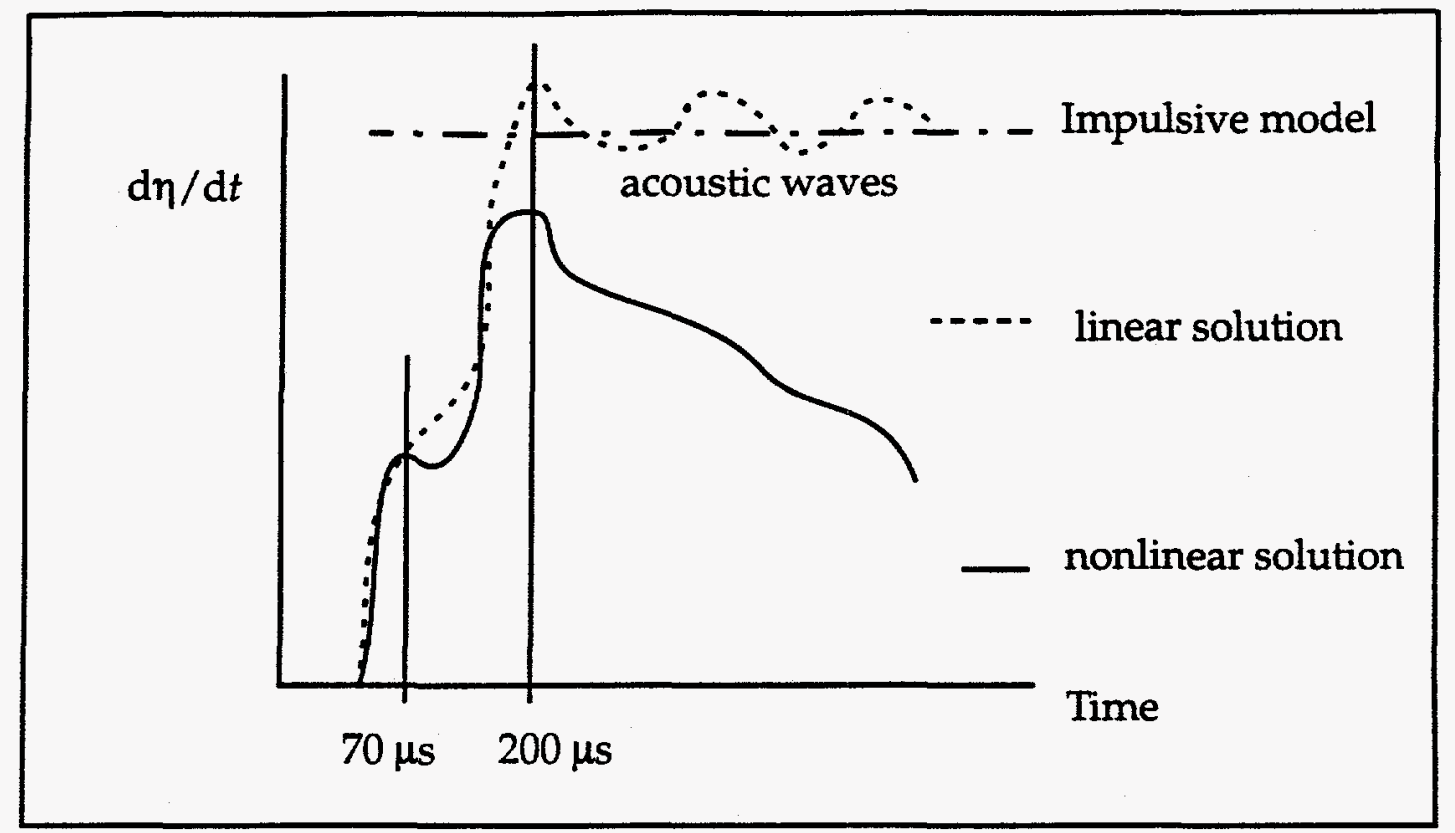

these waves may steepen into secondary shocks. There are two important features of the linear solution causing these differences. Characteristics are not allowed to focus, creating secondary shocks, and linearization constrains the geometry of the wave fronts to be sinusoidal.

As with the Rayleigh-Taylor instability, mode coupling occurs in the Richtmyer-Meshkov instability. Mode coupling is slower in a Richtmyer-Meshkov instability because the fluid is under acceleration for a much shorter time than in the Rayleigh-Taylor instability. Given sufficient time, the harmonics combine to form bubbles and spikes. Haan extended his mode coupling model for the Rayleigh-Taylor instability to the Richtmyer-Meshkov instability [Haan August 1991]. The impulsive model was used, with the assumption that $g=0$ and the perturbation is coasting at some constant velocity, which leaves the derivation of Eq. 
(28) unchanged. The solution is

$$
\eta_{\bar{k}}(t)=\eta_{\bar{k}}^{l i n}(t)+\frac{1}{2} A k \sum_{\bar{k}_{2}} \eta_{\bar{k}_{2}}^{l i n}(t) \eta_{\bar{k}_{3}}^{l i n}(t)\left[\frac{1}{2}-\hat{k}_{2} \bullet \hat{k}-\frac{1}{2} \hat{k}_{2} \bullet \hat{k}_{3}\right]
$$

assuming the dominant modes have been growing long enough that

$$
\eta_{\bar{k}}^{l i n}(t) » \eta_{\bar{k}}^{\text {lin }}(0)
$$

During late times, the bubble and spike velocities may be described by the power law, $v=c t^{-\alpha}$ where $c$ and $\alpha$ are some constants [Holmes].There is a sharp contrast between these exponentially decaying velocities of the RichtmyerMeshkov and Rayleigh-Taylor instabilities. In the Rayleigh-Taylor instability, the bubble approaches a constant velocity and the spike undergoes free fall for high Atwood numbers.

\subsubsection{The Richtmyer-Meshkov Instability in Stratified Fluids}

If there are multiple fluid interfaces within the distance of several perturbation wavelengths of each other, a Richtmyer-Meshkov instability on one will couple with all the other interfaces. The situation with multiple interfaces is shown in Figure 2-4, with the gravity vector pointing in the direction of acceleration from the shock. Even if an interface is stable by itself, coupling with the unstable interfaces will produce perturbation growth. This is analogous to the Rayleigh-Taylor interface coupling discussed in section 2.2.3. Richtmyer-Meshkov interface coupling is one of the two mechanisms by which the perturbation on the 
cold surface of a radiation driven foil feeds-out to the ablation surface. It is thus important in understanding the feed-out experiments. The second mechanism, differential acceleration, will be discussed in section 2.2.7.

A theoretical basis is always a good start for the study of any phenomenon of interest. Mikaelian extended his stratified fluid theory to the RichtmyerMeshkov instability using the impulsive model [Mikaelian 1985, 1995]. The result is a simple expression that clearly shows the physics of interface coupling but does not address such complexities as compression or radiation. Taking the second derivative of Eq. (38) and replacing $g$ with $v_{s} \delta\left(t-t_{s}\right)$ where $t_{s}$ is the shock arrival time, then integrating twice gives

$$
\eta_{i}(t)=\eta_{i}(0)+\dot{\eta}_{i}(0) t+v_{s} \sum_{l=1}^{N-1} \sum_{j=1}^{N-1} \Gamma_{l}^{2} \frac{W_{l}\left(z_{i}\right)}{W_{j}\left(z_{l}\right)}\left(\eta_{j}(0)+\dot{\eta}_{j}(0) t_{s}\right)\left(t-t_{s}\right) \Theta\left[t-t_{s}\right]
$$

There are some problems with this expression. It assumes the same velocity is imparted to each interface by the shock, which is unrealistic. As the shock interacts with each interface, either a reflected shock or rarefaction will result, which will interfere with the growth on other interfaces. Also, Eq. (44) assumes the velocity from the shock is imparted at the same time to all the interfaces, which is unrealistic. To apply Eq. (44), the acoustic impedances of the interfaces must match or be very close, and the time necessary for the shock to pass through the various layers must be short compared to the growth rates of the instabilities. Nevertheless, one may use Eq. (44) to learn about interface coupling of the Richtmyer-Meshkov instability. 
By taking a two-interface problem where the densities on each side of the middle layer are the same in the pattern $A / B / A$, Eq. (44) simplifies considerably to

$$
\begin{aligned}
& \eta_{1}(t)=\eta_{1}(0)+\frac{v_{s} \Gamma^{2}}{\cos [\vartheta]}\left(\eta_{1}(0)-\sin [\vartheta] \eta_{2}(0)\right) t \\
& \eta_{2}(t)=\eta_{2}(0)+\frac{v_{s} \Gamma^{2}}{\cos [\theta]}\left(\eta_{2}(0)-\sin [\vartheta] \eta_{1}(0)\right) t
\end{aligned}
$$

where $\theta$ is a coupling angle. $\theta$ is again defined by Eq. (34). Subscripts 1 and 2 refer respectively to the first and second interfaces the shock hits.

Eq. (45) has many interesting characteristics. Interface coupling and feedthrough can easily be observed here. In addition, if $\eta_{1}(0) / \eta_{2}(0)=\sin [\theta]$, then the first interface does not grow. Likewise, if $\eta_{2}(0) / \eta_{1}(0)=\sin [\vartheta]$, the second interface does not grow. The freeze-out described here for the RichtmyerMeshkov instability is similar to freeze-out in Rayleigh-Taylor interface coupling, with the exception that only one of the two interfaces may be frozen at the same time. Freeze-out with the Richtmyer-Meshkov instability has been computationally observed but remains to be experimentally verified [Mikaelian 1996].

As an educational exercise, the growth rates of three cases, the second of which corresponds to the feed-out experiments, are considered using Eq. (45):

Case $A: \eta_{1}(0)=\eta_{2}(0)=\eta_{0}$ sinuous shape,

Case $B: \eta_{1}(0)=\eta_{0} \quad \eta_{2}(0)=0$,

Case $C: \eta_{1}(0)=-\eta_{2}(0)=\eta_{0}$ varicose shape. 
A density ratio of $\rho_{B} / \rho_{A}=3$ is used and a normalized growth rate for the $i^{\text {th }}$ interface is defined as, $N G R_{i}=\dot{\eta}_{i} /\left(\eta_{0} v_{s} k\right)$. The normalized growth rate is the coupled growth rate divided by the growth rate of an uncoupled interface with an Atwood number of one. Because the fluid interfaces in this example do not have an Atwood number of one, the normalized growth rate is not exactly unity when the interfaces are growing classically. Figure 2-7 shows a sketch of approximately how this value varies with $k \tau$. For $k \tau=10$, all of the interfaces have decoupled, and the classical growth rates are observed, but for $k \tau=0.01$, the interfaces have completely coupled, and have the same growth rates. Case $A$ turns into a varicose shape after shock passage, as the interfaces grow in opposite directions. Freeze-out occurs in this case for low $k \tau$. Case $B$ is representative of the situation in the feed-out experiments. As $k \tau$ becomes small in this case, interface 2 begins to grow with interface 1 , even though it has no perturbation, producing a sinuous shape which was experimentally verified with face-on radiography. The growth rates actually increase over the classical value as the two interfaces couple. The growth rates in Case $C$ are also larger than classical for strong interface coupling. Both interfaces always have the same growth rate in this case. Case $C$ also becomes sinuous, but more strongly so than Case $B$. One can see that interface coupling can either increase or decrease the classical growth rate depending on the situation. 
Figure 2-7: Sketch of Normalized Growth Rate

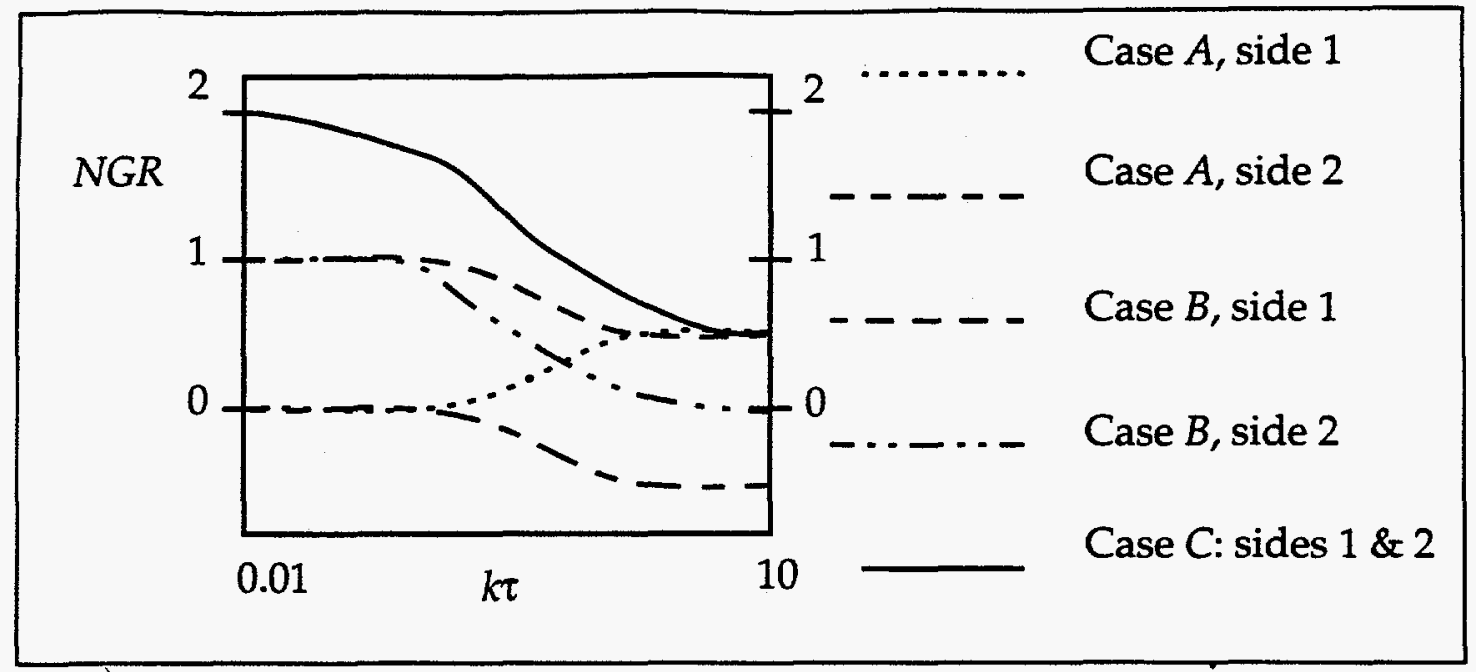

Nonlinearity, compressibility, and density gradient effects have significant influence on interface coupling with the Richtmyer-Meshkov instability but are not included in Mikaelian's theory. All three of these effects are present in the feed-out packages. Systems with these characteristics are too complex to be analyzed with theory, and are always either computationally or experimentally studied. A paper by Baltrusaitis presented calculations and experiments performed in order to study the Richtmyer-Meshkov instability in thin films that were nonlinear, compressible, and had a density gradient [Baltrusaitis]. $\mathrm{SF}_{6}$ gas flowed through a contoured nozzle into a shock tube filled with air. Both sides of the nozzle were corrugated, producing a gas curtain with a varicose cross section and diffusive boundaries. The exact shape of the perturbations could not be controlled precisely from shot to shot, but the initial conditions were recorded before each shot. The dominant wavelength of the initial perturbation was about $6 \mathrm{~mm}$, while the curtain thickness was around $3 \mathrm{~mm}$. The shock was a Mach 1.2. Diffusion of 
the gas formed a density gradient at the fluid interfaces, similar to the density gradient formed in the feed-out packages from preheat.

Three different types of flow pattern were observed and modeled. Upstream mushrooms developed when the initial perturbations were predominantly on the upstream side of the gas curtain, or when the amplitude of the upstream perturbation was much greater than the downstream perturbation. Likewise, downstream mushrooms evolved from a large perturbation on the downstream side of the curtain. A sinuous pattern developed with no mushrooms when both interfaces had roughly equal perturbation amplitudes, or the downstream side was slightly larger. The gas curtain remained intact between the mushrooms, connecting them in a bubble formation. Because $\mathrm{SF}_{6}$ is heavier than air, the shock on the upstream side generated growth but no phase inversion. The shock created growth and phase inversion of the downstream perturbations. Thus, for an initially varicose curtain, the phase of the downstream side would be inverted to match the upstream, forming a sinusoidal pattern.

These results may be understood by considering vortex dynamics. As the pressure gradient from the shock interacts with the density gradient at the interfaces, it generates vorticity. The strength of this vorticity is directly proportional to the amplitude of the perturbations. Thus, the interface with the largest amplitude has the largest vorticity, and dominates the flow. Computational analysis showed that only one pair of vortices were in the gas curtain, the competing vortex pair from the weaker interface having been overcome by the larger. Even 
in the sinuous case, where both amplitudes were equal, there was only one vortex, albeit a much weaker vortex than those of the mushroom curtains.These results may also be understood from an interface coupling standpoint. When the ratio of thickness to wavelength is small, Mikaelian's interface coupling theory predicts that the perturbation grows on the entire ribbon of fluid as a unit, not separately on the interfaces. This was certainly the case with the gas curtain experiments and is the reason for the gas curtain having to "select" one vortex pair to dominate its evolution. A very similar selection between the Rayleigh-Taylor and Richtmyer-Meshkov vortices was observed in the feed-out calculations and is described in chapter 5 .

Mikaelian computationally modeled the experiments in the Baltrusaitis paper and predicted an additional flow pattern that was not observed in the experiments [Mikaelian 1996]. If the curtain was initially sinuous, Mikaelian predicted that both upstream and downstream mushrooms would develop. Perturbations are initially in phase in a sinuous pattern. The shock reversed the phase of the downstream perturbation creating a varicose pattern, which created double mushrooms as it continued to expand.

So far, Richtmyer-Meshkov and Rayleigh-Taylor interface coupling has been discussed as two separate issues, but the two are a coupled system in the feed-out process. The only publications found investigating such a coupled system were by Bel'kov [Bel'kov]. Calculations were performed on laser driven foils with an initial perturbation on the cold side, very similar to the packages 
described in this dissertation. Foil thicknesses of 3 and $5 \mu \mathrm{m}$ were selected, with perturbation wavelengths of 10 and $2 \mu \mathrm{m}$. Calculations were first run with these modes on the hot surface of the package, then on the cold surface to observe the difference in results. Both single and multiple modes were studied to observe mode coupling in feed-out. Ott's model agreed with the computational results very well, both for initial perturbations on the front as well as on the back. The applicability of Ott's theory indicates the foil was sufficiently thin compared to the wavelength that both interfaces were strongly coupled and grew at the same rate. Qualitatively, it was shown that the interaction of the two modes differed somewhat when the perturbations were placed on the hot or cold surfaces. The difference was caused by acoustic-gravity waves being generated in the case of perturbations on the cold surface, but not generated when the perturbations were initially on the ablation surface. Acoustic-gravity waves are part of the RichtmyerMeshkov instability and are a subject discussed in the next section. Unlike the Bel'kov calculations, the feed-out foils move from states where the RichtmyerMeshkov and Rayleigh-Taylor instabilities are not coupled to the state where there is complete coupling and the Rayleigh-Taylor instability dominates.

\subsubsection{Atmospheric Type Modes}

The calculations and experimental data both suggest that all the feedout packages have an internal oscillatory mode which interacts with the instabilities. Because of the acceleration of the foils, there are at least three important inter- 
nal modes allowed by the physics. These are the acoustic, gravity, and Lamb modes, which are all present in the atmosphere. After these three candidates had been identified the task was to determine which mode was present in the experiments. The acoustic mode is usually associated with the Richtmyer-Meshkov instability as sketched in Figure 2-6 so it was initially considered the most likely candidate. Indeed, later calculations indicated that the mode in the feed-out packages was probably acoustic; however, a short description of each is presented here because it is necessary to understand how to differentiate between the three modes. In addition, the physics of the packages allows for all three, and any one of them could be found in future experiments similar to the ones presented here.

The acoustic mode has a different dispersion relation than classical acoustic waves under the influence of acceleration. A general dispersion relation for the acoustic and gravity modes is presented in Gossard and Hooke [Gossard]

$$
\left(\frac{N^{2}}{\omega^{2}}-1\right)\left(1-\frac{\omega^{2}}{k^{2} c_{S}^{2}}\right)=\frac{\Gamma^{2}}{k^{2}} .
$$

The symbol $N^{2}$ is called the Väisälä-Brunt frequency and is given by

$$
N^{2}=-g\left(\frac{1}{\rho} \frac{\partial \rho}{\partial z}+\frac{g}{c_{S}^{2}}\right),
$$

while $\Gamma$ is termed the Eckart coefficient,

$$
\Gamma=\frac{1}{2 \rho} \frac{\partial \rho}{\partial z}+\frac{g}{c_{S}^{2}}
$$

Eq. (46) was originally derived as the dispersion relation for internal oscillatory 
modes propagating in the atmosphere and is for the special case of a nonrotating earth. The $z$ coordinate is altitude. Because the density and sound speed are a function of altitude, the Eckart coefficient and Väisälä-Brunt frequency are also. The expression assumes no vertical component to the propagation.

The atmosphere is much like the feed-out foils and ICF packages in general. It is a compressible fluid, under the influence of a gravitational field, and decreases in density with altitude just as the ablation and cold surfaces of the foil decrease in density as one moves away from the center. There are four roots to Eq. (46) which are forward and backward propagating acoustic and gravity modes.

The gravity mode may be understood in terms of an internal RayleighTaylor instability. In incompressible fluids, the Rayleigh-Taylor instability is a mode that oscillates as a stable wave if $\gamma$ is imaginary, or, if $\gamma$ is real, has growing and decaying parts. In either case, the Rayleigh-Taylor instability exists on the interface of the fluids and is driven by gravity. Any penetration into the fluids of a growing perturbation drops off exponentially from the interface. In a compressible fluid, the mode may exist inside the fluid itself in which case it is an internal gravity wave. To visualize this, imagine a fluid particle in a gas which has a variable density with height due to a gravitational field. If the fluid particle rises, its pressure will drop to equilibrate with the surrounding fluid, but it will still be slightly denser than the fluid at that height. As a result, it will be pulled by gravity back to equilibrium, and due to inertia, pass the equilibrium position, moving lower to a position of buoyancy. The oscillation may be stable, or increase in 
amplitude with time depending on the fluid conditions.

An approximate sketch of the solutions to Eq. (46) is shown in Figure 2-

8. The acoustic branch of Eq. (46) is given by the conditions $\omega^{2} / k^{2}>c_{S}^{2}$ and $\omega>N$. It approaches the classical acoustic dispersion relation for large $k$, For small $k$, it has a cutoff frequency, $N_{A}^{2}=N^{2}+c_{S}^{2} \Gamma^{2}$, below which it does not propagate. The gravity branch is given by the conditions $\omega^{2} / k^{2}<c_{S}^{2}$ and $\omega<N$. It does not propagate above the Väisälä-Brunt frequency, which it asymptotically approaches as $k$ becomes large. One may see that differences in the dispersion relations is the key to distinguishing between the acoustic and gravity modes. The Lamb mode is defined by the intermediate condition for a nonrotating earth, $\omega^{2} / k^{2}=c_{S}^{2}$, which is just the classical acoustic dispersion relation. Because of this, the Lamb mode is not always easily distinguishable from the acoustic mode

Figure 2-8: Atmospheric Type Modes

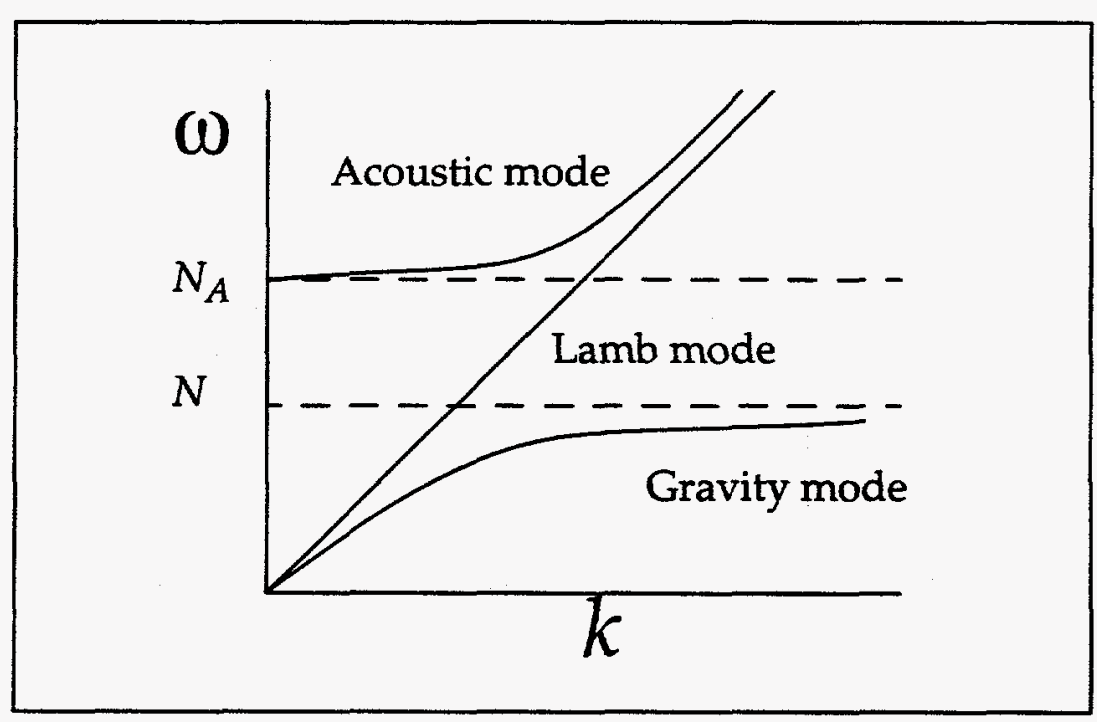


based on the dispersion relation alone.

Depending on the state of the fluid, the gravity and Lamb modes may have a real component, making them unstable. The first to investigate this in relation to inertial fusion plasmas was Scannapieco [Scannapieco]. He performed a linear perturbation analysis on the equations of hydrodynamics, similar to the one in section 2.2.2, with two important differences. He assumed a zeroth order density variation in the direction of gravity of, $\rho_{0}(z)=\rho_{0}(0) e^{z / H}$ where $H$ is a scale length, and he assumed compressibility. The equivalent expression to Eq. (19) was no longer second-order, but rather an eighth-order polynomial in $\gamma$, admitting complex conjugate pairs of gravity, acoustic, and Lamb modes. As the value of $H$ changed, so did the stability of the modes. Table 2-1 shows the stable and unstable modes. The symbol $\gamma_{G}$ is the adiabatic gas constant. The acoustic modes are always oscillatory. The classical Rayleigh-Taylor instability is the growing internal gravity mode for $H>0$, however, the growing gravity mode for $H>-$ $c_{s}^{2} / g$ is not a Rayleigh-Taylor mode. The Lamb mode is the most interesting, as it has a real component to the growth rate for all but the specific case of $H=-c_{s}^{2} /$ $\gamma_{G} g$. The Lamb mode is almost always unstable. 
Table 2-1: Mode Stability

\begin{tabular}{|l|l|l|l|}
\hline \multicolumn{1}{|c|}{$H$} & Acoustic & \multicolumn{1}{c|}{ Gravity } & \multicolumn{1}{c|}{ Lamb } \\
\hline$H>0$ & oscillatory & growing & growing-oscillatory \\
\hline$H>-c_{s}{ }^{2} / \gamma_{G} g$ & oscillatory & oscillatory & growing-oscillatory \\
\hline$H=-c_{s}{ }^{2} / \gamma_{G} g$ & oscillatory & oscillatory & oscillatory \\
\hline$H<-c_{s}{ }^{2} / \gamma_{G} g$ & oscillatory & oscillatory & growing-oscillatory \\
\hline$H>-c_{s}{ }^{2} / g$ & oscillatory & growing & growing-oscillatory \\
\hline
\end{tabular}

Scannapieco's results have important ramifications for the feed-out foils and ICF capsules. They imply that internal fluid instabilities may exist in addition to the Rayleigh-Taylor and Richtmyer-Meshkov surface instabilities. The internal instabilities could be disruptive or benign to capsule performance. Scannapieco's paper indicated that Lamb instabilities would probably saturate rapidly after growth in the linear phase. Thus, to distinguish between acoustic and Lamb modes, one looks at the linear behavior. If there is growth and an acoustic-like dispersion relation, the mode is probably a Lamb mode.

\subsubsection{Differential Acceleration}

Feed-out of a perturbation from the cold to the ablation surface occurs by at least two mechanisms. Richtmyer-Meshkov interface coupling, which was described in section 2.2.6, is one mechanism, and differential acceleration is another. Both of these effects result in a perturbation on the ablation surface $180^{\circ}$ 
out of phase with the original perturbation on the cold surface. Both differential acceleration and interface coupling seed the Rayleigh-Taylor instability and growth on the ablation surface begins.

Differential acceleration may be understood by considering two columns of fluid, one running through the trough of the initial perturbation and the other running through the peak. The column of fluid running through the peak will have a greater mass. Because the force of ablation from the $\mathrm{X}$-ray drive is uniform across the hot surface, the acceleration of the thinner column will be greater than that of the thicker one. As the thinner column begins to move past the thicker, a perturbation will appear on the hot surface.

A simple expression predicting the feed-out from differential acceleration is easily derived [Hoffman 1997], see Figure 2-9. The mean acceleration of the foil is $g=F / m$ where $F$ is the ablative force and $m$ the average foil mass. The mean position of the hot surface, $x(t)$, neglecting ablation, is $x(t)=g t^{2} / 2$. The ratio of the difference in mass of the fluid columns, $\Delta m$, to the average mass may be written, $\Delta m / m=\eta_{0} / \tau$. Combining these three equations, the amplitude of the perturbation on the front surface is $\eta_{D A}(t)=\Delta x(t)=x(t) \eta_{0} / \tau$. Differentiating to obtain the growth rate, one finds, $\dot{\eta}_{D A}(t)=\dot{x}(t) \eta_{0} / \tau=v_{P S} \eta_{0} / \tau$, where $v_{P S}$ is the post shock velocity of the fluid. The acoustic waves generated by the Richtmyer-Meshkov instability will cause the differential acceleration to saturate as the cold surface perturbation phase inverts. Saturation is expected to occur on a 
time scale on the order of the frequency of the acoustic wave, which is approximately $t_{P I}=\lambda / c_{S}$. The maximum growth from differential acceleration is then $\eta_{D A m a x}=\left(v_{P S} / c_{S}\right)(\lambda / \tau) \eta_{0}$. Note that this expression is a function of $\lambda / \tau$, like interface coupling.

Figure 2-9: Differential Acceleration

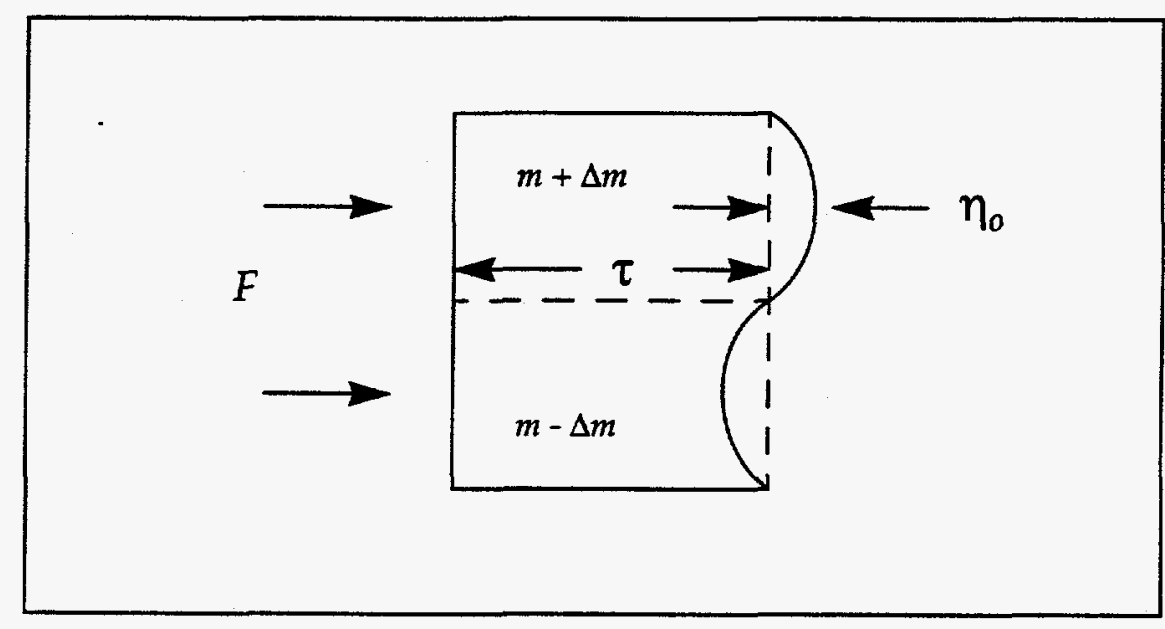




\section{Experiment}

A more in-depth discussion of the experimental procedure than found in the introduction is in order. A good understanding of the experimental procedure will allow the reader to evaluate the data with greater clarity, and repeat the experiments if necessary. In addition, experimental procedure is a type of technology. A successful procedure is not always obvious, and any information gained that could help other physicists in performing experiments is always of value. The experimental procedure used in the feed-out shots was based on previous successful experiments performed by other physicists found in the literature. A description of this previous work is documented in section 3.1. Section 3.2 describes the backlighter selection process, an important aspect of the experimental design. Section 3.3 reviews target fabrication procedures, whereas section 3.4 discusses target metrology. Fielding and diagnostics are covered in section 3.5.

\subsection{Related Experimental Work by Others}

By taking advantage of the experience of previous experimentalists, the feed-out campaign saved a great deal of time. Questions such as how much laser energy should be used on a Fe backlighter can only be determined by trial and error. The two sets of experiments described here had the configuration shown in Figure 1-5, with the exception that the perturbations were on the hot side, toward the hohlraum instead of on the cold side. Each experiment observed perturbation 
growth from the Rayleigh-Taylor instability on planar foils and was performed at the NOVA laser facility. Schappert conducted experiments with copper [Schappert] whereas Remington's foils were plastic, $\mathrm{CH}(\mathrm{Br})$, and fluorosilicone, $\mathrm{FS}$ [Remington 1992, 1993]. Both used $527 \mathrm{~nm}$ green light for the backlighters and 351 nm blue light for the hohlraums.

Schappert performed experiments designed by Hollowell. The package was a $16 \mu \mathrm{m}$ thick copper foil with a single mode, two-dimensional perturbation. Amplitudes of $0.4-0.5 \mu \mathrm{m}$ and wavelengths of 45 and $80 \mu \mathrm{m}$ were shot. Growth from the Rayleigh-Taylor instability was recorded using a microchannel plate and gated X-ray imager, which was believed to have a resolution less than $5 \mu \mathrm{m}$. They had difficulty seeing perturbations below $13 \mu \mathrm{m}$ in wavelength. Side views were obtained with a streak camera, which had sub-picosecond temporal and less than $5 \mu \mathrm{m}$ spatial resolution. The laser pulse shape was PS- 26 . The backlighter material and filter were both iron, which produced a very non-Planckian spectrum. The backlighter had intense lines at 6.7 and, to a lesser extent, $6.9 \mathrm{keV}$. The iron filter removed H-like lines, but allowed the He-like lines to pass through. Data was taken for $4 \mathrm{~ns}$ after the laser was fired. A strong second harmonic component was observed late in time.

The packages were modeled by Hollowell using 1-D LASNEX with the Takabe formula, Eq. (21), 2-D LASNEX, and 2-D RAGE. RAGE is a radiationhydrodynamics code with single group radiation and an adaptive square mesh [Gittings, Byrne]. A non-Planckian source including the gold M-band radiation 
was used. There was difficulty obtaining a good fit with the 1-D calculations, but the 2-D calculations matched the experimental growth rate reasonably well. RAGE 's square zoning simulated the perturbation in a stair-step fashion. At the beginning of the drive pulse, Hollowell found a jet of material emerging from each individual zone in the perturbation. The mode comprised of these jets appeared to couple with itself, producing a large second harmonic and matching the experimental results [Hollowell]. Although the jets were a computational artifact, something similar could have occurred in the experiment. The perturbation was machined into the copper in small steps of a similar size and shape as the square zones in RAGE. This high order perturbation could have coupled into the second as suggested by the code. Schappert hypothesized that the large second harmonic could also be a diagnostic artifact. Either insufficient camera resolution or an incorrect modulation transfer function could produce a spurious second harmonic, or portion of it. In late times, RAGE predicted a larger perturbation amplitude than observed. This discrepancy could have been due to the spikes being tilted at an angle to the camera.

Remington's experiments generated the hohlraum drive with a $3.2 \mathrm{~ns}$ shaped pulse and eight $2.1 \mathrm{~kJ}$ beams. The backlighter was a disk of $\mathrm{Mo}, \mathrm{Rh}, \mathrm{Sc}$, or Fe. It was illuminated with a ninth NOVA beam with a wavelength, shape, and energy of $0.53 \mu \mathrm{m}, 5.0 \mathrm{~ns}$ square, and $2.5 \mathrm{~kJ}$ respectively. There was concern that the backlighter structure might interfere with the experiment, so a random phase plate was used to smooth the beam's image on the disk. Remington used both 
face and side-on radiography. Perturbation images with two spatial dimensions were obtained in face-on radiography using a gated X-ray pinhole camera. For side-on images, either a streaked $22 \mathrm{X}$ Woelter $\mathrm{X}$-ray microscope, or a 20X magnification streaked-slit imager were used. Side-on diagnostics provided images with oné spatial and one temporal dimension. The side-on view was important to verify foil acceleration, and face-on was used to observe the growth of instabilities.

Remington's experiments included single mode, two-mode, and multimode initial perturbations. For the single mode, a $100 \mu \mathrm{m}$ wavelength initial perturbation with a $4.6 \mu \mathrm{m}$ amplitude was placed on the foil. The single-mode evolved into the nonlinear bubble and spike formation after $4.4 \mathrm{~ns}$, with the bubble growth asymptotically approaching that due to terminal velocity, and spike growth approaching that due to free-fall. There was a slight indication of foil bowing due to nonuniform drive from the hohlraum. For the two-mode case, wavelengths of 50 and $75 \mu \mathrm{m}$ were used, and the coupled components of 30 and $150 \mu \mathrm{m}$ were observed. The rough foil with random perturbations had a typical RMS deviation from the average of $1.7 \mu \mathrm{m}$ whereas a smoother random foil showed only $0.1 \mu \mathrm{m}$. The rough surface evolved after $4 \mathrm{~ns}$ into large, hexagonal bubbles of about $100 \mu \mathrm{m}$ in transverse size, while the smooth foil showed no obvious perturbation growth.

Weber obtained good agreement between Remington's results and computational modeling using a time-dependent drive spectrum [Weber]. NonLTE effects did not appear to be important. 


\subsection{Backlighter Selection}

An important part of the experimental design process was to select an appropriate backlighter for each package. An unsuitable backlighter would mean low quality data, or no data at all. The first step to good data involves the creation of a monoenergetic source, which is desirable to simplify the data analysis and prediction, as one did not then have to be concerned with a time-dependent spectrum and different opacities for each component of that spectrum. Due to the highly ionized nature of the backlighter plasma, the majority of ions are stripped to the He-like state. The He- $\alpha$ transition was the line used in these experiments for backlighting and occurs in the singlet state of the He-like ions. Because the $2^{1} \mathrm{~S}$ state of the singlet is metastable, the $\mathrm{He}-\alpha$ is a single line from the $2^{1} \mathrm{P}$ to the $1^{1} \mathrm{~S}$ state. A particularly convenient aspect of the He-like transition energies is that they usually occur just before the K edge of the material. A cold filter of the same material as the backlighter will then filter energies above and below the He-like transitions, producing an approximately monoenergetic source. The ICF experimentalist selects a backlighter material by looking up the $\mathrm{He}-\alpha$ transition energies of the elements. The element with the transition energy closest to the desirable energy for the experiment is then made into a backlighter disc.

There is a limited range of energies in acceptable backlighters. For high energies, above $\sim 9 \mathrm{keV}$, the opacity is very low, and the $\mathrm{X}$-rays no longer interact with the pinhole substrate in the X-ray camera strongly. The pinhole produces a 
fuzzy image, and the resolution decreases. Below about $3 \mathrm{keV}$ the spectrum becomes less monoenergetic. Another problem with using energies below $2 \mathrm{keV}$ is that the package and target themselves are thermally radiating, which could lead to a confusing signal. For these reasons, only backlighters between 3 and $9 \mathrm{keV}$ were considered.

An important concern in selecting a backlighter energy for face-on radiography is that the intensity be neither too large or too small. If the intensity reaching the film is too large, it will saturate, and if it is too small, no picture is recorded. Intensity is determined largely by material thickness and opacity. Xrays are attenuated as they pass through the package, according to

$$
T=\frac{I(z)}{I_{i}}=e^{-\mu \rho z},
$$

where $I_{i}$ is the initial intensity, $I(z)$ is the intensity a distance $z$ into the material, $\mu$ is the opacity and $\rho$ the density of the material. $T$ is the transmission ratio. The opacity is a function of X-ray energy and generally decreases exponentially with increasing energy. Thus, the higher the backlighter energy, the higher the transmitted intensity. The opacity can also be a strong function of material temperature for energies below about $3 \mathrm{keV}$. Above this energy, the cold, constant opacities were a reasonable assumption for the feed-out experiments. Intensity on the film increases with laser power on the backlighter and with the gain setting for the $\mathrm{X}$ ray camera. Intensity decreases with increasing backlighter energy. As the $Z$ number of the backlighter element increases, so does the energy of the He- $\alpha$ transition 
and the energy associated with the He ionization state of the element. For very high energy lines, the He ionization state energy may become much higher than the thermal plasma energy. The relatively large ionization energy results in fewer ions to create the line, and a weaker signal.

Unfortunately, the intensity of the backlighter is almost impossible to calculate due to the non-LTE nature of the plasma and great number of ion states. Likewise, the response of the film and the effect of adjusting the gain of the camera are unpredictable. Generally, the higher the energy, the greater the intensity seen by the camera due to increased transmission, up to about $8-9 \mathrm{keV}$. The initial density and thickness of the package were often used for approximate transmission calculations. Transmission ratios of $5-20 \%$ seemed acceptable for the $4-7 \mathrm{keV}$ backlighter energies used in the feed-out packages.

A second concern in the selection of backlighters for face-on radiography is that the intensity ratio of the perturbations be sufficiently large. As the backlighter $\mathrm{X}$-rays passed through the peaks of the perturbations, they were attenuated more than those passing through the troughs. The cameras were capable of observing a ratio of these intensities of about 0.9 , but generally lower intensity ratios were more desirable. If Zval is the package thickness in the valleys, while $Z p k$ is the thickness through the peaks, then the intensity ratio, $R$, is given by 


$$
R=\frac{I_{o} e^{-\mu \rho Z p k}}{I_{o} e^{-\mu \rho Z v a l}}=e^{-\mu \rho \Delta z} \quad \Delta z=Z p k-Z v a l .
$$

For an approximation at time zero, this ratio could be calculated using the initial conditions of the package. A much better estimate was made by using the TDG code, a LASNEX postprocessor, which produced contour plots of normalized intensity as a function of time. TDG used temperature-dependent opacities and density profiles from LASNEX. It also modeled the finite resolution of the camera, which decreases the observed intensity ratio. The backlighter was modeled as a spatially and temporally uniform monoenergetic source. The peak-to-valley intensity ratio could thus be plotted for a package versus time, given a specified backlighter energy. Such a calculation was conducted before each backlighter and package combination were fielded to make certain the perturbations were visible.

The intensity ratio decreases with opacity, which decreases with increasing $\mathrm{X}$-ray energy. For a larger intensity ratio, one would thus go to lower energy backlighters. This experimental constraint is in competition with the desire for high intensity. There is usually a range of backlighter energies for which both of these constraints are satisfactorily met. However, one must be careful with material selection for the package. For a given package thickness, if the $\mu \rho$ product for the material were too low, to obtain a satisfactory intensity ratio, one might be forced to use a backlighter of such a low energy that there was almost no transmission. Likewise, if $\mu \rho$ were too high, to obtain adequate transmission, one would be forced to use an energy too high for a good intensity ratio. 
The above discussion has been in regard to face-on radiography. Sideon radiography, will now be discussed as it has a different set of considerations. After the shock hits the perturbations on the rear surface of the package, material begins to move into the vacuum away from the package. A density gradient is established from the center of the package into the vacuum. The perturbations are usually located somewhere in this density gradient. The average density, size of the gradient, and location and size of the perturbation all change with time.

There is always some optimal X-ray intensity for imaging the package. $\mathrm{X}$-rays passing through the low density or vacuum region will overexpose the film. X-rays passing through the center of the package will all be absorbed. Somewhere in between there will be a density contour that attenuates the backlighter line to just the optimal intensity for the camera. The hope is that this is the density contour containing the perturbation. As the target expands and density drops, the perturbation moves from high to low density contours. High energy backlighters penetrate higher density contours, and thus show earlier time history of the perturbation. Lower energy backlighters will follow the evolution of lower density contours, and see the perturbation at later times. Experimental uncertainties in such things as camera response and laser energy on the backlighter thus result in a diagnostic uncertainty as to which density contour is followed. This situation is unlike face-on radiography, for which experimental uncertainties determine if the package is observable.

Design of a side-on experiment is more difficult than face-on radiogra- 
phy, as TDG can only predict relative intensity ratios. TDG was used to determine if the perturbations would be obscured too much by finite camera resolution. From the face-on experiments, it was known that certain combinations of backlighter energy and package thickness produced acceptable $\mathrm{X}$-ray intensities. To find the density contour that the same energy backlighter would follow in a sideon shot, the intensity for sidelighting was equated to that for face-on, and solved for the side-on density contour, $\rho_{S}$. In the equation below, $z_{F}$ is the package thickness for face-on experiments, whereas $z_{S}$ is the thickness for side-on shots. As the package was $250 \mu \mathrm{m}$ thick from the side, but only $35-85 \mu \mathrm{m}$ when viewed from face-on, the sidelighting density was much lower than the initial density of the package

$$
\begin{gathered}
I_{i} e^{-\mu \rho S^{z} S}=I_{i} e^{-\mu \rho z_{F}} \\
\rho_{S}=\frac{\rho z_{F}}{z_{S}}
\end{gathered}
$$

\subsection{Target Fabrication}

The creation of targets meeting the specifications of the experimental design was important to success, but it was not always easy. Because of the small number of targets used in the experiments, they were all made by hand. To fabricate the aluminum packages, a cylindrical copper substrate approximately $1 \mathrm{~mm}$ in diameter was placed on a lathe and machined flat. The substrate was then placed in a vacuum chamber and aluminum was vapor-deposited on the copper, 
see Figure 3-1. To generate the vapor, a piece of aluminum was placed in an indentation in a copper block, which was biased $10 \mathrm{kV}$ positive with respect to an electrical wire inside the chamber. Electrons were ejected from the wire and impacted the copper block and aluminum plug, causing aluminum ions to be ejected from the surface. To keep the aluminum from melting, water was circulated through the copper block. The copper substrate was positioned directly above and close to the aluminum plug, so as the aluminum ions were ejected, they were deposited on the substrate.

After a sufficient amount of aluminum was deposited, the substrate was placed back on the lathe and the aluminum machined flat to a thickness corresponding to the peak of the perturbations. The lathe was then used to cut a perturbation in the aluminum. The blade was not sharp enough to cut an exactly sinusoidal $50 \mu \mathrm{m}$ wavelength perturbation, but it produced a good approxima-

Figure 3-1: Vapor Deposition of Al on Cu Substrate

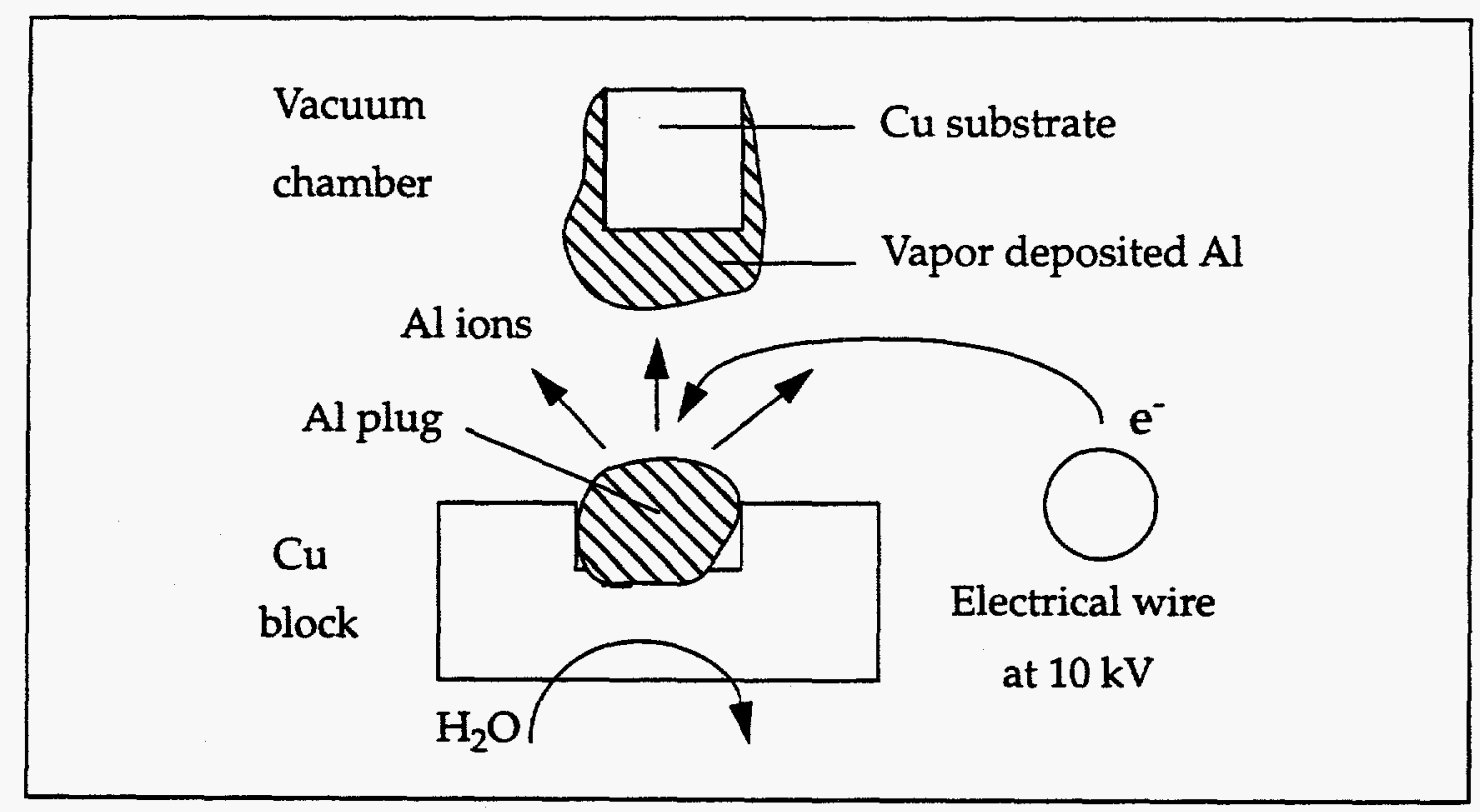


tion. The edges were then cut off with the lathe so the package could easily fit on the hohlraum. The copper was dissolved with nitric acid, which does not react with aluminum.

To fabricate packages of aluminum with beryllium on the perturbed side, the copper substrate was machined flat on the lathe, then the sinusoidal perturbation was cut into the copper. The substrate was placed in the vacuum chamber with a plug of beryllium in the indentation of the copper block. Instead of electrons, argon ions were accelerated through an electric field into the beryllium. The beryllium was thus deposited on the copper. The beryllium and substrate were then placed on the lathe again and the beryllium machined to $10 \mu \mathrm{m}$ thickness. This part of the procedure was difficult, as the beryllium would frequently flake off of the copper. To prevent this, it was important to have a very clean substrate before beryllium vapor deposition.

The beryllium and substrate were then placed back in the vacuum chamber and aluminum was vapor deposited on the beryllium. The aluminum was then machined flat to the desired thickness, and the copper dissolved with nitric acid.

Detailed drawings of the finished targets are shown in Figures 3-2 through 3-5B. The term LEH in these drawings refers to the "laser entrance holes" in the hohlraum. When the target is mounted in the NOVA target chamber, one LEH faces east, the other west. The terms east and west are therefore used to specify an individual LEH. The term SIM stands for "six inch manipulator." The SIM's 
are ports in the laser target chamber for diagnostics.

The targets were composed of a package, hohlraum, backlighter, alignment fiber, and radiation shields. The alignment fiber was used to align the targets in the NOVA target chamber. There were multiple radiation shields. One was a gold shield used for face-on packages that was placed on the side of the hohlraum with a hole cut just large enough to view the package. Without this shield, radiation passing from the inside of the hohlraum would degrade the data. Two other shields, the batwings, were glued to the ends of the hohlraum. As hot plasma squirted out the ends of the hohlraum, these shields kept the radiation from the plasma from overexposing the $\mathrm{X}$-ray film and ruining the data. The hohlraum, package, and shields were all glued together and to the end of a stalk coming up from a target stand. The backlighter was glued separately to another stalk attached to the same target stand. Small screws allowed fine adjustment of the

Figure 3-2: Hohlraum with Batwings

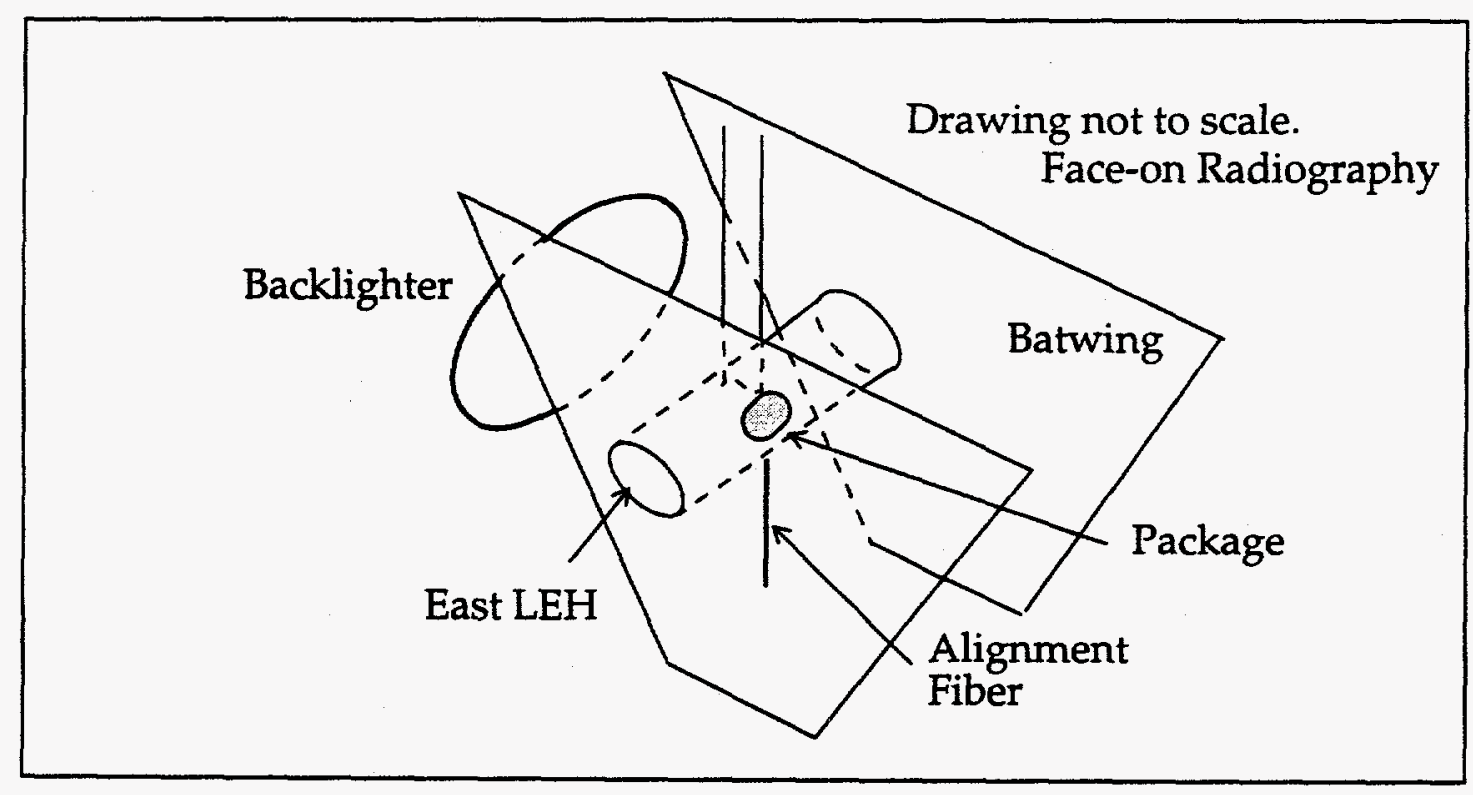


backlighter position.

Figure 3-3: Side View of Target Positioned in NOVA Target Chamber.

Face-on Radiography. Batwings are removed. Looking west into east LEH. All labeled dimensions drawn to scale 18:1. Scale I hohlraum.

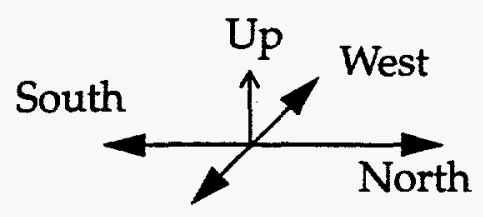

View from Backlighter disk seen on edge. East

- SIM 6 diagnostic
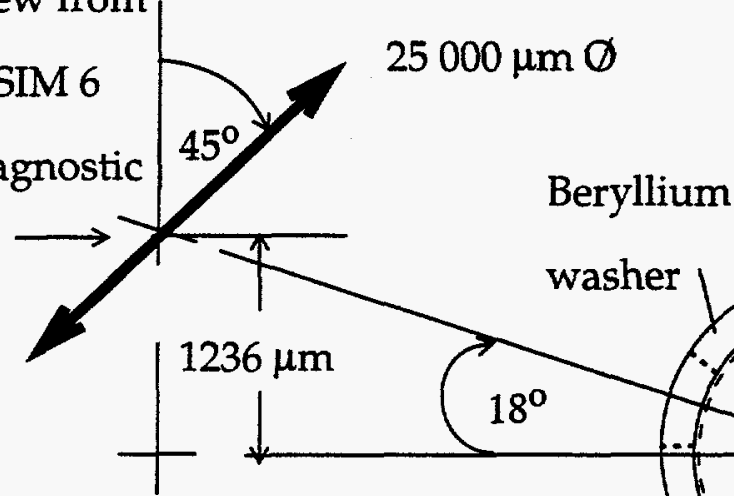

$1236 \mu \mathrm{m}$

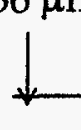
$3804 \mu \mathrm{m}$

Internal hohlraum radius $825 \mu \mathrm{m}$

LEH radius $600 \mu \mathrm{m}$

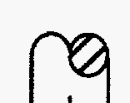

$25 \mu \mathrm{m} \mathrm{Au}$
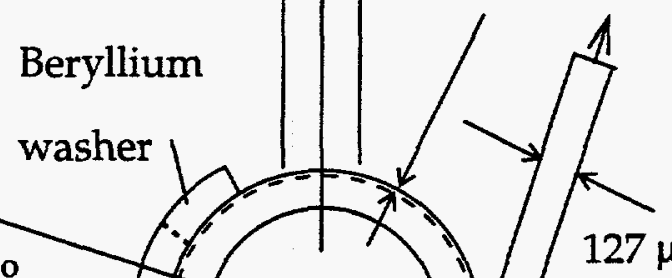

$18^{\circ}$
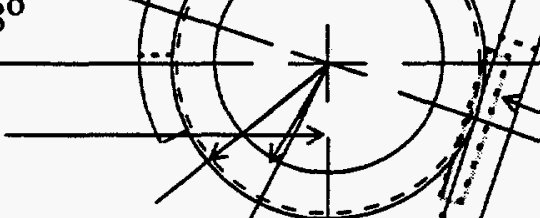

$127 \mu \mathrm{m}$

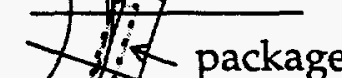

Au shielding 
Figure 3-4: Additional Views of Face-on Radiography Target

Front view of target from SIM 4 position with Au shield and batwings removed. SIM 4 is $18^{\circ}$ below the horizontal and to the north of the target.

All labeled dimensions drawn to scale 23:1.

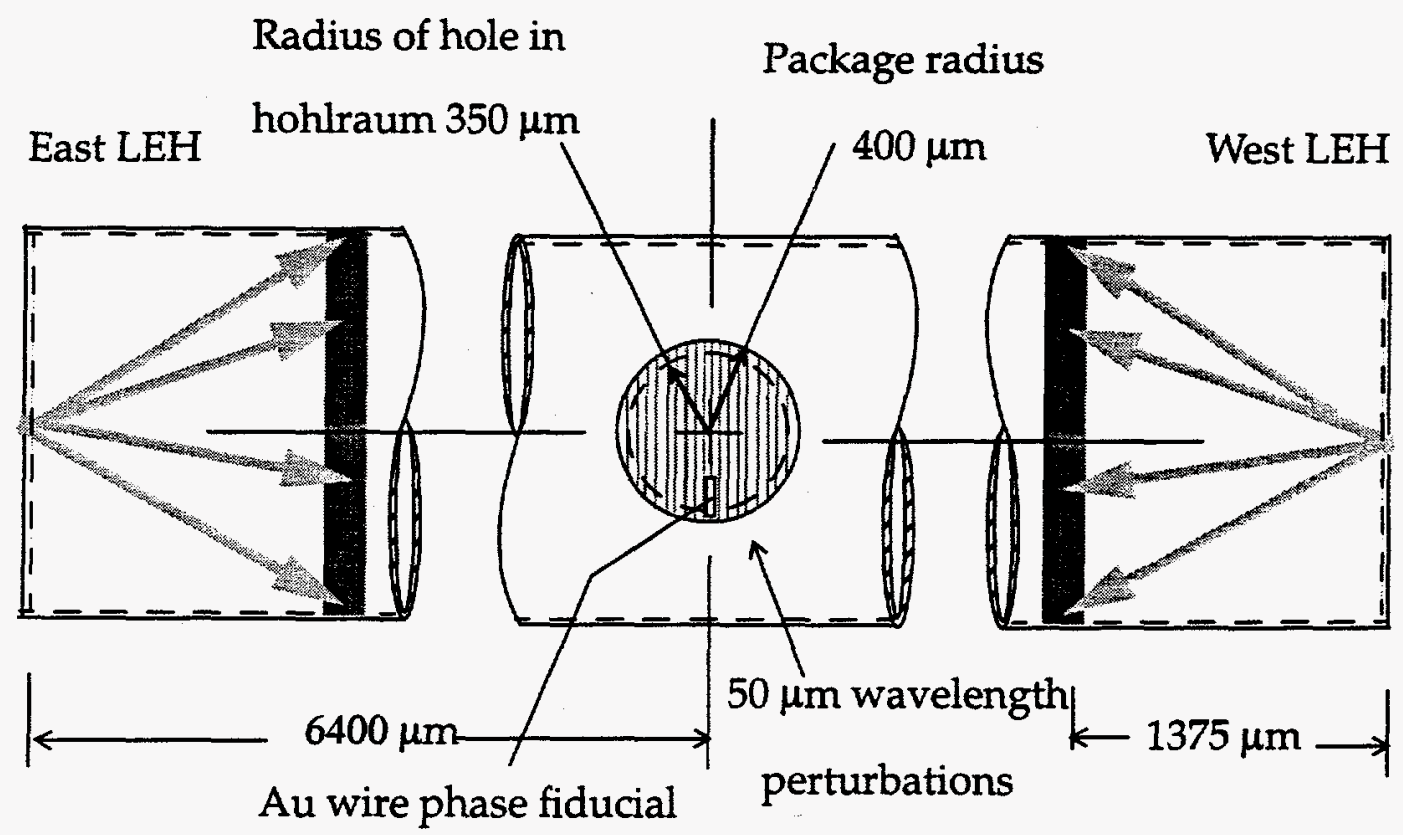

Back View of target looking toward SIM 4.

Scale 23:1

Be washer outer radius

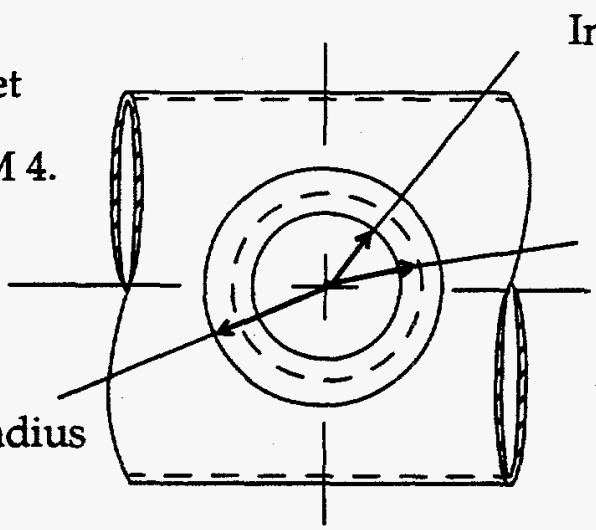

Inner radius of hole in

Be washer $313 \mu \mathrm{m}$.

Radius of hole in hohlraum on backlighter side, $400 \mu \mathrm{m}$. 
Figure 3-5A: Side-on Radiography

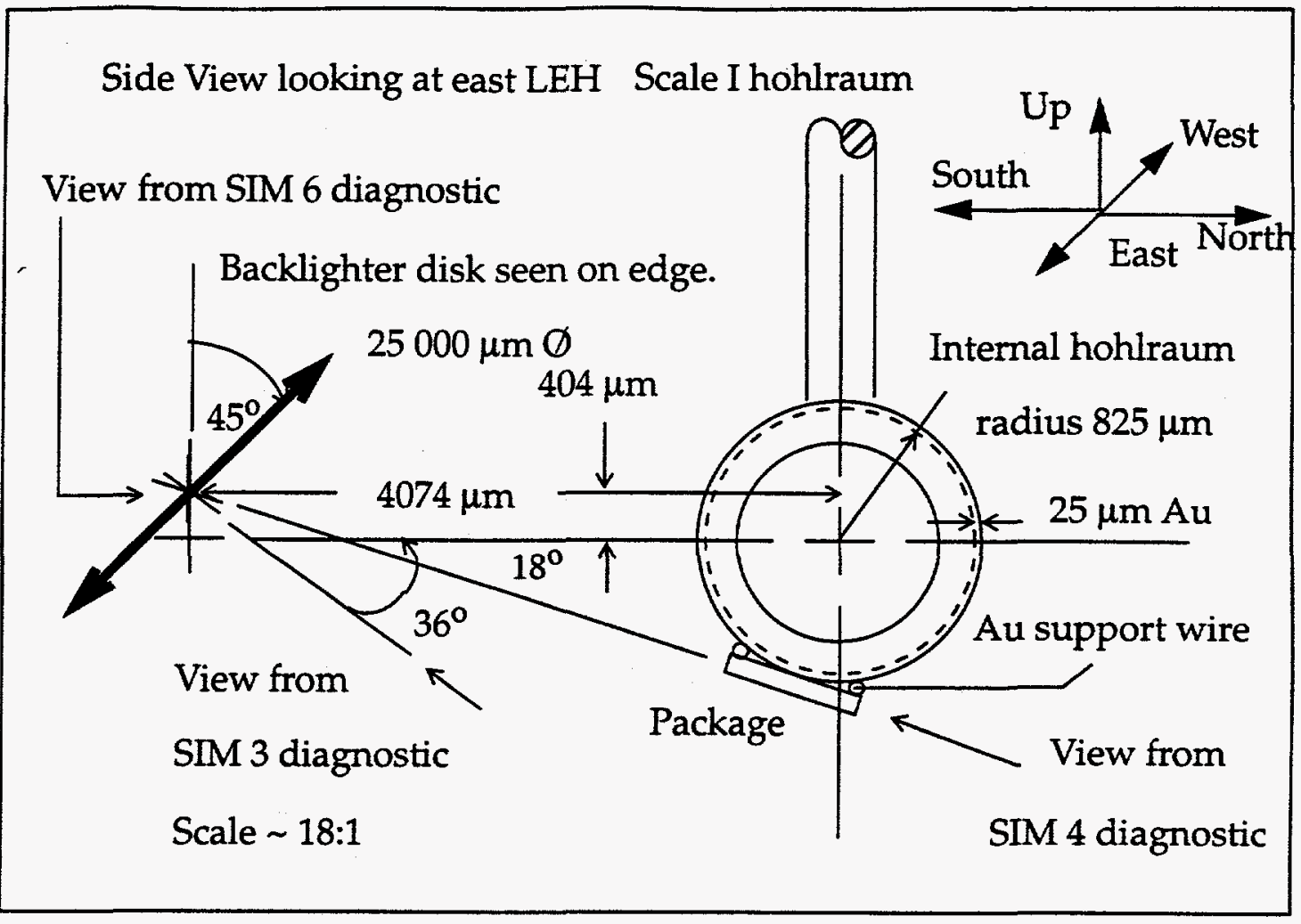


Figure 3-5B: Side-on Radiography

Front view from SIM 4 looking from the north $18^{\circ}$ below the horizontal.

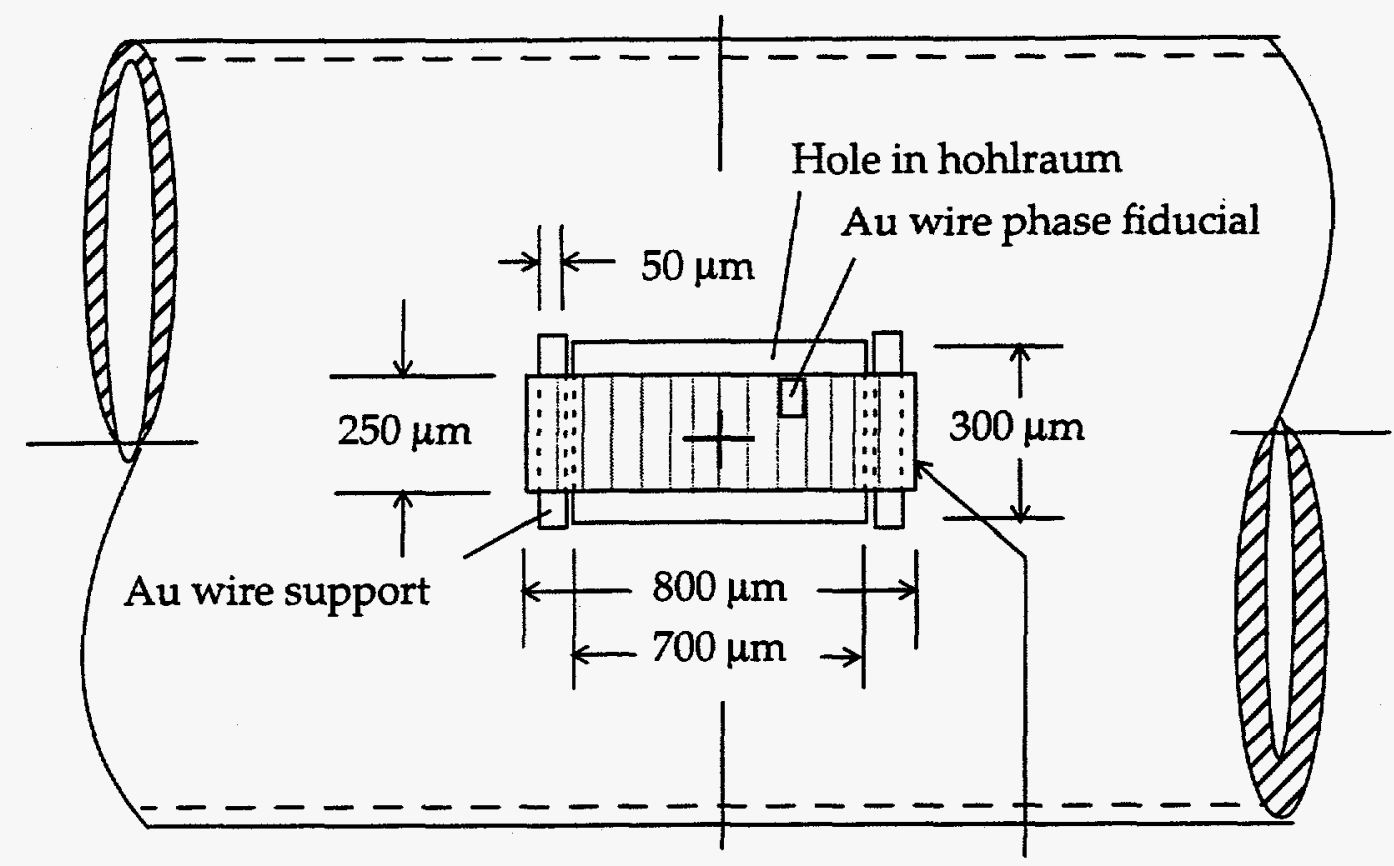

Scale $~ 50: 1$

Package with $50 \mu \mathrm{m}$ wavelength perturbations running perpendicular to cylindrical axis

\subsection{Target Metrology}

The day before the shots were taken, all targets were metrologized at Lawrence Livermore National Laboratory. In metrologizing the targets, one made certain that they were of a sufficient quality to render good data. Metrology allowed one to determine if the errors in angle and position of the fixed parts were acceptable, and to adjust the parts that were still movable to their optimal positions. Defects could sometimes be corrected on site, and pictures were always 
taken of the targets.

The metrology station consisted of a target manipulator accurate to a micron, computer software to control the manipulator, and a television screen connected to a camera and microscope for target viewing. Cross hairs on the television screen aided the work.

NOVA target chamber directions were used as reference positions on the metrology station. The target was initially positioned on the station with the operator facing the east LEH. The target could be moved up and down along the " $y$-axis", right and left along the " $x$-axis", and rotated at an angle $\alpha$ around the $y$ -axis. The first task was to adjust $\alpha$ such that the metrology station camera was looking in a line of sight parallel to the hohlraum axis. This was accomplished by first positioning the cross hairs on the far left hand side of the east LEH. The camera was then alternately focused on the east and west LEH's. The $x$ and $\alpha$ coordinates were iteratively adjusted until the cross hairs rested exactly on the far left hand side of both LEH's, indicating that the hohlraum was parallel with the line of sight. The center of the LEH was then found by measuring from one side to the next and dividing by two. LEH diameters were usually within about $10 \mu \mathrm{m}$ of the expected measure. By moving to the top or bottom edge of the hohlraum and alternately focusing on the east and west LEH's, one could obtain an estimate of how much the hohlraum axis deviated from the horizontal.

The cross hairs were next positioned at the center of the east LEH again, and the three coordinates were zeroed. The coordinates for the backlighter posi- 
tion were typed in. The backlighter was then moved to its optimal position by adjusting it until a small hole in the center of the disk was intersected by cross hairs on the television screen.

The target was rotated to the SIM 4 diagnostic view, showing the perturbed side of the package for face-on targets, and the side view for side-on targets. The center of the package was found and the $x$ coordinate recorded. The target was then moved along the $y$-axis until the alignment fiber was observed. The deviation of the fiber from package center was noted and used to better position the target in the laser chamber. It would have been preferable to measure deviation from hohlraum center, but this was not possible with the batwings attached.

Remembering the $x$ coordinate for package center, the target was rotated $180^{\circ}$, revealing the rear surface of the backlighter. The disk was then adjusted until the hole in its center was positioned at the $x$ coordinate for package center. Next the target was rotated to show the backlighter disk as seen by the two backlighter beams. If the beam paths were obscured by the batwings, the batwings were clipped. Lastly, the target was rotated to display the package edge on, and package thickness was estimated. Estimates of package thickness not be made in the targets with gold shielding, because the shielding obscured the package. 


\subsection{Target Fielding and Diagnostics}

The first step in fielding the targets was to provide NOVA technicians with a list of experimental details before each shot. These documents were termed set-up sheets, and provided information such as which diagnostics to use in which SIM, laser energy, diagnostic pointings, and so forth.

The X-ray camera looking at the package was placed in SIM 4, the streak camera looking at the backlighter in SIM 3, and the X-ray camera observing the backlighter in SIM 6, see Figure 3-6. Each port had small hand-turned cranks with which to adjust the viewing angle. To align the diagnostics, an alignment ball was placed in the chamber where the target would be. View ports, referred to as "target alignment viewers," or TAV's, were located directly opposite each of the diagnostic ports. Each TAV contained a telescope looking back along the line of sight of the opposing SIM. The shot physicist looked through a telescope while directing a technician in the adjustment of the diagnostic in the opposite SIM. Adjustments were made until the alignment ball was in the center of the snout of the diagnostic, as observed with the telescope.

The target was positioned in the chamber from the NOVA control room using the alignment fiber and the fibers' recorded deviation from package center measured in metrology. The two backlighter beams were turned on at very low power, and the beams adjusted so each laser spot was positioned correctly on the backlighter disk. Green light with a $527 \mathrm{~nm}$ wavelength was used for the two 
Figure 3-6: Section View of the NOVA Target Chamber

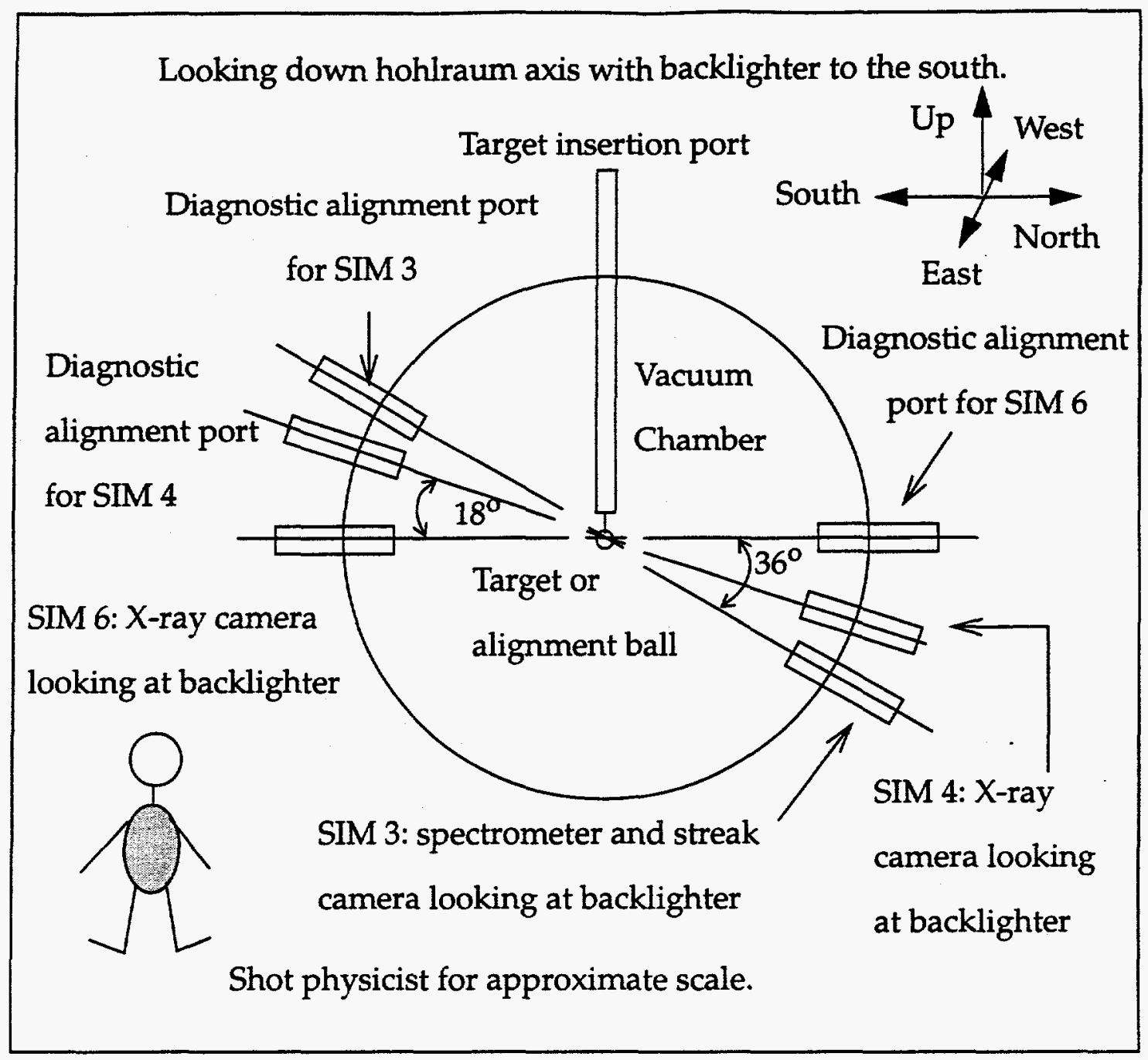

backlighter beams, whereas blue light at $351 \mathrm{~nm}$ was used in the hohlraum. Using two of NOVA's ten beams for backlighting caused the remaining eight beams to form an asymmetric ring of laser spots inside the hohlraum. This radiation asymmetry is not believed to have interfered with the experimental results.

Two types of X-ray cameras were used in the experiments, GXI's, or gated X-ray imagers, and FXI's, or fast X-ray imagers. There was no difference in the basic design of the cameras. The FXI was a commercially manufactured 
Figure 3-7: Operation of X-ray Camera

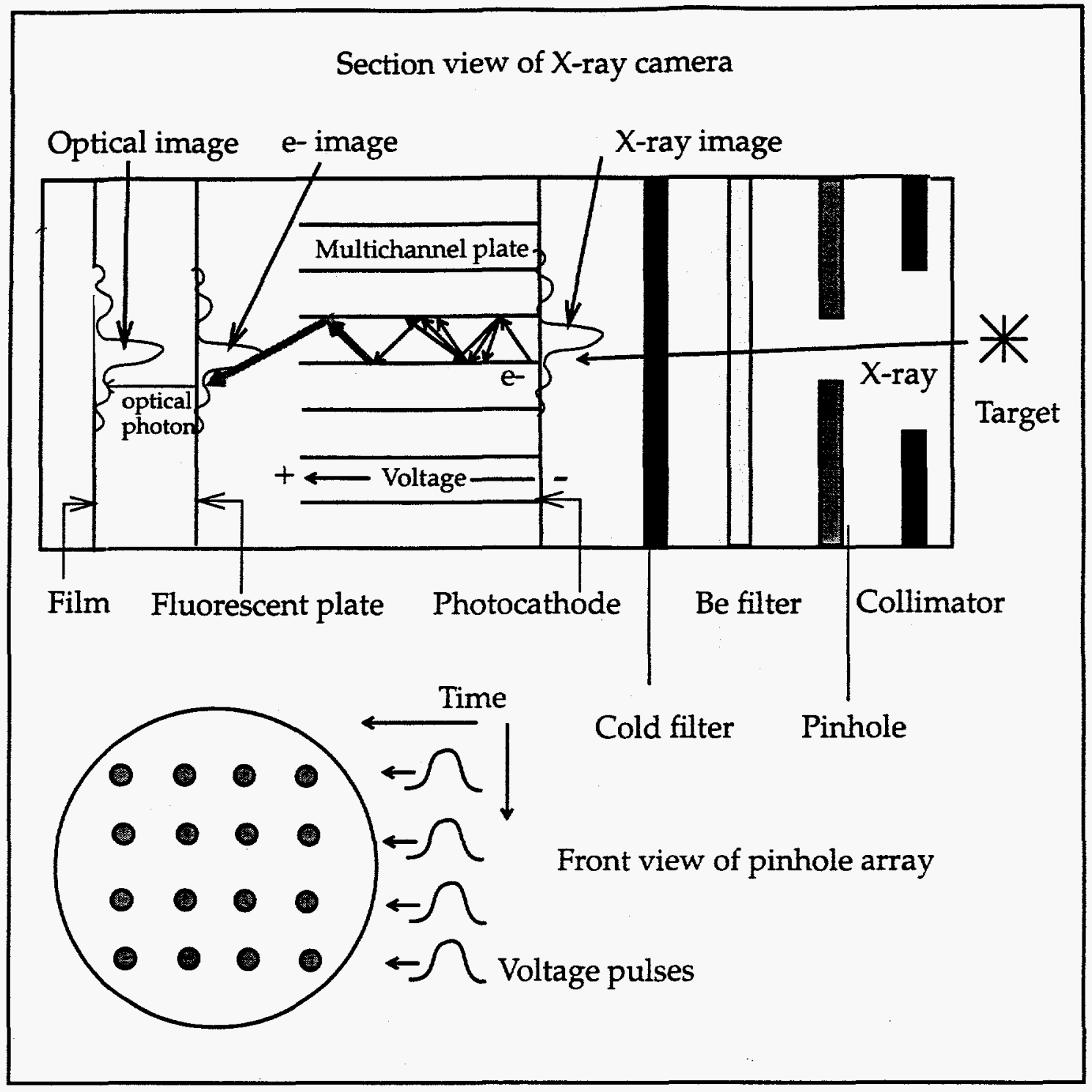

machine whereas the GXI was built at the laboratory.

Figure 3-7 shows camera operation. X-rays from the target passed through a collimator, pinhole array, beryllium filter, and a cold filter, before forming an image on the microchannel plate.

The collimator was composed of $250 \mu \mathrm{m}$ diameter holes, bored into a 75 
$\mu \mathrm{m}$ thick substrate. For some of the experiments, an additional collimator of 50 $\mu \mathrm{m}$ diameter holes was also used. The pinholes were $5-10 \mu \mathrm{m}$ in diameter and cut in a $75 \mu \mathrm{m}$ substrate. There were sixteen collimator holes and pinholes each in a four by four array. Each collimator hole was directly above a pinhole.

The beryllium filter protected the camera from debris as well as acting as a low energy X-ray filter for energies less than about one $\mathrm{keV}$, including the thermal hohlraum spectrum. The cold filter was of the same material as the backlighter and was referred to as cold because room temperature opacity tables could be used to predict its attenuation of the X-rays. The cold filter helped produce a very monoenergetic backlighter.

Behind each pinhole was a photocathode and microchannel plate, composed of millions of tiny tubes, arranged in a honeycomb fashion as viewed from the pinhole. After passing through the pinhole and filters, the $\mathrm{X}$-rays formed an image on the photocathode. An individual X-ray from the image would strike the surface of the photocathode, generating an electron by the photoelectric effect. The electron would then be accelerated down a tube in the microchannel plate, creating a cascade of electrons as it interacted with the wall. The process was similar to how a photomultiplier functions. At the end of the tube, the electron cascade struck a fluorescent material, emitting a flash of light, which was recorded by the film. The voltage in the microchannel plate could be adjusted, thus changing the gain of the camera to be more or less sensitive to X-rays.

Four pinholes in a line in the array were referred to as a strip. A timing 
delay could be set between the firing of the four strips, but no timing delay could be set between individual pinholes on the same strip. When a strip was fired, a voltage pulse would run down the strip, energizing each microchannel plate as it passed. Individual microchannel plates were only active during the time the voltage pulse was passing, and not after. The microchannel plates were active for about 80 ps each, during which time the image was formed on the film. Thus, if a process was shorter than $80 \mathrm{ps}$, there would be some blurring, but this was not a problem for the feed-out experiments. The total time for a pulse to run across a strip was about $250 \mathrm{ps,} \mathrm{so} \mathrm{there} \mathrm{was} \mathrm{some} \mathrm{temporal} \mathrm{overlap} \mathrm{in} \mathrm{the} \mathrm{images.}$

A single timing pulse from the NOVA control room was sent well in advance of the laser pulse to trigger the diagnostics. Each diagnostics defined the beginning of the experiment as the time at which it received the timing pulse. Just before the pulse from the control room reached a diagnostic, it entered a delay box for that diagnostic. The delay box added just the right amount of delay in order for the diagnostic to receive the timing pulse exactly at the beginning of the experiment. There was a different delay from the control room to each diagnostic due to differences such as cable length, and so each delay box had to be set to a different delay. In this way, all the diagnostics and the laser were fired simultaneously. The correct delays for each diagnostic were posted on the delay boxes. These delays were dialed in before each shot.

The initial timing pulse was broken into four pulses when it reached the camera, with each one being delayed somewhat with respect to the previous 
one. The first strip could be delayed for many nanoseconds from $t=0$. The succeeding three strips could be fired at a minimum of 250 ps apart, which resulted in a little overlap, or as much as 750 ps apart. Whenever two identical shots were taken, the strip timings were staggered so each shot covered the total temporal range of interest, against the possibility that one of the shots would not yield data.

The X-ray camera snouts were placed about $30 \mathrm{~mm}$ away from the target, providing a magnification of twelve times. For a pinhole camera, magnification is simply the ratio of the distance from the image to the pinhole, divided by the distance from the object to the pinhole.

The backlighter spectrum was recorded on each shot using a spectrometer and streak camera, see Figure 3-8. X-rays from the backlighter scattered off a crystal in the spectrometer and into the camera. Different frequencies were scattered at different angles, so the streak camera saw a spectrum. For each frequency there is an angle, its Bragg angle, for which $X$-rays scattered off two different crystal planes will constructively interfere. This angle is given by

$$
n \lambda=2 d \sin \vartheta,
$$

where $n$ is the order of reflection, and indicates whether the scattering planes are the first and second, $n=1$, first and third, $n=2$, and so forth; $d$ is the distance between planes, and $\theta$ is the scattering angle.

The spectrometer was aligned with the target using a screw that was on the snout and designated the centerline. The position of the crystal was adjusted such that when the He- $\alpha$ line from the backlighter scattered off the center of the 
Figure 3-8: Bragg Angle and Section View of Crystal Spectrometer

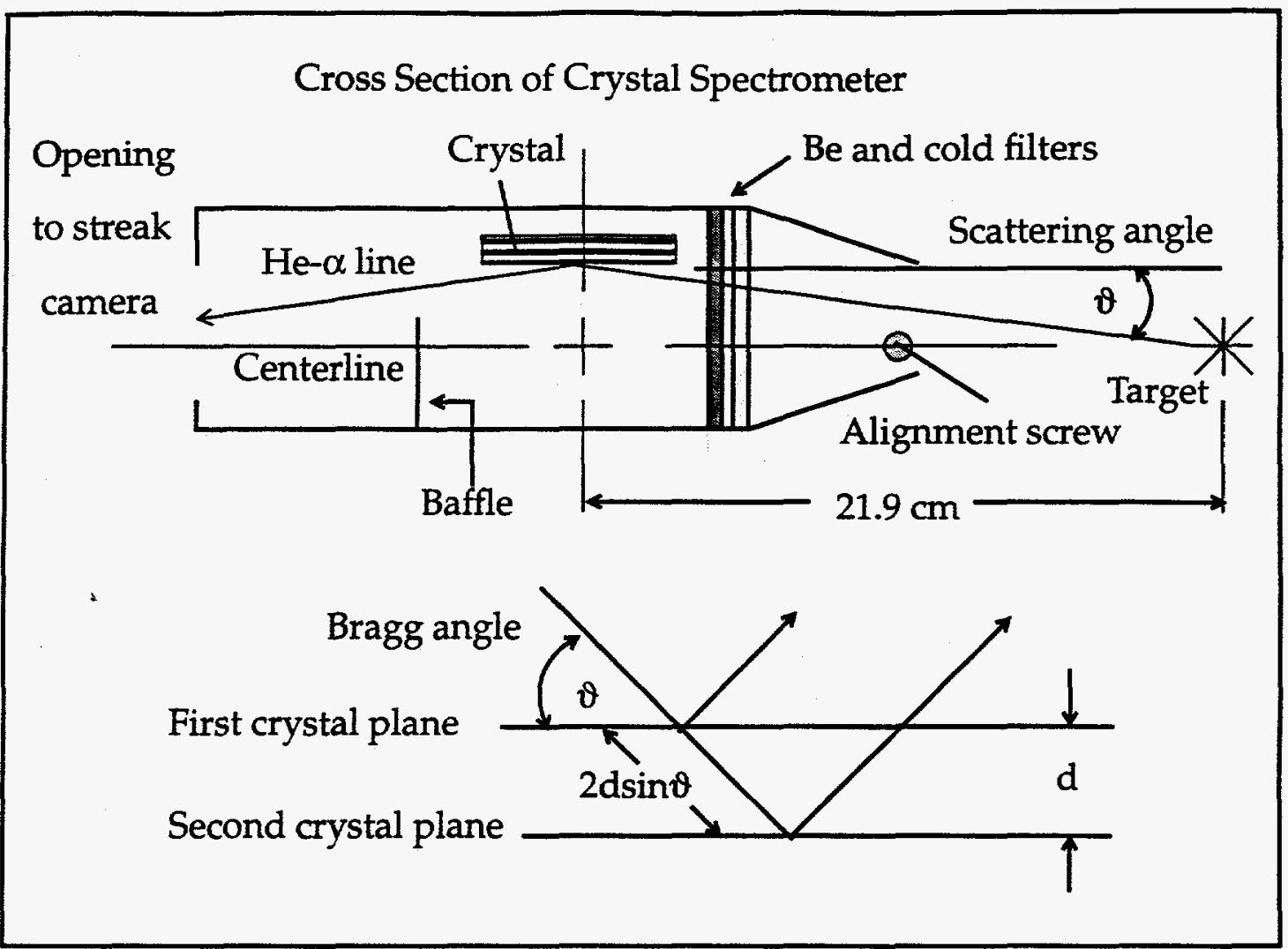

crystal, it was centered on the opening into the streak camera. First order reflection was always assumed. Two crystals were used in the experiments. For energies above $6 \mathrm{keV}$, a rubidium acid phthalate (RbAP) crystal was used, which had a $2 d$ spacing of $26.121 \AA$. For the lower energy backlighter, a pentaerythritol (PET) crystal with a $8.742 \AA 2 d$ spacing was used. Baffles in the spectrometer prevented the $\mathrm{X}$-rays from directly interacting with the streak camera, and a beryllium filter in the front prevented optical and lower frequency $X$-rays from interfering with the diagnostic as well as protecting the crystal from debris.

A streak camera is made to generate a picture with one dimension in 
space, and the other in time, see Figure 3-9. The temporal resolution is less than a picosecond, while the spatial resolution is on the order of $5 \mu \mathrm{m}$. X-rays from the spectrometer strike a photocathode, changing them into electrons. They drift through an electric field perpendicular to their motion and enter a microchannel plate. The electric field is generated by placing a voltage across two plates. The voltage is swept from a low to high value during the experiment and moves the electron image of the X-ray spectrum from the top to the bottom of the microchannel plate as it does so. The multichannel plate is similar to the one found in the GXI and FXI described above. The electrons emerge on a fluorescent plate, which converts them to optical photons, recorded on film.

There was some concern that the perturbation growth observed in the experiments was due to factors other than the machined perturbations. Target imperfections or drive asymmetry could be seeding the growth. To answer this

Figure 3-9: Streak Camera Operation

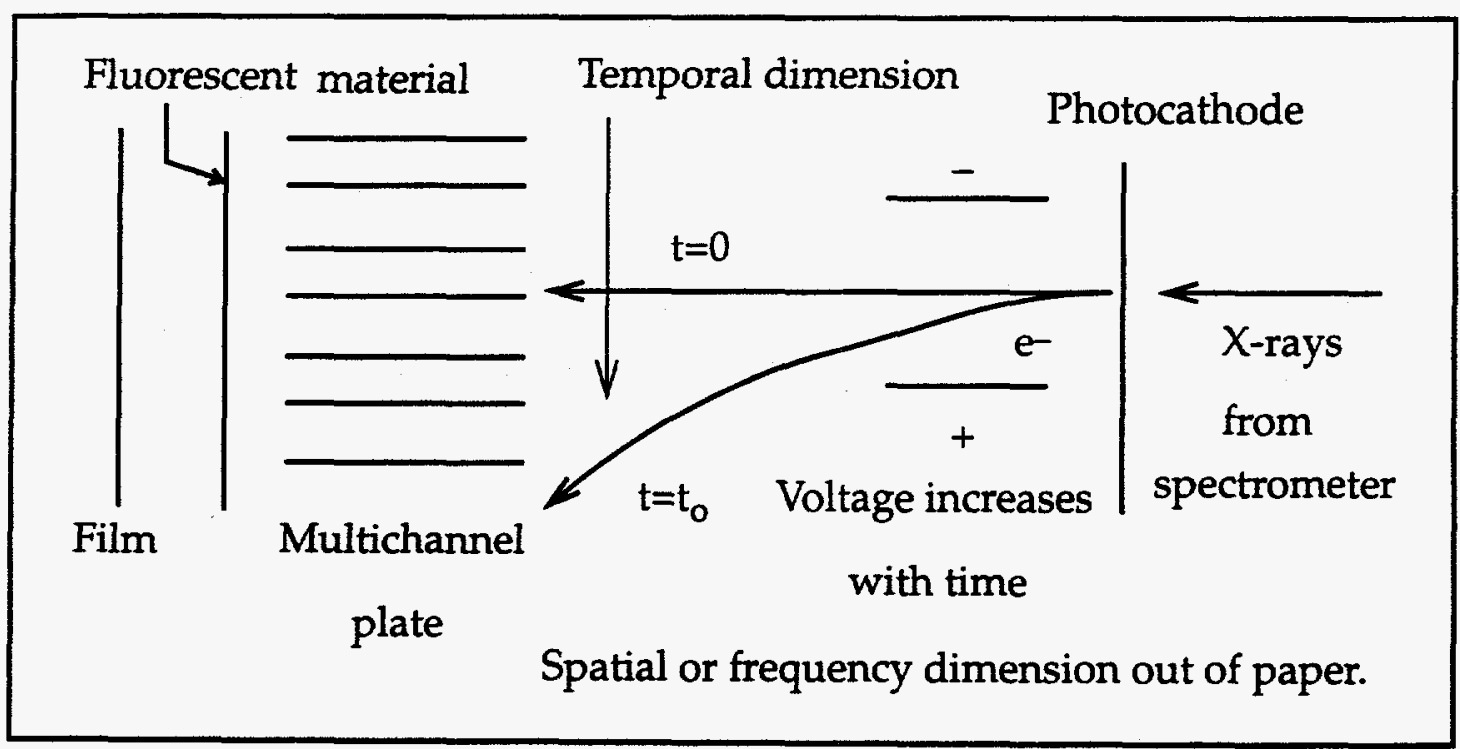


question, one shot was made with no initial perturbation. A Fourier analysis of the results was performed, and the part of the spectrum corresponding to the first harmonic in the perturbed packages observed. The first harmonic frequency showed a random fluctuation around a small initial value corresponding to a perturbation with a $\Delta \rho z$ of $5.59\left(10^{-4}\right) \pm 2.97\left(10^{-4}\right) \mathrm{g} / \mathrm{cm}^{2}$. The symbol $\Delta \rho z$ is a measure of the size of a perturbation and is defined as the difference between the integral of density along a line running through the perturbation peak and a line running through the perturbation valley,

$$
\Delta \rho z=\int_{\text {peak }} \rho(z) d z-\int_{\text {valley }} \rho(z) d z
$$

This shot also yielded an experimental error estimate of $\pm 0.3 \mathrm{~g} / \mathrm{cm}^{2}$ for the $\Delta \rho z$ of the packages with machined perturbations.

Some data analysis was required after the experiments were performed. The side-on data was only qualitatively compared to calculations, but the face-on Fourier spectrum was directly compared to computational results. To determine the spectrum from the data took several steps. The strips of film from the X-ray camera were digitized at NOVA and mailed to Los Alamos. The film did not darken linearly with light intensity, so the film response function had to be determined. In addition to the time history of a package, on each strip there was a "wedge" created by a uniform light source shining on the film through a filter. The filter was transparent at one end and gradually became completely opaque on the other. By performing a spline fit to the wedge, one could obtain the film 
response function.

An image of the package at a particular time was then selected. The average intensity of all the pixels around each individual pixel in the image was calculated. If the intensity of the central pixel deviated by more than a factor of 20 from the average, its intensity was set to the average, which helped to correct for defects in the film. The image was then corrected for film response, producing pixel intensities directly proportional to exposure.

Exposure was plotted along a line running perpendicular to the perturbations, producing what is called a lineout. The lineout had a long wavelength component due to variations in the backlighter intensity with position. The long wavelength was removed so the average value of the lineout function was zero. The natural log of the lineout was taken, and a Fourier transform was performed on the result. A cosine filter was used to eliminate high frequencies. There was usually some broadening of the harmonics around their central frequencies due to finite diagnostic resolution and the use of various filters, and possibly the physics. The amplitudes of these sidebands were added to that of the central harmonics. These values were divided by the modulation transfer function of the camera and compared to the calculations. 


\section{Computation with LASNEX}

LASNEX is a two-dimensional, cylindrical, Lagrangian radiationhydrodynamics code used primarily for modeling ICF plasmas [Zimmerman]. Runs are set up and controlled using a generator deck, a file created by the user with FORTRAN like commands for the main code. To run a planar calculation, such as for feed-out, one must place the package radially far away from the origin to minimize geometric cylindrical effects. The finite difference equations of hydrodynamics are solved directly, which is known as direct numerical simulation. Historically, this has not always been the case with hydrodynamics calculations, with perturbation growth being modeled by simple formulas on top of a zeroth-order hydrodynamics calculation. Partial pressures due to plasma components such as the ions, electrons, and photons are included in the hydrodynamics. The zoning is quadrilateral, with $\bar{z}$ and $\bar{r}$, components.

Because the code is Lagrangian, the mesh often becomes distorted. A remapping subroutine was used for the thin packages to allow the calculation to continue past mesh tangling. Comparisons of remapped calculations to pure Lagrangian for the thin packages showed little deviation up to mesh tangling, after which the remapper gave a more realistic answer.

Attempts were made to increase the speed of the calculation by "feathering" the zoning. In other words, increasing the thickness of each zone by a small factor, progressing from the initial perturbation to the ablation surface. These calculations produced incorrect results. It was found necessary to zone the ablation 
region as finely as the perturbation to obtain a correct answer.

In general, one needs finer radial zoning to resolve higher harmonics. For all but the thick packages, 45 radial zones were used in order to resolve down to the third harmonic. Convergence studies on the radial zoning indicated that this was sufficient. For the thick packages, 12 radial zones were used. Both Fourier analysis of these calculations and experimental data implied only a fundamental mode, so calculations with a better radial resolution were not pursued. Almost all of the calculations were run with a half-wavelength and reflective boundary conditions to save computer time. The zones were usually mass matched across the aluminum/beryllium interface to minimize the shock reflecting off of the mesh at this point. Some calculations were performed without radiation, where the shock was generated with a pressure source instead. These calculations had to have square root of density matching across the aluminum/ beryllium interface for the code to function properly.

The perturbation was generated in the mesh by slowly increasing the thickness of the zones as one moved from the bottom to the top of the package. Only the last $5 \mu \mathrm{m}$ of the mesh was perturbed. In hind-sight, this probably produced some computational error as the shock passed. In the thin target calculations, the remapper was turned on before shock arrival, so the shock interacted with a predominately square mesh.

The material surrounding the package was given a density several orders of magnitude below the package, to prevent it from interfering with the 
calculation or radiation delivery to the ablation surface. The low density of this material also contributed to mesh tangles. Edges of the mesh were placed far enough away from the package so shocks in the low density material did not strike the boundaries during the calculation, which increased computational speed.

Equations of state were calculated and tabulated by a group at Los Alamos specializing in the physics. The user specified the material in each region of the calculation and the corresponding equation of state to be used. LASNEX does not have strength of materials, and considers everything a fluid. Modeling of the packages in very early time going from solid to plasma, and in-between, is not done correctly.

LASNEX assumes thermal electrons to have a Maxwellian distribution. Transport of the electrons is accomplished with tensorial plasma conductivities in a magnetic field, modified by a flux limiter and variable degree of ionization.Thermal ions are heated by Coulomb collisions with the thermal electrons and by hydrodynamic compression. The Saha equation is used to determine the population of various charge species.

LASNEX is capable of modeling interaction of laser light with the hohlraum, the generation of X-rays, and interaction of the hohlraum spectrum with the capsule simultaneously. Modeling all of these processes simultaneously is referred to as an integrated calculation and is computationally intensive. For feedout package simulations, a much simpler approach was taken. A time dependent 
hohlraum spectrum was written in the generator deck that ran LASNEX. This spectrum was then used to drive the feed-out packages. For PS-35, only an experimentally determined hohlraum temperature as a function of time was available, so a Planckian source was used. Hohlraum spectrums normally contain a large gold M-band component of X-rays, greater than $2.5 \mathrm{keV}$, which is non-Planckian. The lack of data on the PS-35 M-band caused some error in the computational modeling of package preheat. The PS- 26 spectrum had been determined both by experiments and computation and included the M-band component.

Opacities, like the equation of state, were calculated and tabulated by a group at Los Alamos specializing in opacity physics. Although LASNEX has the capability to calculate its own opacities, these are believed to be inferior to the tabulated values. LASNEX opacities were only used to model the thick packages and seemed to generate a satisfactory result for these two cases. Tabulated opacities were used in all the other calculations.

To use the tables, the user creates an opacity file from them by specifying the desired mix of materials for each region and the binning. The radiation conservation equation is solved using a multi-group, flux-limited diffusion approach, with the groups being the user-specified opacity bins. Redistribution of photon energy due to Compton scattering is treated using a Fokker-Planck approximation.

To model feed-out packages, the binning ran from $30 \mathrm{eV}$ up to $50 \mathrm{keV}$. Below $30 \mathrm{eV}$, the frequency was close to the plasma frequency, and the radiation 
was totally absorbed. The hohlraum temperature was between 100-200 eV. Sufficiently refined binning below the $\mathrm{K}$ edge of aluminum, $1.56 \mathrm{keV}$, was important to correctly model the shocks and ablation region, while the bins above the $\mathrm{K}$ edge contributed mostly to preheat. Because of the low opacity of beryllium, it was not a consideration in binning. For the energies above the aluminum $\mathrm{K}$ edge, a standard diffusion model would be a poor representation, as the radiation has little interaction with the material and is very non-isotropic. The flux-limiting aspect of the LASNEX model should have compensated for this to some degree. Feed-out calculations were run assuming LTE, as a few non-LTE calculations did not reveal a substantial difference in the results. In hind-sight, the binning would have been better if consideration had been given to the radiation source binning in the LASNEX generator deck.

The first attempt at radiation binning involved the derivation of a simple equation describing the rate of energy exchange between the plasma and radiation field. Bins were then selected such that each represented the same rate of energy exchange on the average. Binning in this way did not succeed. The great majority of energy exchange was occurring close to the hohlraum temperature, so the binning was very tight around $200 \mathrm{eV}$, and unacceptably large over $600 \mathrm{eV}$. In response to this problem, logarithmic binning was adopted. Convergence studies were run for logarithmic binning above and below the aluminum $\mathrm{K}$ edge. The binning was found to have a strong effect on the shock strength. Acceptable convergence occurred with 20 bins below and 40 bins above the aluminum $\mathrm{K}$ edge. 


\section{Computational and Experimental Results}

Feed-out is a process by which a perturbation on the cold surface of a radiatively driven foil is hydrodynamically communicated to the ablation surface, seeds the Rayleigh-Taylor instability there and grows. The hydrodynamic signal that communicates the perturbation from the cold to the hot surface is referred to in this chapter as the "seed." As long as the foil is thick enough, the Rayleigh-Taylor and Richtmyer-Meshkov instabilities are independent, but as the radiation burns through the foil and the perturbations grow, they begin to interact. It is important to distinguish that this is a two step process. The perturbation feedsout, grows, and feeds-back in. The entire process is collectively and loosely called "feed-out" here.

The feed-out process is important in the study of ICF capsule physics because it connects the large internal perturbations on the DT ice with the strong, long-lived Rayleigh-Taylor instability on the ablation surface. A reduction of feedout is hypothesized to be responsible for the superiority of beryllium over plastic NIF capsules because of the additional thickness of the beryllium near the end of the pulse, and because of the larger density jump from the ice to the ablator. To study the effect thickness and density jump have on feed-out, calculations and experiments were performed and compared.

The reader is first presented with a brief review of how the data was obtained and considerations on how to best interpret the results presented here. Next, three fundamentally different feed-out situations in regard to thickness are 
discussed. The thick foil situation with little coupling between the cold and hot interfaces, the thin foil case with strong coupling, and the intermediate case which moves from weak to strong coupling during the shot. The first two cases are used to suggest that feed-out to the ablation surface is weakened as thickness increases. The latter case is instrumental in demonstrating that feed-in of the perturbation from the hot to the cold surface is inhibited by foil thickness.

There will then follow a discussion of computational and experimental evidence indicating that a density jump may have some effect on feed-out, but the effect is probably not large. Lastly, three interesting items will be reviewed which were learned during the course of the project, but may not be directly related to feed-out.

The majority of the data presented here is from face-on radiography and was Fourier analyzed to show modal structure. The Fourier amplitudes show how the perturbations grew and coupled. Exposure versus position data, see Figure 5-1, was first normalized for a specific time, then converted to attenuation versus position by taking the natural log of exposure. Attenuation is defined here as the integral of opacity, $\mu$, and density, $\rho$, along the path of the backlighter $X$-rays, $\int \mu \rho d z$. The attenuation versus position curve was Fourier transformed at different times in the experiment. Amplitudes of the various harmonics were then plotted against time. Because the data was normalized, the Fourier amplitudes are dimensionless. To compare with the data, LASNEX calculations were postprocessed using TDG to simulate diagnostic results. Both computational and experi- 
mental data are plotted in the figures that follow. In all the plots the open symbols with lines drawn through them, circles, bow ties, and triangles, are the TDG results and represent the first, second and third harmonics respectively. The closed symbols are experimental data. Error bars in the Fourier amplitude of all the data points are shown but are based solely on null shot data. There were many other factors contributing to experimental error including uncertainties in foil thickness and variations in the laser energy and pulse shape. Some of the experimental variations are recorded in the appendix. The reader should also keep in mind that there is an estimated 500 ps timing error on all points which is not shown to make the plots more readable. The noise level of each experiment is recorded on the graphs.

Interpretation of the data shown in the following figures must be made with care. Face-on data does not show the location of the perturbations, whether they are on the ablation surface, the cold surface, or are internal oscillations. Sometimes the results are a conglomeration of perturbations in all three locations. Modes that are in phase and of the same amplitude on both the ablation and cold surfaces are not observable, see Figure 5-1A. They produce a sinuous shape, which has an identical attenuation for X-rays passing through the trough or the peaks. The sinuous pattern occurs most frequently with modes that are coupling strongly across the interfaces and is thus more prevalent in the fundamental than in the harmonics due to the difference in wavelengths. In addition, the same perturbation with the same phase has a different Fourier transform phase depending 
Figure 5-1: Fourier Amplitudes and Surface Perturbations

\begin{tabular}{|c|c|} 
B) \\
Backlighter \\
B) \\
Backlighter \\
Fourier transform with \\
positive phase \\
Both perturbations have same phase and amplitude.
\end{tabular}

on whether it is on the hot or cold surface, see Figure 5-1B, because the phase of the attenuation curve changes.

The time history of the fundamental Fourier amplitude mode in the 86 $\mu \mathrm{m}$, thick aluminum package is shown in Figure 5-2. The data is from shots 1 and 6 which used PS-26, the 2.2 ns pulse (see the appendix for shot details). The fundamental has a non-zero value at the beginning of the experiment, indicating that the diagnostic could see the initial perturbation. At about $2 \mathrm{~ns}$, the shock arrived 


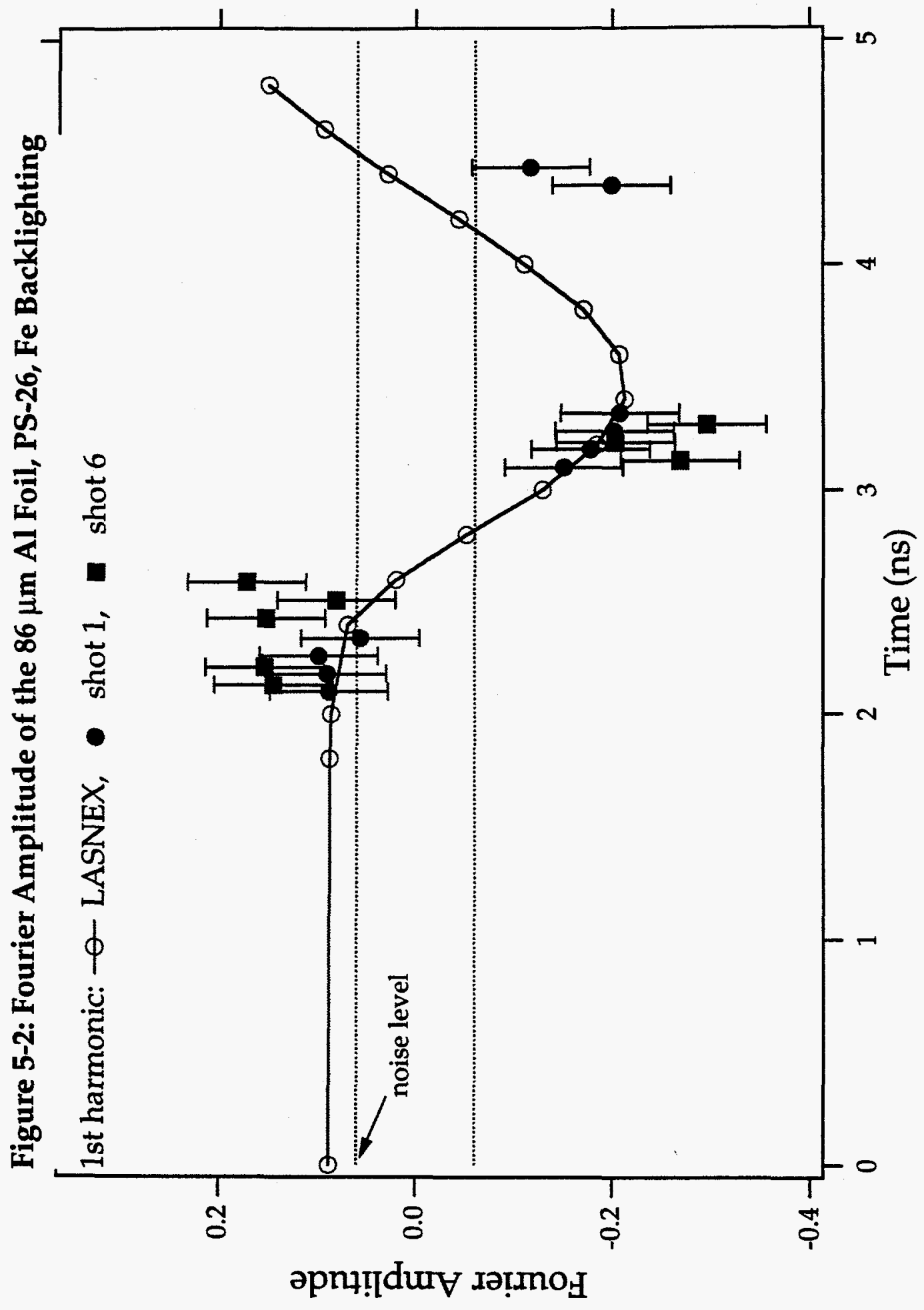


at the cold surface and induced a Richtmyer-Meshkov instability. The perturbation phase-inverted and grew. Although the laser pulse ended at $2.2 \mathrm{~ns}$, residual radiation from the hohlraum continued to accelerate the foil for perhaps another $600 \mathrm{ps}$. At $3.5 \mathrm{~ns}$, the Fourier amplitude of the fundamental began to increase and pháse-inverted a second time. Computational results indicate that this second phase-inversion was the result of the oscillation of a strong internal acoustic mode associated with the Richtmyer-Meshkov instability. The perturbation on the cold surface did not actually invert a second time.

The shock hit the cold surface about the time the pulse was turning off. As a result, the perturbation did not feed-out to the ablation surface in time for Rayleigh-Taylor growth. All of the activity observed in this foil is thus representative of the evolution of the seed for the Rayleigh-Taylor that feeds-out to the ablation surface. Only the fundamental is shown in Figure 5-2 because higher order harmonics did not appear in either the data or calculations. This would be consistent with only the Richtmyer-Meshkov instability, which produces harmonics slower than the Rayleigh-Taylor instability. In addition, side-on radiography showed growth of the cold surface perturbation, but a perfectly smooth ablation surface out to $4.5 \mathrm{~ns}$, see Figure 5-3, indicating there was no Rayleigh-Taylor growth. The side-on data also supports the supposition that there was very little coupling between the cold and hot surfaces due to the excessive thickness of the foil. Feed-out is probably more difficult in thicker foils. Figure 5-3 shows two strips from the framing camera for both the thick and thin foils, with four images 

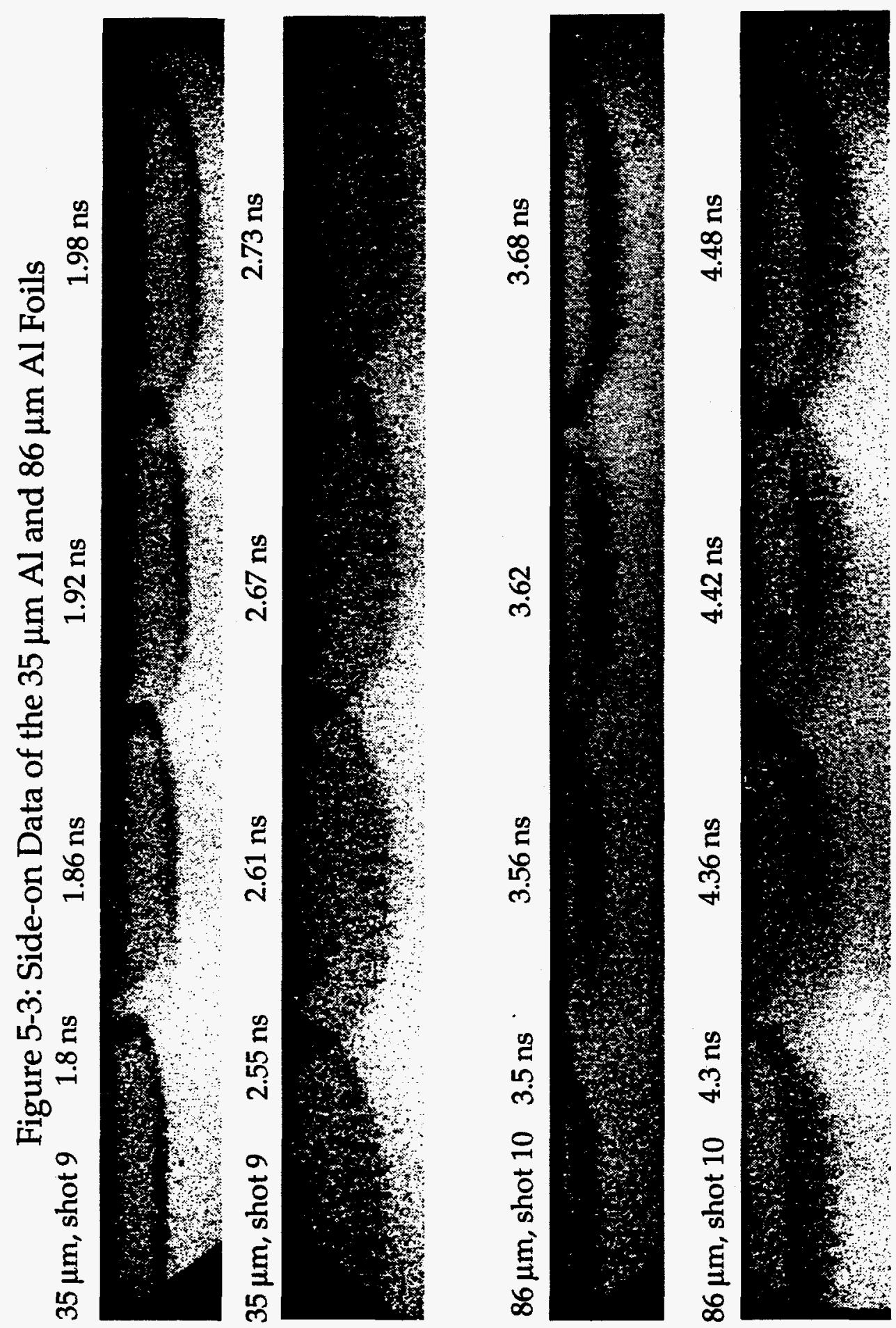
per strip. The thin dark area in the center of each image is the foil. Radiation comes from the dark area just above the foil, where the hohlraum is located.

Figure 5-4 shows the Fourier amplitudes from shot 3 , the $35 \mu \mathrm{m}$, thin aluminum package driven with PS-26. Because of the relative thinness of this foil in comparison to the wavelength, there was a very strong coupling between the hot and cold surfaces. The shock hit the cold surface perturbation around $1 \mathrm{~ns}$, and the perturbation began to phase-invert, producing a drop in the fundamental's amplitude. Before shock arrival, preheat created a small density gradient into the cold side vacuum, which may have partially stabilized the Richtmyer-Meshkov instability. By $1.4 \mathrm{~ns}$ a small perturbation appeared on the hot side of the package from feed-out and the package evolved rapidly into a sinuous shape. The sinuous pattern from feed-out may be easily observed in either the side-on data in Figure 5-3, or in the computational results in Figure 5-5. This was the first experimental confirmation of feed-out in radiatively driven foils. The sinuous shape reveals that the fed-out perturbation is in phase with the cold surface perturbation. This would be expected from instabilities strongly coupling across two interfaces.

The maximum density of the package dropped below the solid density of aluminum at $2 \mathrm{~ns}$, and the package began to burn through. About this time the Rayleigh-Taylor instability caused a large growth in the ablation surface perturbation and spawned second and third harmonics. The Fourier phase of the second harmonic was opposite that of the fundamental and the third harmonic. The Ray 


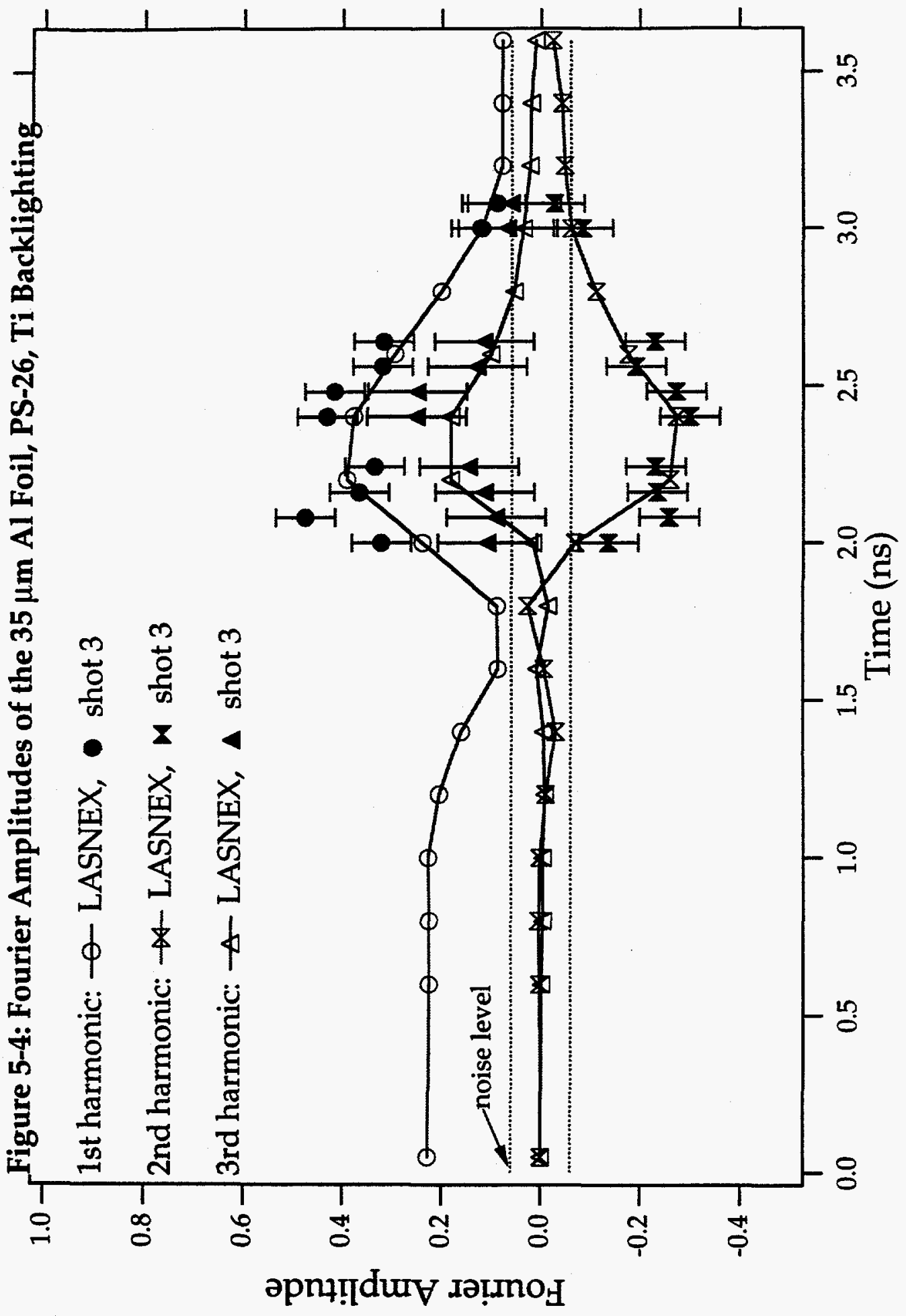




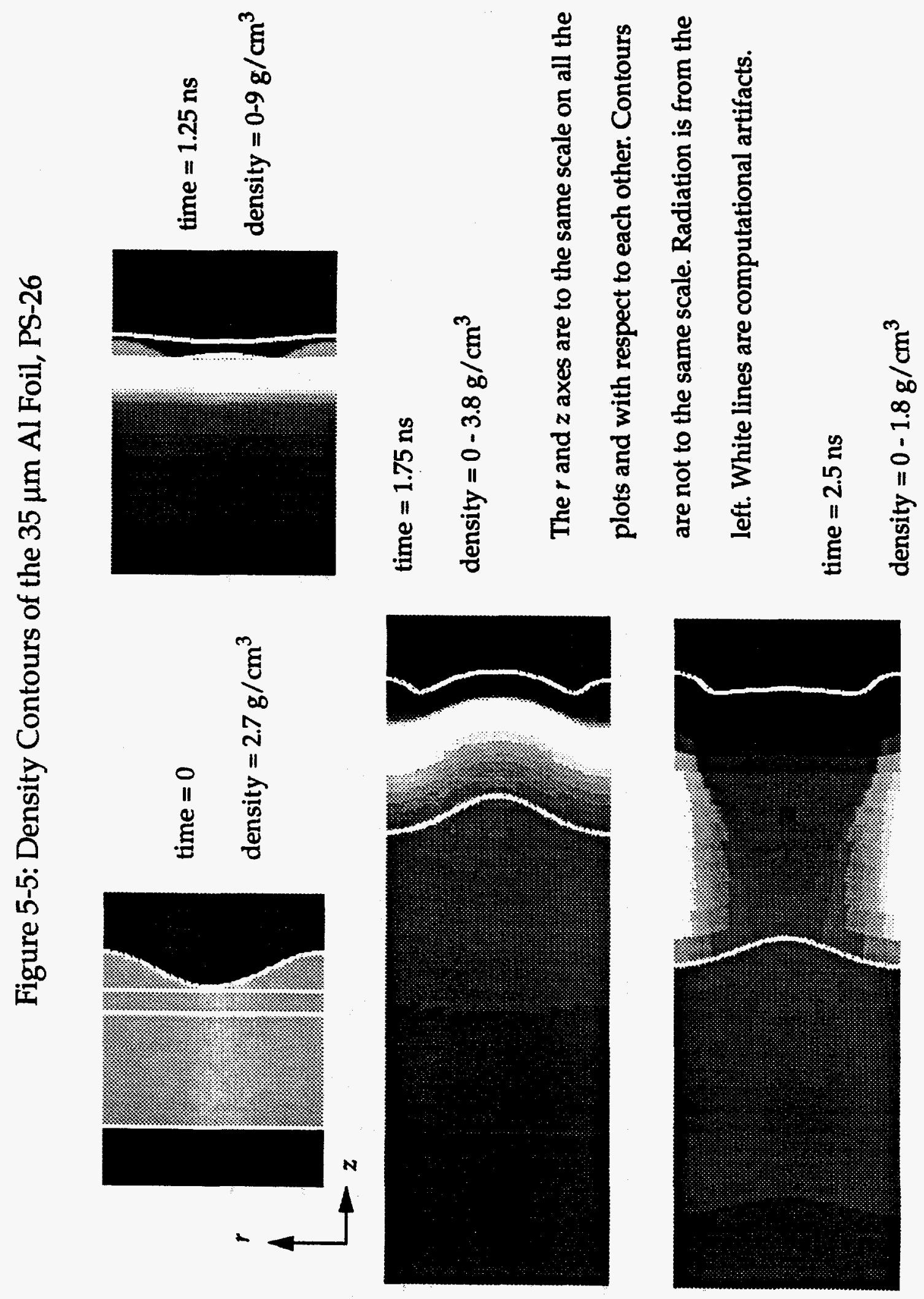


leigh-Taylor instability reversed the phase inversion of the fundamental and by 2 ns the fundamental's amplitude increased above the original amplitude. After 2.5 $\mathrm{ns}$, the package density continued to decrease as the foil broke apart, and the Fourier amplitudes decrease correspondingly. During late times, the foil evolved into a nón-linear bubble and spike configuration as shown in Figures 5-3 and 5-5. The bubbles have burned through in both figures.

From the computational results, face- and side-on data, it is clear that the thin foil's physics is fundamentally different from the thick foil due to the greater interface coupling and the drive pulse being on during feed-out. This difference underscores the importance of thickness and pulse length in the feed-out process.

Beryllium was placed on the cold surface of some of the foils in order to observe any density jump effect, but also to observe feed-out of the seed. The beryllium was transparent to the backlighter, so when the seed moved from the beryllium into the aluminum, it could be observed. If the amplitude of the seed was smaller at the aluminum/beryllium interface than at the cold surface of the pure aluminum foils, this would help confirm that feed-out was a dependent on thickness. The question of whether or not the feed-out seed decayed with distance from the cold surface was particularly interesting in light of the theoretical work discussed in chapter 2 predicting that the perturbation amplitude on a rarefaction wave was stable.

Face-on data from the thick aluminum/beryllium composite foil, shot 
2, is shown in Figure 5-6. Notice that the diagnostic cannot see the initial perturbation, which is in beryllium. All of the observed perturbation growth is internal to the foil and has feed-out from the beryllium to the aluminum. A comparison between Figures 5-2 and 5-6 shows little difference. The beryllium was only $10 \mu \mathrm{m}$ thick. If there was attenuation of the seed with distance from the cold surface, 10 $\mu \mathrm{m}$ might not have been large enough to observe the attenuation, so the result seems inconclusive.

A subtle aspect of the physics of the thick aluminum/beryllium foil was that the aluminum/beryllium interface could have become Rayleigh-Taylor unstable. This was an important concern in interpreting the data. If the interface was unstable, then perhaps the growth that was observed was due to the Rayleigh-Taylor instability and not due to the seed from feed-out. As long as the drive was on, the aluminum was pushing the beryllium and the interface was stable, but after the drive was off the foil began to expand. If the pressure dropped faster in the aluminum than the beryllium during the expansion phase, then a pressure gradient could form across the interface of the two fluids with an opposite slope to the density gradient. Density and pressure profiles with opposing slopes across the aluminum/beryllium interface would render the interface Rayleigh-Taylor unstable. The calculations indicated that this situation did not occur and that Figure 5-6 should only show feed-out into the aluminum. 


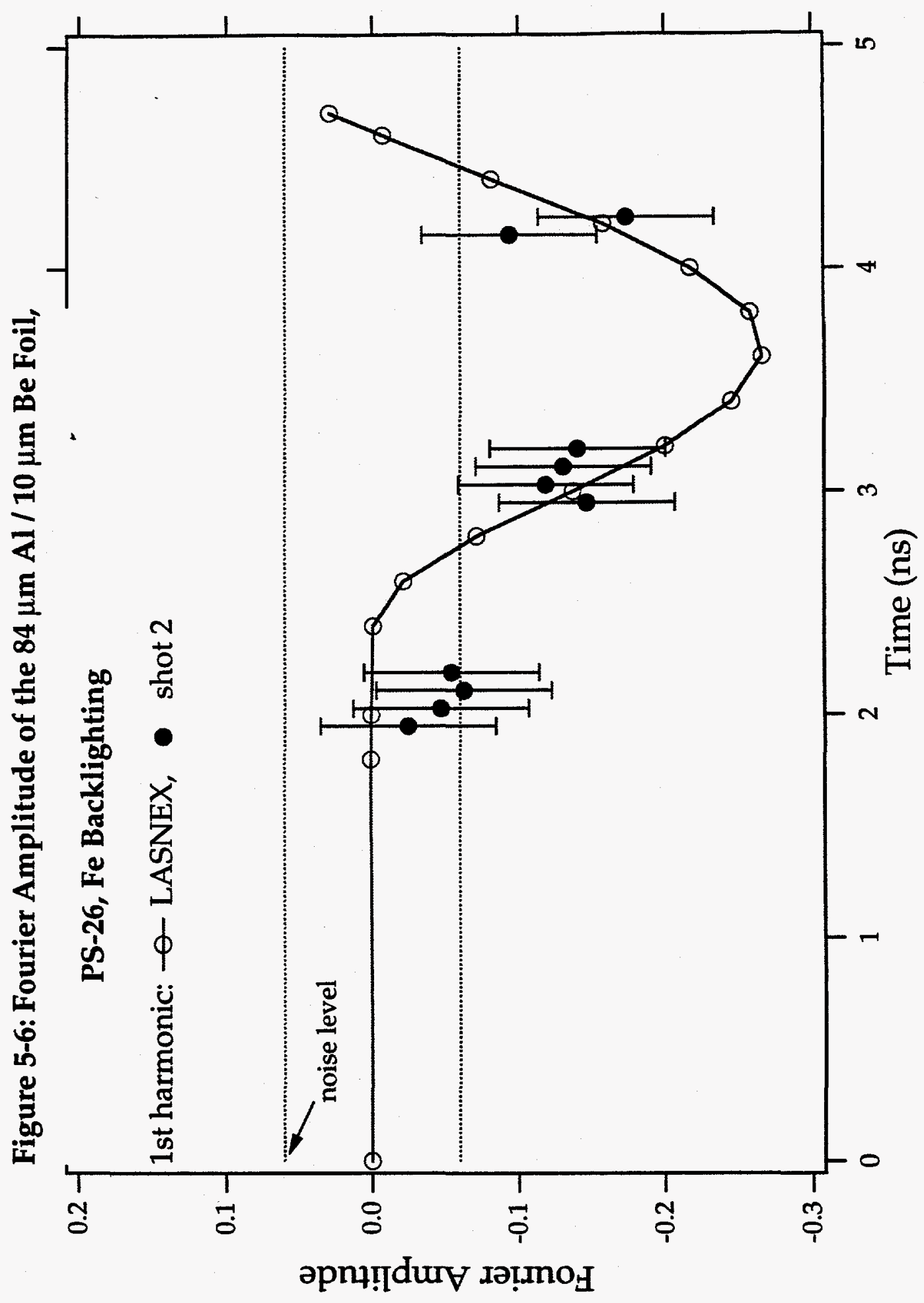


Figures 5-7 and 5-8 show the Fourier modes for the $32 \mu \mathrm{m}$ aluminum/ $10 \mu \mathrm{m}$ beryllium packages shot with PS-26. The former plot is for shot 4 with a titanium backlighter, while the later plot shows shots 18 and 19 with a scandium backlighter. There was no concern in these shots of an unstable aluminum/beryllium interface as the drive was on almost the whole time of interest.

Two prominent peaks in the fundamental mode are seen at $1.6 \mathrm{~ns}$ and $2.4 \mathrm{~ns}$. The second peak is probably Rayleigh-Taylor growth on the ablation surface. Its timing corresponds to Rayleigh-Taylor growth of the fundamental shown in Figure 5-4. The first, smaller peak is a mystery. Originally, the first peak was believed to be the feed-out seed entering the aluminum, but the peak is the wrong phase. In all the other experiments, both pure aluminum and composite, the initial growth and feed-out produces a negative Fourier amplitude phase. One would have expected a dip in the fundamental Fourier amplitude followed by a sharp rise from the Rayleigh-Taylor growth.

Another surprising and potentially important lesson was learned from the thin composite foils. The data did not reveal the presence of harmonics above the noise level in these packages. Calculations only predicted a second harmonic amplitude slightly above the noise level. One might therefore suspect that the second and third harmonics observed in the $35 \mu \mathrm{m}$ pure aluminum packages were growing on the cold surface, but not on the hot surface. The lack of harmonics in the thick packages indicates that the Richtmyer-Meshkov instability was not producing the harmonics. Perhaps the higher harmonics observed in the $35 \mu \mathrm{m}$ 


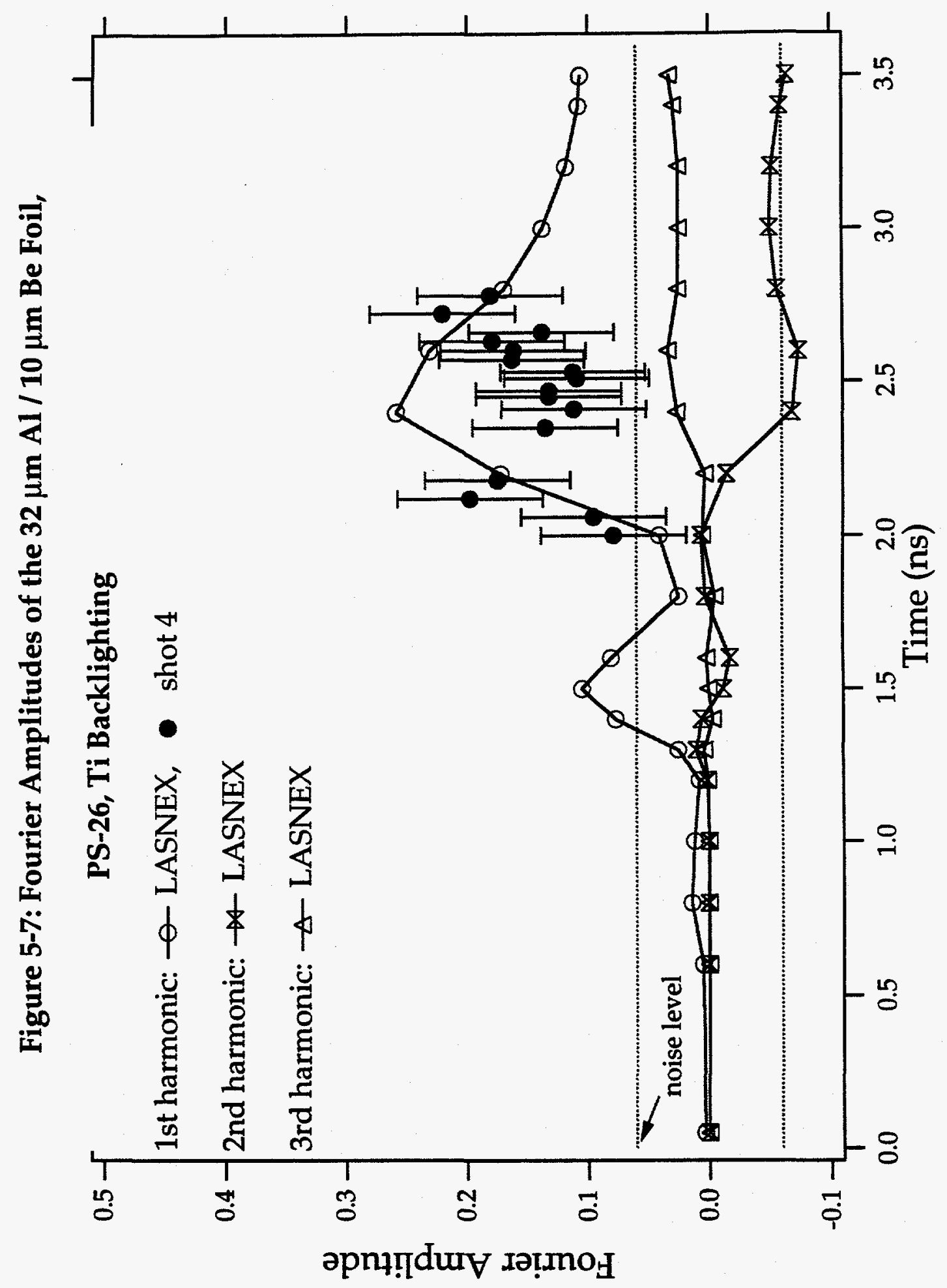


Figure 5-8: Fourier Amplitudes of the $32 \mu \mathrm{m} \mathrm{Al} \mathrm{/} 10 \mu \mathrm{m}$ Be Foil,

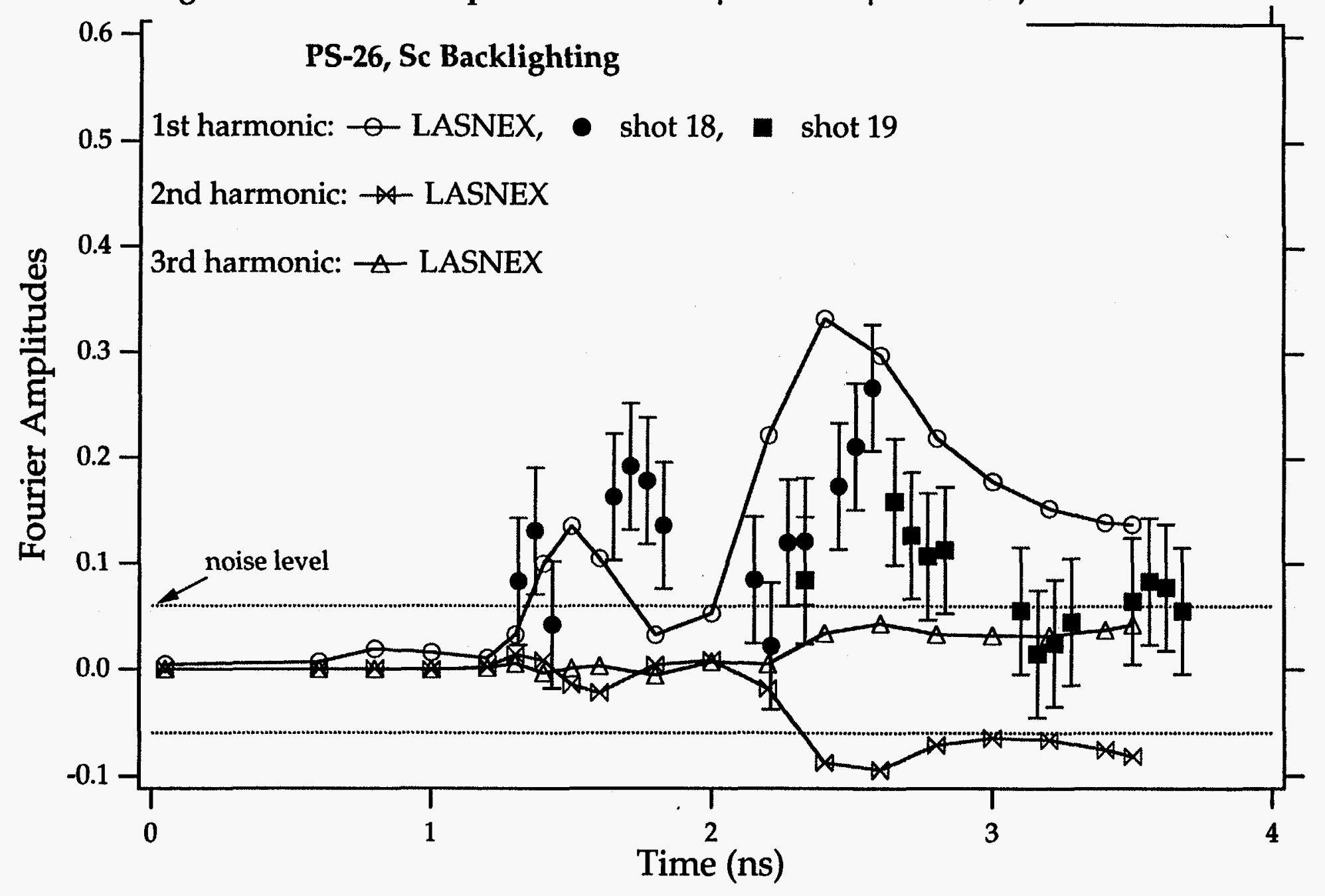


aluminum foil were both from Rayleigh-Taylor instability and on the cold surface. Linear coupling theory predicts that as a foil becomes much thinner than the perturbation wavelength, an instability on one surface will induce identical perturbation growth on all surfaces. The hot surface of the thin foils was ablatively stabilized, while the cold surface was not. The same instability may have acted as an ablative Rayleigh-Taylor instability on the hot side and as a classical RayleighTaylor instability on the cold side after the instability had strongly coupled across the interfaces.

The duel ablative/classical nature of the Rayleigh-Taylor instability in these experiments is highly speculative but has important implications if it is true. As the ablator on an ICF capsule becomes thinner toward the end of the pulse, modes on the ablation surface created by the Rayleigh-Taylor instability feed-into the interior, degrading the yield. The ablative stabilization prevents the growth of high-order modes on the ablation surface. One might assume then that only low order modes would feed-into the interior from the Rayleigh-Taylor instability. The data presented here suggests that if the shell is thin and the Rayleigh-Taylor instability couples strongly to the interior surface, high order modes could be found growing in the interior as well as low order. Again, this is only speculation but seems worth investigating in future shots.

Intermediate to the strongly and weakly coupled cases is a foil that moved from weak coupling to strong coupling during the experiment. This is the most interesting case because it most closely resembles the realistic ICF situation. 
Figure 5-9 shows the Fourier amplitudes for the $50 \mu \mathrm{m}$ foils shot with PS-35, which was experiment 15 . The intermediate coupling case is strongly dominated by the Rayleigh-Taylor instability and feed-in of the perturbation on the ablation surface back to the cold surface. The initial perturbation was placed on the cold surface, as in the other foils, but growth from the Richtmyer-Meshkov instability was very small compared to growth from the Rayleigh-Taylor instability after feed-out had occurred. One can clearly see Rayleigh-Taylor dominance in the data. There is an slight dip in the fundamental's Fourier amplitude until $3.5 \mathrm{~ns}$ from the Richtmyer-Meshkov growth and the feed-out moving toward the ablation surface. After $3.5 \mathrm{~ns}$, there is a large positive-phase growth of the fundamental and harmonics appear. This later growth is from the Rayleigh-Taylor instability. Acoustic waves are also present as they were in the thick packages, but are not clearly discernible in the data.

The thickness of the intermediate foil is optimal for Rayleigh-Taylor growth. The foil is not so thick that there is no feed-out, but not so thin that it quickly burns through without giving the Rayleigh-Taylor a chance to grow. The $50 \mu \mathrm{m}$ foil did not fall below the initial density of aluminum until around $7 \mathrm{~ns}$, well after the hohlraum radiation from the $4.5 \mathrm{~ns}$ pulse had turned off. One could argue that the larger growth is simply due to the longer pulse and not the thickness, the thick foil did not exhibit any feed-out seed on the ablation surface in side-on radiography even at 4.5 ns. If the thick foil had been driven by PS-35, there might have been some Rayleigh-Taylor growth, but probably not as much as 


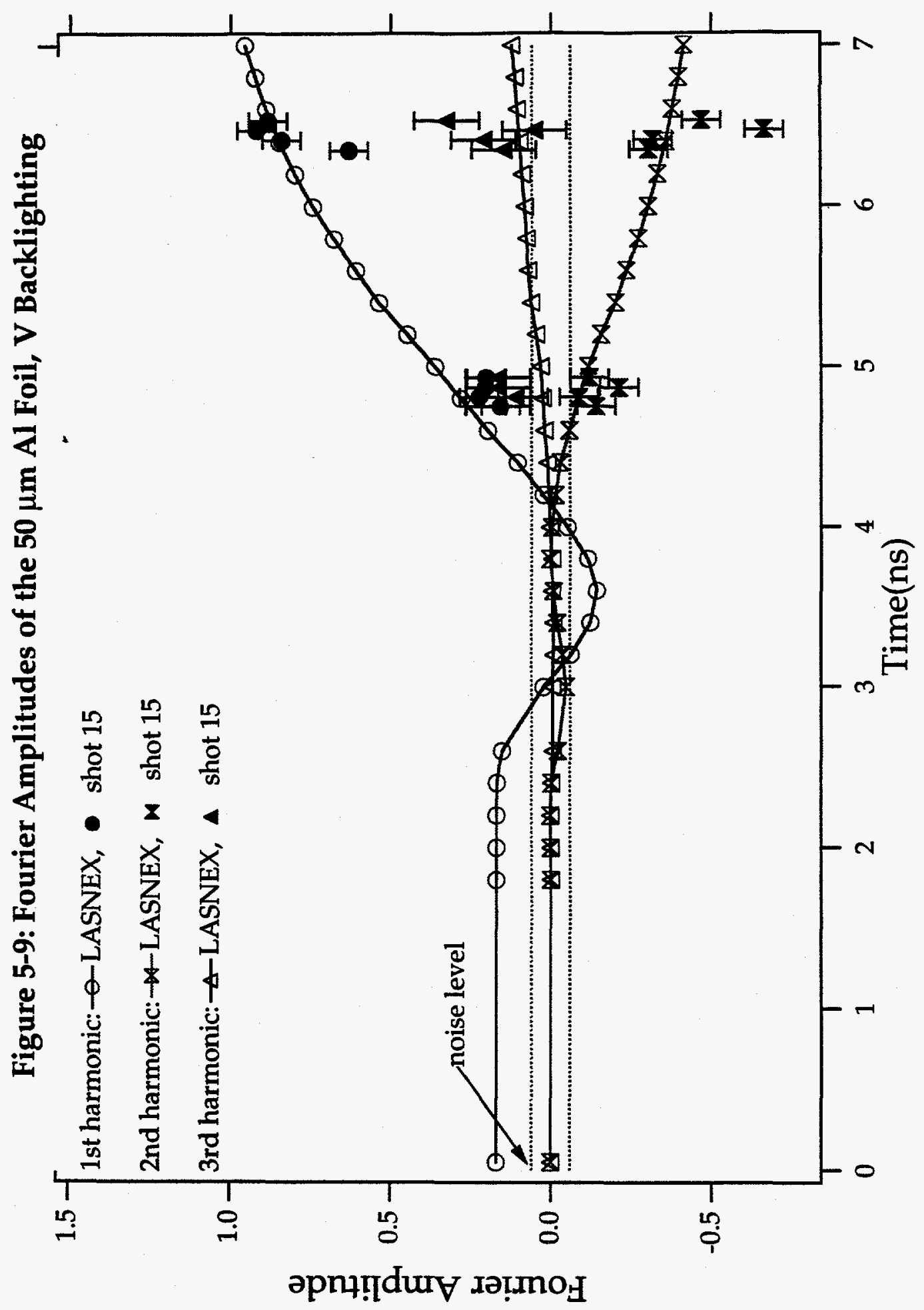


The $r$ and $z$ axes are to the same scale on all the plots and with respect to each other. Contours are not to the same scale. White lines are computational artifacts. Radiation is from the left. RT VP and RM VP refer to the Rayleigh-

Taylor and Richtmyer-Meshkov vortex pairs respectively. S symbolizes a shear vortex.

time $=2.7 \mathrm{~ns}$

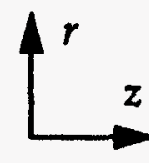

$z$ vorticity

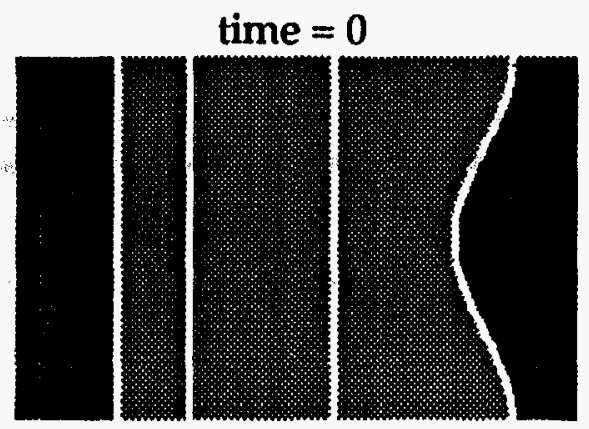

density time $=3.5 \mathrm{~ns}$
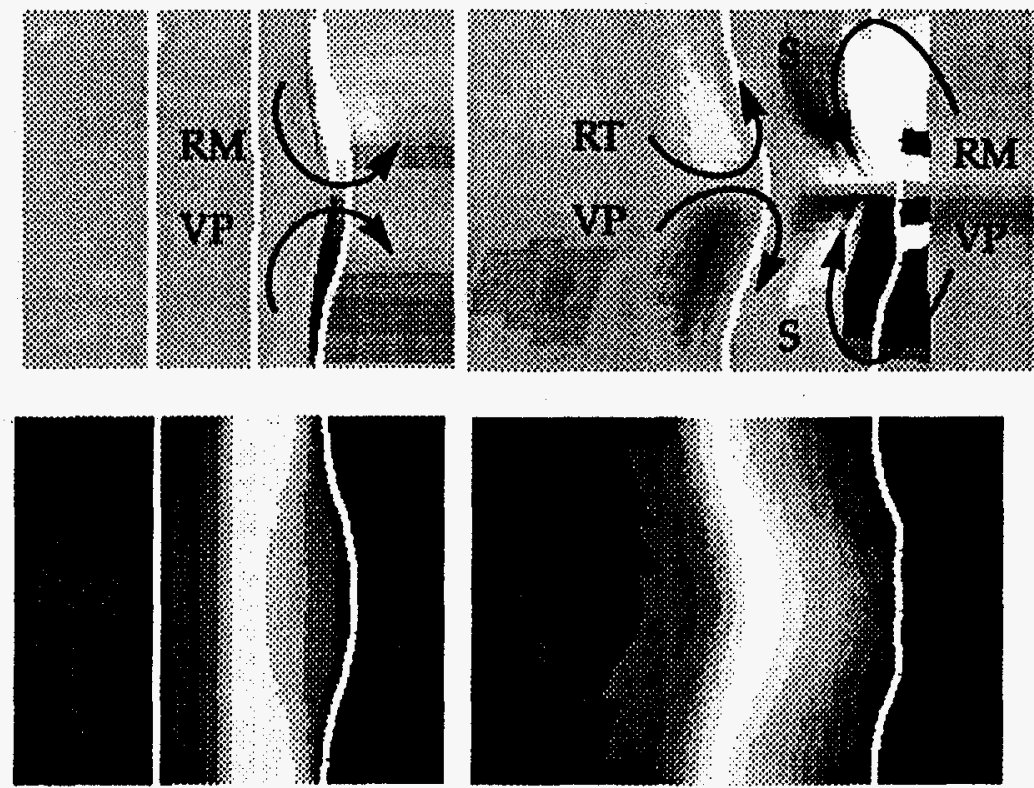
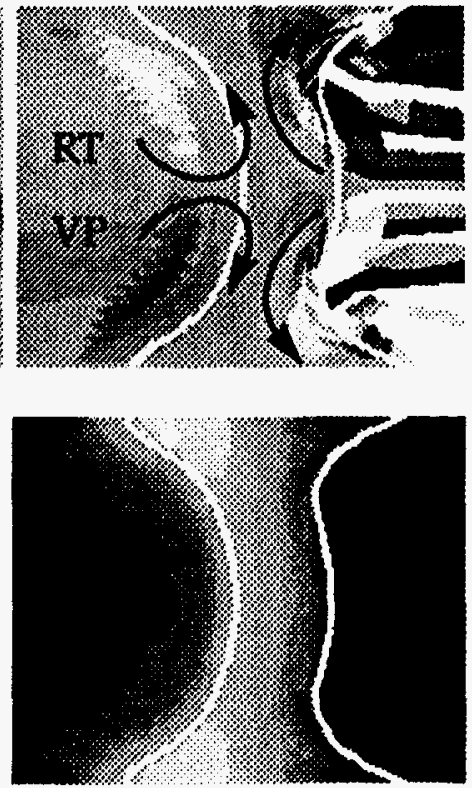
in the $50 \mu \mathrm{m}$ foil. The importance of thickness to feed-out and feed-in is demonstrated by the $50 \mu \mathrm{m}$ data.

Baltrusaitis' work revealed a very interesting fact about the RichtmyerMeshkov instability [Baltrusaitis]. If there were two instabilities with opposing vortex pairs, each one on an interface of a thin ribbon of fluid, one instability would always dominate. When the thickness of the fluid layer was small enough for the instabilities to strongly couple across the interfaces, the fluid could no longer support both instabilities. The vortex pair of the stronger of the two would remain but also be weakened. A natural question for the $50 \mu \mathrm{m}$ foil then, was how did the Rayleigh-Taylor and Richtmyer-Meshkov vortices interact as the foil narrowed, and which one dominated?

Figure 5-10 shows density and vorticity contour plots of the $50 \mu \mathrm{m}$ foil. Each density plot was made at the same time as the vorticity plot beneath it. The first plot on the left shows the initial package configuration, with the radiation source coming from the left.

By $2.67 \mathrm{~ns}$, the shock passed through the foil and a rarefaction wave may be seen moving back to the ablation surface. The perturbation carried by this wave is clearly evident in the density plot at this time. A Richtmyer-Meshkov instability took up residence on the cold surface, where its vortex pair may be seen in the vorticity plot. At $3.5 \mathrm{~ns}$, the perturbation reached the ablation surface and the entire foil was imprinted, creating a sinuous shape. The vortex pair of a Rayleigh-Taylor instability may be found at this time on the ablation surface in 
addition to the Richtmyer-Meshkov vortex pair on the cold surface. The vortex pairs of each instability rotated in the same direction, increasing the amplitude of the sinuous shape. The fluid velocity in the center of the foil from each instability moved in opposite directions and generated a shear vortex pair in-between the Richtmyer-Meshkov and Rayleigh-Taylor vortex pairs. Because the foil was still relatively thick, the shear was not excessive, and the two instabilities acted relatively independently.

Significant shear began to appear by $4.2 \mathrm{~ns}$, and by $5.4 \mathrm{~ns}$ the RayleighTaylor instability had coupled to the cold surface and overtaken the RichtmyerMeshkov instability. The vortex pair on the cold side then had a rotation complementary to the Rayleigh-Taylor vortex on the ablation surface. Both of these vortex pairs pulled fluid from the center of the figure to the edges, moving the package from a sinuous to a bubble and spike shape. The bubble is in the center of the figure, with spikes at the edges. The presence of the Richtmyer-Meshkov instability thus seemed to delay the transformation of the package into bubbles and spikes by moving fluid in an opposite direction to the Rayleigh-Taylor instability. These results are very similar to those of the Baltrusaitis paper. An interesting question is if instabilities on the two interfaces had complementary instead of opposing vortex pairs, would the nonlinear evolution of the foil be enhanced?

Evolution from the sinuous to bubble and spike shape was experimentally observed in the thin packages with side-on radiography, see Figure 5-3. From 1.8 to $1.98 \mathrm{~ns}$, the foil had an increasingly sinuous shape, with perturbations 
on each side. From 2.55 to $2.73 \mathrm{~ns}$, there was a distinct bubble and spike configuration. The spikes are the dark strips, the bubbles are the lighter spaces in between them. Although the Rayleigh-Taylor instability did not have the time to produce large growth in the thin foils, the evolution appears to be very similar to the 50 $\mu \mathrm{m}$ ' foil.

In addition to how feed-out varied with package thickness, the question of how a density jump affected it was both computationally and experimentally studied. Because the beryllium in the composite foils was a lower density than the aluminum and perturbation amplitude was the same in each, feed-out from differential acceleration was expected to be lower in the composite packages than in the pure aluminum packages. The density jump itself was not expected to directly affect the feed-out from differential acceleration, but was expected to lower the feed-out from interface coupling. The important question was then, how does a density jump affect the interface coupling?

To properly interpret the experimental data, one needed to ascertain whether differential acceleration or interface coupling was the dominant feed-out mechanism. This was accomplished by running calculations to estimate the individual amount of feed-out from each mechanism. The calculations involving differential acceleration are discussed first.

The thickness of each feed-out package was slightly larger if measured through the peak of the perturbation, and slightly smaller if measured through the trough. Two one-dimensional calculations were made of each of the four pack- 
ages with feed-out, one with the larger thickness and one with the smaller thickness. Both one-dimensional foils started at the same position. The location of the ablation fronts was defined at $\max \left|\frac{1}{\rho} \frac{\partial \rho}{\partial z}\right|$. Half the difference between the two ablation front positions was defined as the amplitude of the hot surface perturbation from differential acceleration. This manner of calculating feed-out from differential acceleration has a difficulty. In a two-dimensional foil, the RichtmyerMeshkov instability and acoustic waves move material from the peak of the perturbation to the troughs, saturating differential acceleration after about a nanosecond. These calculations thus provide an upper limit on the feed-out.

To calculate feed-out from interface coupling, a set of two-dimensional calculations were run using a pressure source to generate the shock instead of radiation. Acceleration effects were minimized in this fashion. Because the calculations were Lagrangian, perturbation amplitudes were easily obtained by subtracting the position of the top and bottom of each interface and dividing by two. Thinner foils were used in the pressure source calculations than in calculations with radiation. For the pure aluminum packages, $20 \mu \mathrm{m}$ was used in place of 35 $\mu \mathrm{m}$, and $35 \mu \mathrm{m}$ was used in place of $50 \mu \mathrm{m}$. For the foil with beryllium, $10 \mu \mathrm{m}$ of beryllium was used in both cases, and $12 \mu \mathrm{m}$ of aluminum replaced $32 \mu \mathrm{m}$. Interface coupling is strongly dependent on thickness. By the time the shock had reached the back surface of the packages, compression and ablation had significantly reduced foil thickness. Foil thicknesses at the time of shock arrival at the 
cold surface were estimated using the calculations with radiation. There are some problems with adjusting the thickness in an attempt to more accurately model the interface coupling. It is not entirely clear that matching thickness is more important than matching the total mass of the foil when simulating interface coupling with compressible fluids, but one cannot do both. Also the thickness of the radiatively driven foil is a function of time, which could not be simulated in the pressure source calculations.

The pressure sources were adjusted to create shocks with the same Mach number and density jumps as radiation. Calculations simulating the PS-35 shock worked well, but when the pressure was increased to simulate the PS-26 shock, numerical instabilities killed the calculation. The mesh was perfectly smooth with no perturbations of any kind, and it would still tangle around the shock before it reached the foil.

In order to avoid the mesh tangling problem, two calculations were used to simulate the PS-26 packages. The first was a two-dimensional run with a shock strength corresponding to PS-35. The second was a one-dimensional run with a shock strength corresponding to PS-26, which did not have the numerical instabilities. Perturbation growth on the "hot" surface was measured in the twodimensional case and multiplied by the ratio of postshock velocities of the one- to two-dimensional calculations. Use of the postshock velocity to estimate the growth from a stronger shock was based on the impulsive model and Mikaelian's theories. 
The final results are thus not exact in either case, but they provided a way of estimating each effect. They are plotted in Figure 5-11. In all cases, the differential acceleration is larger than the interface coupling, but the reader must keep in mind that differential acceleration will saturate faster than Figure 5-11 shows. Both mechanisms were probably important in the feed-out experiments, but this conclusion should not be generalized. Foils with other sizes and pulses could have differential acceleration or interface coupling as insignificant mechanisms.

With the understanding that changes in differential acceleration probably obscured the density jump effect on interface coupling, consider the peak amplitudes of the fundamental in Figures 5-4 and 5-7 and 5-8. The composite packages appear to have a smaller fundamental growth from the Rayleigh-Taylor instability, but why? The smaller growth could be in part from a smaller feed-out from the differential acceleration. It could also be in part because the acceleration of the composite package is slightly less than that of the pure aluminum. The two packages were initially designed to be mass matched, but due to an error in fabrication, the composite package was slightly thicker and more massive than the pure aluminum. The higher mass of the composite package resulted in a lower acceleration and slower Raleigh-Taylor growth than for the pure aluminum package, but it was not enough to account for the difference in peak amplitudes alone. A third factor is the perturbation on the aluminum/beryllium interface. The 

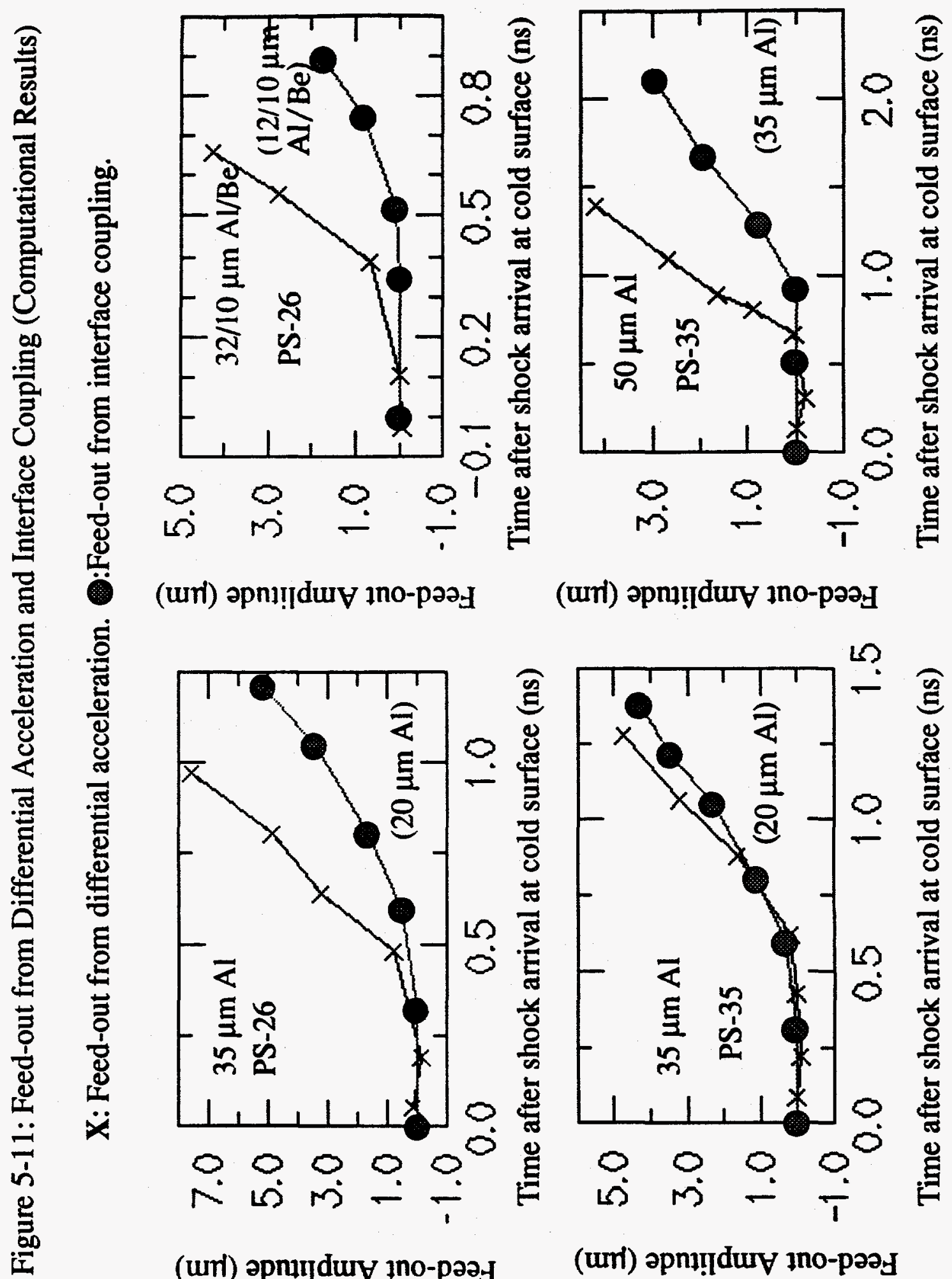

(uri) әрп!! dur ıno-рәә]

(ưi) әрn!! dur ıno-рәә]

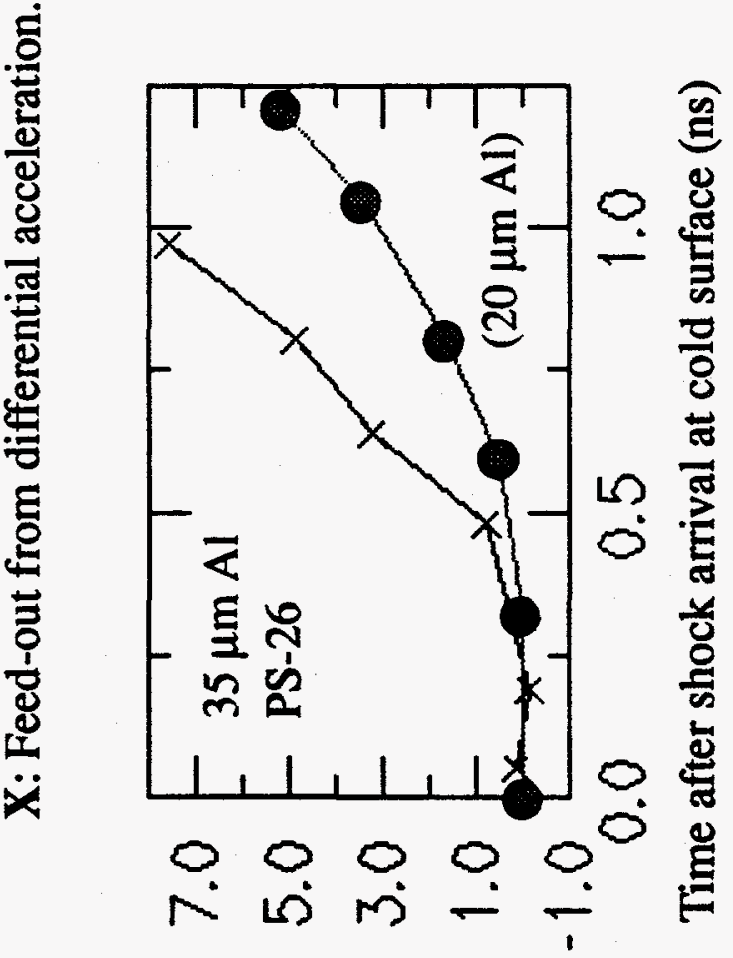

(uri) әрп!! dur ıno-рәә]

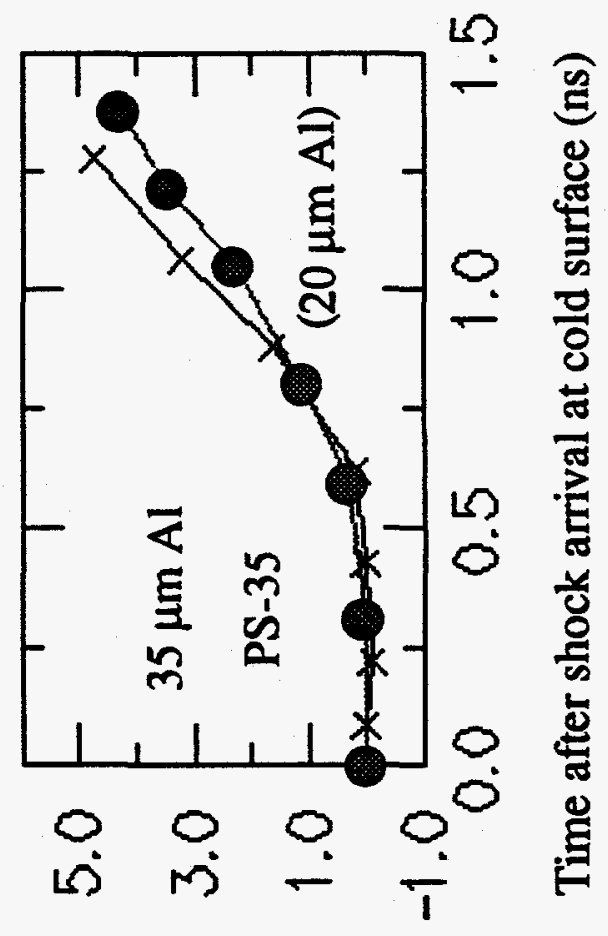

(uri) әpп!! durV ino-pәәу 
pressure source calculations described above implied that internal perturbations could be larger than the surface perturbations. Because the X-rays were interacting with perturbations on both surfaces, the amplitude of the fundamental was affected by this also. As a result, it is difficult to say exactly what effect the density jump had on the interface coupling feed-out in these experiments, other than it was not exceptionally large.

Because of the ambiguous nature of the data, additional calculations were made specifically to address the effect of a density jump on interface coupling. The calculations were two-dimensional pressure source calculations again. Two pressure source calculations were run with matched thicknesses, one with 35 $\mu \mathrm{m}$ of aluminum and the other with $25 \mu \mathrm{m}$ aluminum on the front and $10 \mu \mathrm{m}$ beryllium on the back. Again, a $4 \mu \mathrm{m}$ amplitude, $50 \mu \mathrm{m}$ wavelength perturbation was placed on the rear surfaces. A shock corresponding to the PS-35 drive pulse was sent through each.

Figure 5-12 shows the evolution of the five interfaces, including the aluminum/beryllium interface. The composite foil had slightly less feed-out than the pure aluminum, but not a lot. The density jump did not appear to affect the interface coupling significantly. Interestingly, the aluminum/beryllium interface had a larger perturbation than either the front or back surfaces until it reached saturation. The internal perturbation in the pure aluminum foil was likewise larger than the perturbations on either of its surfaces. The amplitude of the perturbation on the rarefaction wave could clearly be seen in each case and was almost the same. 

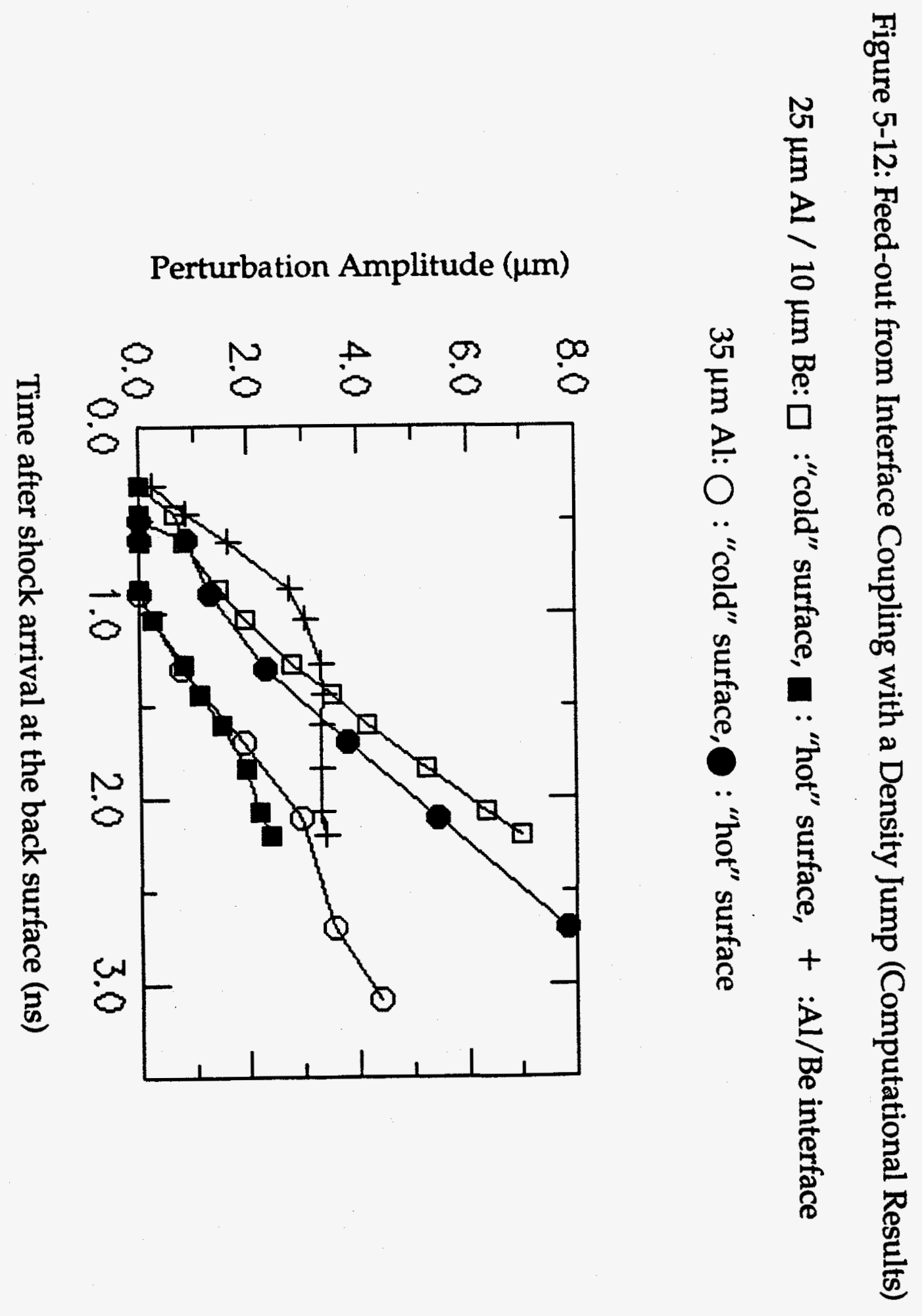
The implication of both the calculations and experiments was that a density jump did not have a large affect on the interface coupling feed-out.

Three final topics will now be discussed that may not relate to the feedout process. The first is the strong acoustic mode observed in many of the foils. Next, an experimental discrepancy, a large second harmonic is reviewed. Finally, the only major discrepancy between the experiments and calculations will be discussed.

An internal oscillatory mode was computationally observed in every foil and experimentally evident in the thick foils. The mode was of interest for two reasons, it might affect the seeding of the Rayleigh-Taylor and could represent a third instability in the foils. The oscillations in the thick foils had to be acoustic waves instead of an atmospheric type mode because the foils were not accelerating while the waves were present. Waves in the other feed-out packages occurred during times of acceleration and were some type of atmospheric type mode, either an acoustic, gravity, or Lamb mode. Both the Lamb and gravity modes offered interesting possibilities in that they could become unstable and grow, although the most likely possibility was always the acoustic mode which commonly accompanied the Richtmyer-Meshkov instability. Unfortunately, the modes were very difficult to observe in the calculations because the foils usually burned through before the waves completed one cycle. In addition, fluid motion from the Richtmyer-Meshkov and Rayleigh-Taylor instabilities complicates their study. 
To observe the waves more clearly, calculations were run with pure beryllium foils and PS-35. In beryllium, the wave could be observed over several periods while the package was still accelerating and could be seen continuing to oscillate well after the foils burned through.The foils were $50 \mu \mathrm{m}$ thick with perturbation amplitudes of $0.5 \mu \mathrm{m}$. To increase the foil lifetime during the pulse and decrease shock strength to assure linearity, total laser energy was decreased to 15 $\mathrm{kJ}$. Wavelengths from 5 to $50 \mu \mathrm{m}$ were run. There was no guarantee that the waves in the beryllium calculations were the same as those of the feed-out foils, but the feed-out waves are unobservable over multiple wavelengths.

The term "turn around" will be used here to designate points where the first derivative of the fundamental Fourier amplitude changed sign, such as at 3.5 ns in Figure 5-2. The results seemed to confirm that these modes were stable, acoustic modes. The amplitude of the waves oscillated randomly with time around the amplitude at the first turn around and was a function of the wavelength, although this cannot be explained. There was no obvious growth of the amplitude over time, so the waves did not appear to be internal instabilities, such as a Lamb mode. The time between shock arrival at the cold surface and the first turn around was not indicative of a half period. A half period was more accurately estimated as the time between the first and second turn around.

The periods approximately matched those for an acoustic mode, but, after the calculations were completed, a possible error was discovered. Linear theory suggested that the physics of the beryllium packages may not have supported 
the existence of gravity modes, unlike the feed-out foils.

An experimental discrepancy occurred in the $35 \mu \mathrm{m}$ foils shot with PS26 and there was not a sufficient number of shots to explore it thoroughly. A large second harmonic appeared in two of the three shots taken with this foil, which disagreed with previous shot data and computational predictions. Figure 5-13 shows the data for shots 8 and 17, which were identical to shot 3 in Figure 5-4, with the exception that a scandium backlighter was used at $4.3 \mathrm{keV}$ instead of titanium at $4.7 \mathrm{keV}$. The difference in backlighter energies between shot 3 and shots 8 and 16 accounts for the slightly lower initial Fourier amplitudes of the packages, as the opacities were a little different. Figure 5-14 shows raw data from the framing camera for shots 3 and 8 . The frequency doubling is clearly seen in one shot but not the other.

Two particularly unusual aspects of this large second harmonic were that it attained an amplitude much larger than the fundamental, and this large amplitude was early in time, just after the shock hit the cold surface. These two facts suggest this was not a non-linear mode coupling effect, such as described by Haan's theories. The second harmonic decreased rapidly in amplitude after about $1.8 \mathrm{~ns}$, suggesting that the effect was short lived. Several possibilities were considered.

A machining error could have occurred in package fabrication, resulting in an initial second harmonic. A LASNEX calculation was run which included an initial second harmonic with the fundamental. The second harmonic's ampli- 


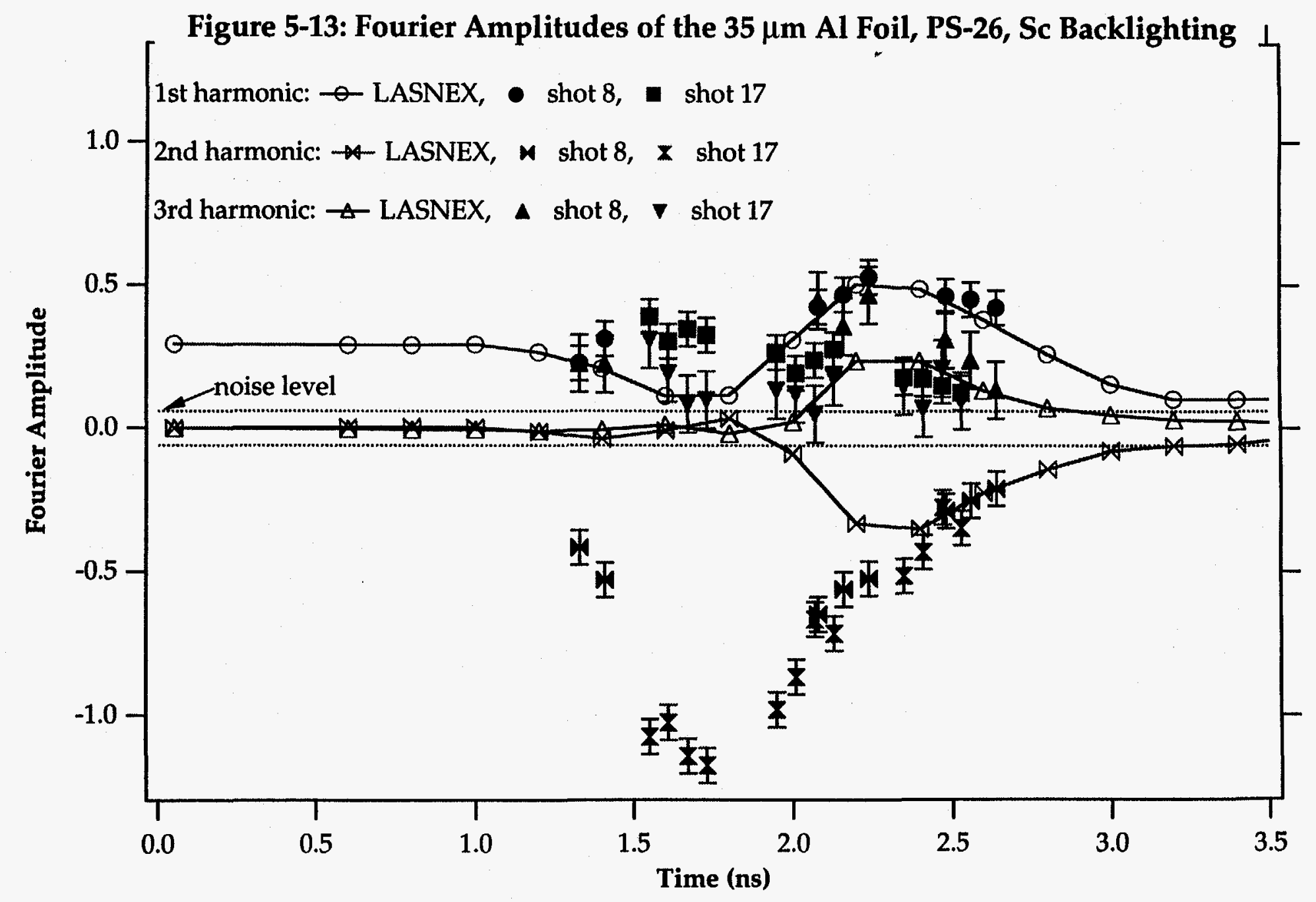



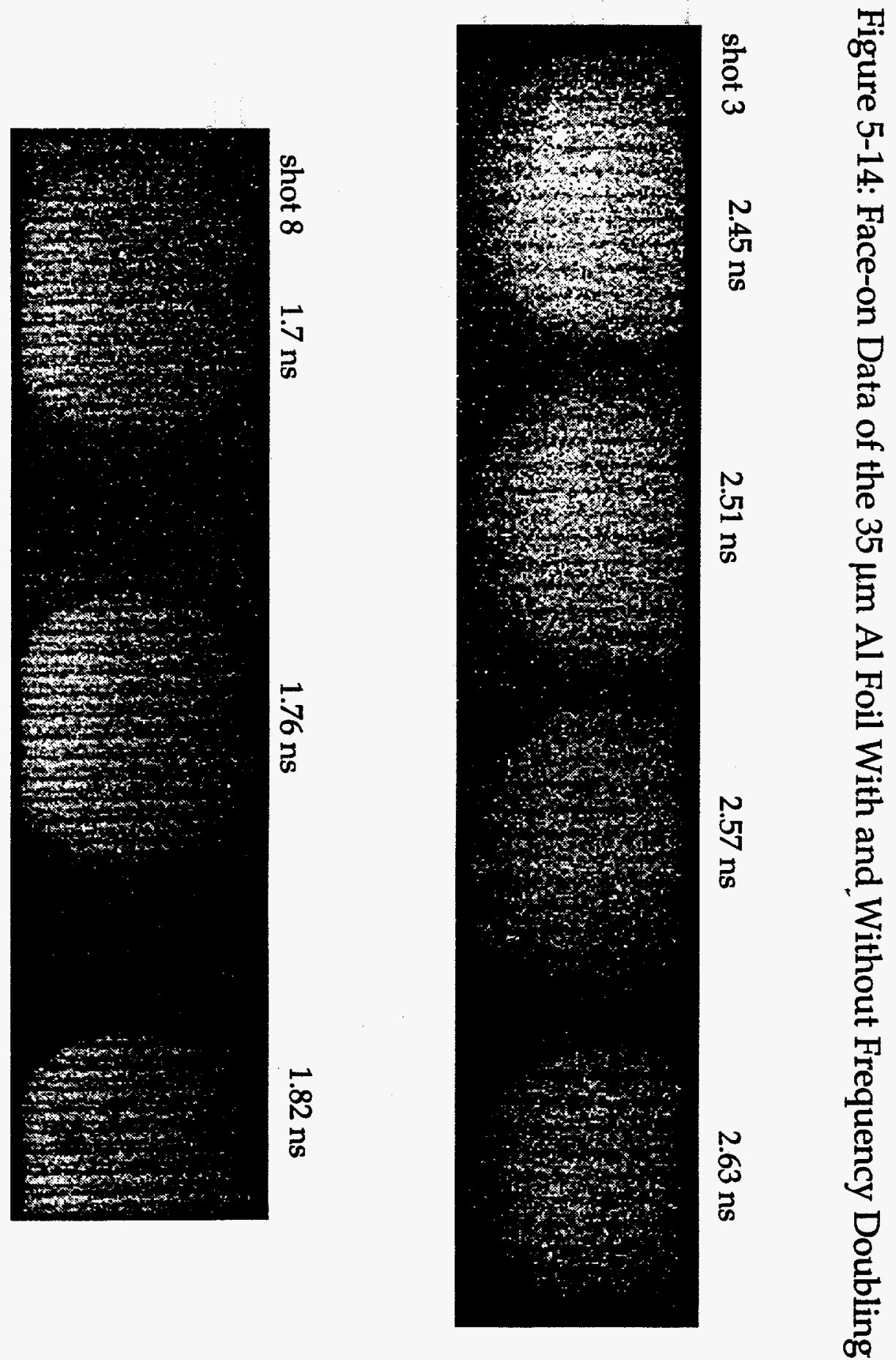
tude was set to ten percent of the amplitude of the fundamental. Subsequent Fourier analysis showed no growth of the second, and even a slight decrease until about $1.8 \mathrm{~ns}$. The final result was very similar to Figure 5-4. In addition, before the targets were fielded, the perturbations on some of the packages were scanned and recorded. Preliminary Fourier analysis of the available scans did not indicate an initial second harmonic present.

Excessive preheat from the gold M-band was considered a possibility because it would fit the early time nature of the phenomenon. The perturbation troughs would be preheated slightly more than the peaks. The preheat differential could result in a discharge of vaporized material by the troughs, which would form into jets of material. The jets and perturbation peaks could form a large second harmonic.

Another LASNEX calculation was run with the intensity of all radiation bins from $2.2-8.4 \mathrm{keV}$ increased an by order of magnitude. Fourier analysis showed a time history similar to Figure 5-4, with the difference that the increase and decrease in the harmonic amplitudes was much steeper around 2.4 ns. Preheat did not appear to be the answer.

A third possibility was entertained. Schappert and Hollowell found a large second harmonic in their experiments which was attributed to a high order mode coupling into the second [Hollowell]. However, the second harmonic in this case appeared late in time. RAGE was more capable of modeling this effect than LASNEX, and Hollowell ran a RAGE calculation simulating the $35 \mu \mathrm{m}$ aluminum 
package. The perturbation was modeled by square zones approximately $0.6 \mu \mathrm{m}$ in thickness in a stair step fashion, creating a high order mode similar to the machining process. No large second harmonic appeared, either as observed in the Schappert or feed-out shots. The source of the large second harmonic remains unknown.

A discrepancy was also found between the calculations and experiments in the case of the $35 \mu \mathrm{m}$ foil shot with PS-35, see Figure 15. LASNEX predicted much larger modal amplitudes than observed during late times, after the package had burned through at $5 \mathrm{~ns}$. The reason for this is unclear. It was suggested that this discrepancy could be accounted for by tilting of the spikes out of the direct line of sight of the camera. Side-on radiographs of the other feed-out packages do not show such a tilt in the spikes, although bowing is evident. Although the foil shows a large amount of bending as it lifts off from the hohlraum, the spikes appear to all be perpendicular to the hohlraum and the drive, both in the thin and thick foil cases. Hollowell and Schappert had a similar discrepancy between RAGE calculations and experiments [Schappert, Hollowell]. 


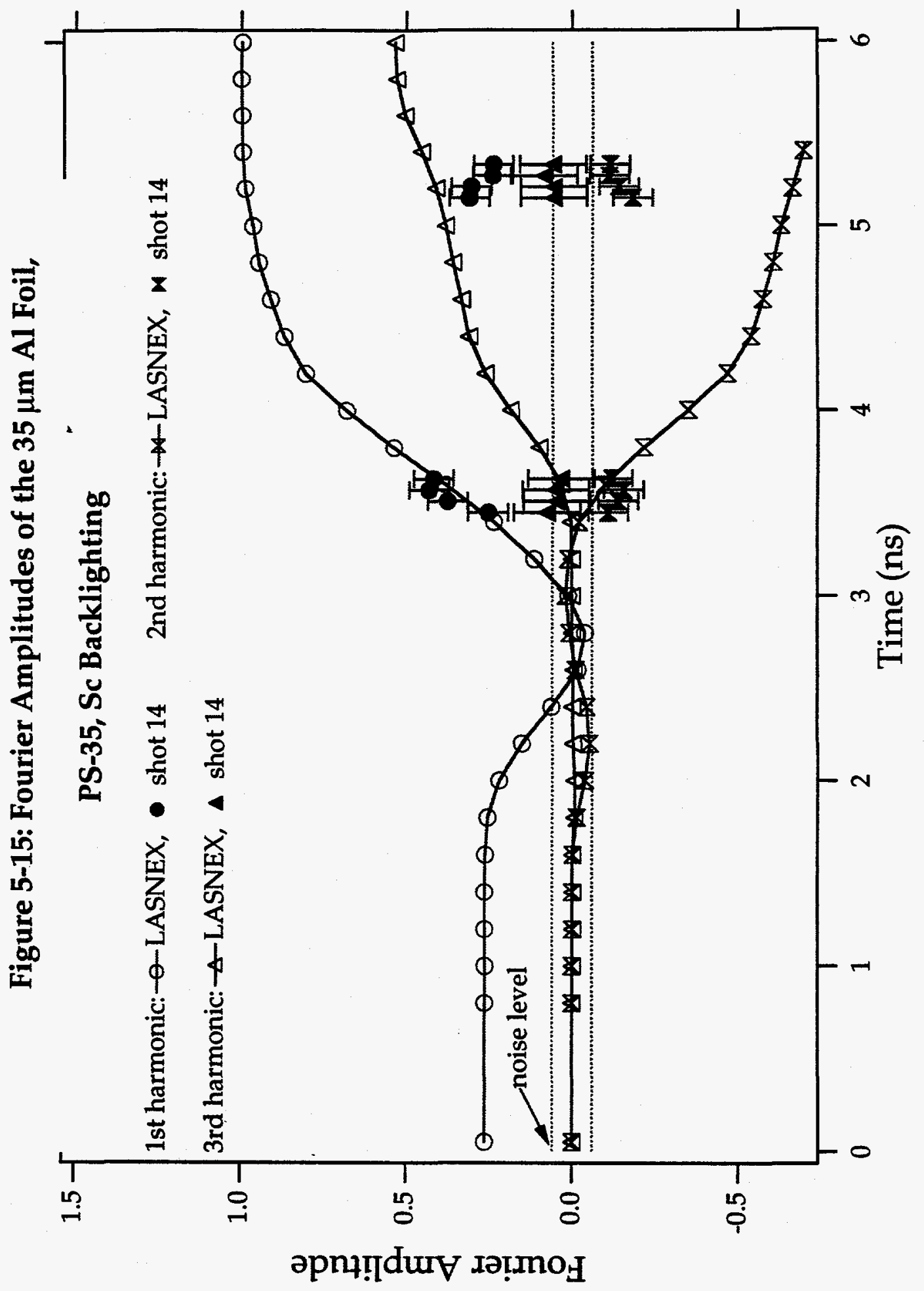




\section{Conclusion}

Feed-out is the communication of a perturbation from the cold surface of a radiatively driven foil to the ablation surface, and its subsequent growth. Differences in feed-out are believed to be partially responsible for the superior robustness of beryllium NIF capsules over plastic capsule designs in regard to interior perturbations. Feed-out is an important hydrodynamic effect in ICF because it couples the large internal perturbations with the long-duration, strong ablation surface instability.

In order to better understand the nature of feed-out, an experimental and computational campaign was undertaken to study the effects of a density jump and variations in coupling on the phenomena. Planar aluminum foils with a perturbation on the cold surface were driven with radiation from a hohlraum. Thickness and pulse length were adjusted to observe the following cases: weakly coupled hot and cold interfaces, strongly coupled interfaces, and an intermediate case which moved from independent Rayleigh-Taylor and Richtmyer-Meshkov instabilities to coupled instabilities as the package burned through. Composite packages of aluminum ablators and beryllium payloads were used to observe the effect of a density jump. The beryllium was transparent to the backlighter, so feedout into the aluminum was visible. This was the first campaign known to the author undertaken specifically to investigate feed-out in a regime relevant to ICF.

The existence of the feed-out phenomena was confirmed experimentally for the first time in radiatively driven foils using side-on and face-on radiog- 
raphy. The thickness and pulse length were found to be crucially important, as they determined the level of interface coupling. The thick foil showed weak coupling of the cold and hot interfaces, while the thin foil showed a strong and fast coupling. Because of the short pulse and package thickness, only the RichtmyerMeshkov instability was present in the thick foil. Both Richtmyer-Meshkov and Rayleigh-Taylor instabilities were present in the thin foil. The cold surface perturbation was observed feeding-out into the aluminum ablator from the beryllium payload in the thick aluminum/beryllium composite package. In the thin composite case, a curious peak in the fundamental amplitude was observed during the time feed-out was expected. The peak was the opposite phase from what one would expect of feed-out. There was some evidence that the Rayleigh-Taylor instability in the thin foil acted as an ablative instability on the ablation surface and as a classical instability on the cold surface. If the Rayleigh-Taylor actually had a duel behavior, then the instability could generate higher harmonics in the interior of ICF capsules than on the exterior ablation surface.

The package with intermediate coupling showed a large amount of Rayleigh-Taylor growth. The physics of this package was dominated by the Rayleigh-Taylor growth feeding-back to the cold surface. Calculations showed the development of two relatively independent instabilities in the beginning, a Richtmyer-Meshkov on the cold surface and Rayleigh-Taylor on the ablation surface. During this time, the foil maintained a sinuous shape. As the package burned through and the perturbations grew, the vortex pair of the Rayleigh-Taylor 
became dominant over the Richtmyer-Meshkov vortex pair, evolving the foil into a non-linear bubble and spike shape.

Calculations indicated that both differential acceleration and interface coupling were important to the feed-out process in the experiments presented here. Interface coupling feed-out is the seeding of the Rayleigh-Taylor instability by the Richtmyer-Meshkov instability coupling to the ablation surface. Differential acceleration feed-out is the seeding of the Rayleigh-Taylor instability by differences in the acceleration of the fluid columns running through the troughs and peaks of the cold surface perturbation. Experimental data and calculations indicated that, contrary to the original hypothesis, a density jump may not greatly affect feed-out from interface coupling. There is the possibility of a moderate to small effect. The density jump was not expected to affect feed-out from differential acceleration, however, the composite packages had a smaller mass differential between the peaks and troughs of the perturbation because beryllium is not as dense as aluminum. The lower mass differential should have resulted in less feedout from differential acceleration.

Three interesting observations were made that do not necessarily relate to feed-out. Large oscillatory modes were computationally observed in all the foils and experimentally observed in the thick foils. These modes were believed to be an acoustic wave in the thick foils and an acoustic-type atmospheric mode in the other packages. Secondly, a large second harmonic was observed in two of the three thin foil shots with the 2.2 ns pulse. This large harmonic did not agree with 
the first experimental data or the calculations. Lastly, there was a discrepancy between the computationally predicted growth during late times of the $35 \mu \mathrm{m}$ foil shot with the $4.5 \mathrm{~ns}$ pulse and the data. The data showed much less growth than predicted. The reasons for the large second harmonic and low growth in the PS-35 foil are unknown. Possible reasons are discussed in chapter 5. A summary of the experiments and their results is shown in Table 5-1 below.

Table 5-1:

\begin{tabular}{|l|l|}
\hline $\begin{array}{c}\text { Package } \\
\text { Thickness } \\
\text { Leng), Pulse } \\
\text { Length (ns) }\end{array}$ & \multicolumn{1}{|c|}{ Results } \\
\hline $86 \mathrm{Al}, 2.2$ & $\begin{array}{l}\text { Weak interface coupling. Richtmyer-Meshkov instability } \\
\text { only. Strong acoustic waves. Only the fundamental mode } \\
\text { was present. This package showed evolution of the feed-out } \\
\text { seed. }\end{array}$ \\
\hline $35 \mathrm{Al}, 2.2$ & $\begin{array}{l}\text { Strong, fast interface coupling. Strong second harmonic } \\
\text { observed in two of the three shots. Feed-out of the perturba- } \\
\text { tion to the ablation front experimentally confirmed with } \\
\text { side-on radiography. }\end{array}$ \\
\hline $84 \mathrm{Al} / 10 \mathrm{Be}$, & $\begin{array}{l}\text { Observed feed-out of the perturbation from beryllium into } \\
\text { aluminum. Only the fundamental mode was present. } \\
\text { Strong acoustic waves. }\end{array}$ \\
\hline $32 \mathrm{Al} / 10 \mathrm{Be}$, & $\begin{array}{l}\text { Early time peak observed in fundamental, possibly feed-out } \\
\text { of perturbation into the aluminum, but wrong phase. Data } \\
\text { implied that harmonics were only in the beryllium and that } \\
\text { the Rayleigh-Taylor acted ablatively on the hot surface and } \\
\text { classically on the cold surface after feeding-back into the } \\
\text { foil. }\end{array}$ \\
\hline $502 \mathrm{Al}, 4.5$ & $\begin{array}{l}\text { Package moved from weak to strong interface coupling. } \\
\text { Rayleigh-Taylor vortex pairs dominated package evolution, } \\
\text { displacing the Richtmyer-Meshkov vortex pairs. }\end{array}$ \\
\hline $35 \mathrm{Al}, 4.5$ & $\begin{array}{l}\text { Data showed much lower amplitude perturbations than } \\
\text { computationally predicted for times after the pulse was off. }\end{array}$ \\
\hline
\end{tabular}


The results of this work suggest that greater thickness during the implosion may be part of the reason why beryllium capsules are superior to plastic. The larger density jump between the fuel and ablator in beryllium probably did not have as much of an effect as the thickness in reducing the feed-out. Most importantly, feed-out has been computationally and experimentally demonstrated to couple perturbations on the cold surface of a radiatively driven foil with the Rayleigh-Taylor instability on the ablation surface. From this, one can assume that it will also couple the large DT ice perturbations in ICF capsules to the Rayleigh-Taylor instability on the ablation surface. Such coupling will require either further reduction in the ice perturbation amplitudes or design of feed-out resistant capsules, such as the beryllium capsule.

Some comments will now be made in regard to what work remains to be done. The highest priority needs to be feed-out calculations in the NIF parameter space. Preliminary work suggests that feed-out on NIF may be quite different from NOVA.

Better experiments could be performed in order to quantitatively determine how thickness affects feed-out. The packages proposed would be composed of an aluminum ablator and beryllium payload, with a perturbation on the cold, beryllium surface. Using face-on radiography, one could look at packages with different thicknesses of beryllium, but the same total thickness. The pulse should be short so that only the Richtmyer-Meshkov instability would be induced. The amplitude of the feed-out seed could be observed as it moved toward the ablation 
surface at different positions in the package. The reduction in perturbation amplitude with position could be recorded.

Perhaps one could determine if the Rayleigh-Taylor instability was generating harmonics on the cold surface using thin packages with a beryllium ablator and aluminum payload. This would confirm that the Rayleigh-Taylor instability was acting as an ablative instability on the hot surface and as a classical instability on the cold surface. A perturbation could be placed on the hot or cold surface, but preferably the cold surface so data could be compared to the data presented here.

Although probably not of interest to the ICF community, experiments exploring different aspects of interface coupling would be of scientific interest. Freeze-out from Richtmyer-Meshkov and Rayleigh-Taylor interface coupling has had very little experimental investigation. Mikaelian proposed some experiments along these lines [Mikaelian 1996]. Investigations involving Richtmyer-Meshkov interface coupling with a reflected shock instead of a reflected rarefaction wave could be interesting. Perturbations on a shock decay, whereas perturbations on a rarefaction wave do not, so the interface coupling might be weaker when the reflected wave was a shock. In all the experiments in this dissertation, the reflected wave was a rarefaction. There is also the case of total shock transmission at the interface described by Yang. This case might not produce any interface coupling. 


\section{Appendix: Details of Individual Shots}

Following in Tables 1-7 is a summary of the feed-out experiments performed on NOVA. There was a great deal of information collected on each shot, such as the actual pulse shape generated by the laser and individual beam line energies, but only the more important details are included in the tables below.

Tables 1 and 2 provide information on the packages and the main pulse. The shots are listed in the order in which they were actually fielded, and so the shotnames do not necessarily proceed in order. Each shot had three designation numbers, a shotnumber corresponding to the number of shots the laser had performed up to that point, a target identification number, to designate which package was being shot, and a shotname. For simplicity, only the shotname is provided. The $2.2 \mathrm{~ns}$ pulse listed is PS-26, while the $4.5 \mathrm{~ns}$ is PS-35. Both of these were described in the introduction. The percent spread in beam energy represents the root mean squared deviation of the individual beam energies from the average. On shot 15, one of the beams had only about a third of the expected energy while another almost did not fire.

Table 3 provides information on the primary diagnostic in SIM 4. The optimal gain for the camera was found by trial and error. The timing pulse delay is the delay time added to the circuit between the NOVA diagnostic trigger and the camera, so the camera calls time zero when the laser starts to fire. This timing was always somewhat in question. The strip timings are delays placed on individual strips after the beginning of the experiment. 
Table 4 details problems experienced with each shot and the many lessons learned. The references in the table are to SIM 4 data, unless otherwise specified.

During shots 1 and 2, the first two strips from the X-ray camera were completely saturated. The problem was corrected by placing radiation shields on all the following targets. The addition of a collimator with $50 \mu \mathrm{m}$ holes also helped reduce noise. In shot 3, a piece of target debris punctured through several filters in the nose cone of the GXI, and almost damaged the microchannel plate. The beryllium filter on SIM 4 was then doubled in thickness to protect the diagnostic.

Another concern was adjustment of the gain on the FXI or GXI. If the gain was too low, the image would not be visible; too high, and the film would be saturated. Trial and error were used to determine the best setting for a given package. On shots 4 and 5, the FXI gain was too low and some data was lost. Low gain was also a problem on shot 20 . The FXI was found to be a superior camera to the GXI's and was used exclusively toward the end of the experimental campaign. It did not have as many strips misfire as the GXI's.

Certain backlighters were found to be more desirable than others. Generally, a slightly higher energy backlighter than necessary was initially used to insure that transmission was high enough. Backlighter energy was then slowly decreased to increase the intensity ratio. For the thin packages, Ti was used first, then Sc. For the thick packages, Fe was changed to $\mathrm{Mn}$. The difference in back- 
lighters was most noticeable in the side-on shots $11,12,9$ and 10. Copper was found to be much too dim compared to Fe.

Package thickness became a concern after the $6 / 96$ series of shots. A measure of package thickness was attempted in metrology, and the thicknesses were found to be slightly different than expected. Target fabrication measured the thicknesses on the $8 / 96$ series side-on, but not face-on packages. Shots 11 and 9 were suppose to be with $35 \mu \mathrm{m}$ thick packages, but they were not, because of fabrication error. Target fabrication suspected there was some kind of systematic error in the process resulting in an additional 4-5 $\mu \mathrm{m}$ thickness for all the packages, at least for the $8 / 96$ shots. The lathe used to machine the packages was much more accurate than the 4-5 $\mu \mathrm{m}$ error. This error could have entered the machining process when the copper substrate was removed from the lathe, placed in the vacuum chamber, then placed back on the lathe. As this systematic error is a suspicion and cannot be confirmed, the requested thicknesses are listed in the tables instead of the suspected thicknesses, with the exception of shots 11 and 9 , which were measured.

Streak camera data was lost in shot 20 due to a lead shield falling on the crystal and interfering with X-ray transmission to the streak camera. The shield had previously been Scotch taped by the student to a baffle in the spectrometer in order to reduce noise, but an insufficient amount of tape was used. In future shots the shielding was removed.

In shot 21, the diagnostic alignment was off. For shot 22 , we removed 
the cap on the FXI, exposing the collimator and pinholes. Alignment of the diagnostic was much easier using the holes in the collimator instead of the featureless face of the cap, but it also ran the risk of damaging the collimator or pinholes more easily. The alignment on shot 22 was good, with no damage to the camera.

Table 5 shows information on the SIM 3 and 6 diagnostics. The terms "TP" and "Cam" delays refer to the timing pulse and camera delays. The camera delay is how long the diagnostic waits from the beginning of the experiment to begin taking pictures. The timing pulse delay is the same as in Table 3. Both diagnostics ran over the same time frame as the backlighter. The GXI strips could be set to the same delays as SIM 4, but they did not have to be. The main purpose of these two diagnostics was to deduce if the backlighter was at fault if there was poor data from SIM 4.

Table 6 contains information on the backlighter element, energy on the backlighter and the energy of the $\mathrm{He}-\alpha$ line. Two flat-top pulses were used to drive the backlighter. One was a 3 ns pulse, referred to as (64)*PS1-03, while the 2 ns pulse was (26)*PS1-02. The 2 ns pulse provided more intensity as the laser power was higher. The backlighter beams were turned on 200 ps before the first strip on the X-ray camera fired, providing enough time to generate a plasma on the backlighter disk before pictures were taken.

Data on diagnostic alignment and laser pointing are provided in Table 7. All three diagnostics were pointed at the backlighter. Although SIM 4 was the package diagnostic, it looked at the backlighter through the package. Experience 
showed that aligning SIM $3444 \mu \mathrm{m}$ above the actual position of the backlighter was more accurate than requesting the exact number. Why this worked is unknown. 
Table 1: Packages

\begin{tabular}{|c|c|c|c|}
\hline Shotname & Date & Thickness (mm) & Face or Side-on \\
\hline 1 & $4 / 26 / 96$ & $86 \mathrm{Al}$ & face \\
\hline 2 & $4 / 26 / 96$ & $84 \mathrm{Al} / 10 \mathrm{Be}$ & face \\
\hline 3 & $4 / 26 / 96$ & $35 \mathrm{Al}$ & face \\
\hline 4 & $4 / 26 / 96$ & $32 \mathrm{Al} / 10 \mathrm{Be}$ & face \\
\hline 5 & $4 / 26 / 96$ & $32 \mathrm{Al} / 10 \mathrm{Be}$ & face \\
\hline 6 & $6 / 18 / 96$ & $86 \mathrm{Al}$ & face \\
\hline 7 & 6/18/96 & $86 \mathrm{Al}$ & face \\
\hline 8 & $6 / 18 / 96$ & $35 \mathrm{Al}$ & face \\
\hline 15 & $8 / 96$ & $50 \mathrm{Al}$ & face \\
\hline 14 & $8 / 96$ & $35 \mathrm{Al}$ & face \\
\hline 13 & $8 / 96$ & $35 \mathrm{Al}$ & face \\
\hline 11 & $8 / 96$ & $40 \mathrm{Al}$ & side \\
\hline 12 & $8 / 96$ & $85 \mathrm{Al}$ & side \\
\hline 9 & $8 / 96$ & $41 \mathrm{Al}$ & side \\
\hline 10 & $8 / 96$ & $86 \mathrm{Al}$ & side \\
\hline 16 & $3 / 97$ & $35 \mathrm{Al}$ & face \\
\hline 17 & $3 / 97$ & $35 \mathrm{Al}$ & face \\
\hline 18 & $3 / 97$ & $32 \mathrm{Al} / 10 \mathrm{Be}$ & face \\
\hline 19 & $3 / 97$ & $32 \mathrm{Al} / 10 \mathrm{Be}$ & face \\
\hline 20 & $3 / 97$ & $84 \mathrm{Al} / 10 \mathrm{Be}$ & face \\
\hline 21 & $3 / 97$ & $84 \mathrm{Al} / 10 \mathrm{Be}$ & side \\
\hline 22 & $3 / 97$ & $32 \mathrm{Al} / 10 \mathrm{Be}$ & side \\
\hline
\end{tabular}


Table 2: Main Pulse

\begin{tabular}{|c|c|c|c|c|}
\hline Shotname & $\begin{array}{l}\text { Main Pulse } \\
\text { Length (ns) }\end{array}$ & $\begin{array}{c}\text { Energy in } \\
\text { Hohlraum (kJ) }\end{array}$ & $\begin{array}{l}\text { Requested } \\
\text { Energy (kJ) }\end{array}$ & $\begin{array}{l}\text { \% Spread in } \\
\text { Beam Energy }\end{array}$ \\
\hline 1 & 2.2 & 24.4 & 25.6 & 8.33 \\
\hline 2 & 2.2 & 25.0 & 25.6 & 3.72 \\
\hline 3 & 2.2 & 27.5 & 25.6 & 3.54 \\
\hline 4 & 2.2 & 24.8 & 25.6 & 3.32 \\
\hline 5 & 2.2 & 25.3 & 25.6 & 2.50 \\
\hline 6 & 2.2 & 25.1 & 24.0 & 2.49 \\
\hline 7 & 2.2 & 22.6 & 24.0 & 3.03 \\
\hline 8 & 2.2 & 25.3 & 24.0 & 2.93 \\
\hline 15 & 4.5 & 18.3 & 24.0 & 45.77 \\
\hline 14 & 4.5 & 21.4 & 24.0 & 9.69 \\
\hline 13 & 2.2 & 28.5 & 24.0 & 7.00 \\
\hline 11 & 2.2 & 24.1 & 24.0 & 7.13 \\
\hline 12 & 2.2 & 25.2 & 24.0 & 8.62 \\
\hline 9 & 2.2 & 24.2 & 24.0 & 7.45 \\
\hline 10 & 2.2 & 23.1 & 24.0 & 8.23 \\
\hline 16 & 2.2 & & 24.0 & \\
\hline 17 & 2.2 & 24.2 & 24.0 & 12.08 \\
\hline 18 & 2.2 & 25.3 & 24.0 & 2.52 \\
\hline 19 & 2.2 & 23.1 & 24.0 & 1.70 \\
\hline 20 & 2.2 & & 24.0 & \\
\hline 21 & 2.2 & 25.4 & 24.0 & 2.26 \\
\hline 22 & 2.2 & 24.0 & 24.0 & 3.27 \\
\hline
\end{tabular}


Table 3: SIM 4 Diagnostic

\begin{tabular}{|c|c|c|c|c|}
\hline $\begin{array}{l}\text { Shot- } \\
\text { name }\end{array}$ & $\begin{array}{l}\text { SIM 4 Camera } \\
\text { \& Gain (V) }\end{array}$ & $\begin{array}{c}\text { SIM 4 } \\
\text { Filters (mil) }\end{array}$ & $\begin{array}{l}\text { SIM } 4 \text { Timing } \\
\text { Pulse Delay (ns) }\end{array}$ & $\begin{array}{l}\text { SIM } 4 \text { Strip Timings } \\
\text { (ns) }\end{array}$ \\
\hline 1 & GXI $3 /-300$ & $11 \mathrm{Be} 0.5 \mathrm{Fe}$ & 93.80 & $2.0 / 2.7 / 3.5 / 4.6$ \\
\hline 2 & GXI $3 /-200$ & $11 \mathrm{Be} 0.5 \mathrm{Fe}$ & 93.80 & $2.0 / 2.7 / 3.5 / 4.6$ \\
\hline 3 & GXI $3 /-200$ & $11 \mathrm{Be} 0.5 \mathrm{Ti}$ & 93.80 & $2.2 / 2.45 / 2.7 / 2.95$ \\
\hline 4 & $\mathrm{FXI} /+250$ & $21 \mathrm{Be} 0.5 \mathrm{Ti}$ & 93.80 & $2.2 / 2.45 / 2.7 / 2.95$ \\
\hline 5 & FXI $/+150$ & $21 \mathrm{Be} 0.5 \mathrm{Sc}$ & 93.80 & $2.2 / 2.45 / 2.7 / 2.95$ \\
\hline 6 & GXI $2 /-300$ & $21 \mathrm{Be} 0.5 \mathrm{Fe}$ & 96.20 & $2.0 / 2.3 / 3.0 / 4.0$ \\
\hline 7 & GXI $2 /-300$ & $21 \mathrm{Be} 0.5 \mathrm{Fe}$ & 96.20 & $2.0 / 2.3 / 2.7 / 3.2$ \\
\hline 8 & GXI $2 /-300$ & $21 \mathrm{Be} 0.5 \mathrm{Ti}$ & 96.20 & $1.2 / 1.7 / 2.2 / 2.7$ \\
\hline 15 & GXI $3 /-100$ & $21 \mathrm{Be} 0.5 \mathrm{~V}$ & 93.80 & $4.2 / 4.95 / 5.7 / 6.45$ \\
\hline 14 & GXI $3 /-100$ & $41 \mathrm{Be} 0.5 \mathrm{Ti}$ & 95.70 & $2.8 / 3.55 / 4.3 / 5.05$ \\
\hline 13 & GXI $2 /-100$ & $41 \mathrm{Be} 0.5 \mathrm{Ti}$ & 95.70 & $1.5 / 1.9 / 2.3 / 2.7$ \\
\hline 11 & GXI $2 /-100$ & $41 \mathrm{Be} 0.5 \mathrm{Cu}$ & 95.70 & $1.2 / 1.5 / 1.8 / 2.1$ \\
\hline 12 & GXI $2 /-100$ & $41 \mathrm{Be} 0.5 \mathrm{Cu}$ & 95.70 & $2.2 / 2.4 / 3.2 / 3.6$ \\
\hline 9 & GXI $2 /-100$ & $41 \mathrm{Be} 0.5 \mathrm{Fe}$ & 95.70 & $1.8 / 2.05 / 2.3 / 2.55$ \\
\hline 10 & GXI $2 /-100$ & $41 \mathrm{Be} 0.5 \mathrm{Fe}$ & 95.70 & $3.5 / 3.9 / 4.3 / 5.0$ \\
\hline 16 & $\mathrm{FXI} /+150$ & $21 \mathrm{Be} 0.5 \mathrm{Sc}$ & 81.15 & $1.0 / 1.4 / 1.8 / 2.2$ \\
\hline 17 & FXI $/+150$ & $21 \mathrm{Be} 0.5 \mathrm{Sc}$ & 81.20 & $1.2 / 1.6 / 2.0 / 2.4$ \\
\hline 18 & FXI / +150 & $21 \mathrm{Be} 0.5 \mathrm{Sc}$ & 82.60 & $1.5 / 1.9 / 2.3 / 2.7$ \\
\hline 19 & FXI $/+150$ & $21 \mathrm{Be} 0.5 \mathrm{Sc}$ & 82.60 & $1.7 / 2.1 / 2.5 / 2.9$ \\
\hline 20 & FXI $/+150$ & $21 \mathrm{Be} 0.5 \mathrm{Fe}$ & 82.60 & $2.3 / 2.8 / 3.3 / 3.8$ \\
\hline 21 & FXI / +150 & $21 \mathrm{Be} 0.5 \mathrm{Fe}$ & 82.60 & $2.3 / 2.8 / 3.3 / 3.8$ \\
\hline 22 & FXI $/+150$ & $21 \mathrm{Be} 0.5 \mathrm{Fe}$ & 82.60 & $1.6 / 2.0 / 2.4 / 2.8$ \\
\hline
\end{tabular}


Table 4: Comments on SIM 4 Data

\begin{tabular}{|l|l|}
\hline Shotname & \multicolumn{1}{|c|}{ Comments on SIM 4 Data and Other Things. } \\
\hline 1 & No data on strip 1 - did not have batwing shielding. \\
\hline 2 & No data on strip 1 - did not have batwing shielding. \\
\hline 3 & Strip 3 did not fire. GXI was damaged from target debris. \\
\hline 4 & Weak images of the package - gain too low on FXI. \\
\hline 5 & Very weak images of the package - gain too low on FXI. \\
\hline 6 & $\begin{array}{l}\text { Strip 4 did not fire. Forced to use } 8 \text { X magnification pinholes with } 12 \mathrm{X} \\
\text { magnification snout and lost data on all the 1st pinholes. }\end{array}$ \\
\hline 7 & NOVA diagnostic trigger failed to fire. No data. \\
\hline 8 & $\begin{array}{l}\text { Strip 4 did not fire. Forced to use } 8 \text { X magnification pinholes with } 12 \mathrm{X} \\
\text { magnification snout and lost data on all the 1st pinholes. }\end{array}$ \\
\hline 15 & Laser did not fire properly - low energy. Strips 2 \& 4 did not fire. \\
\hline 14 & Strips 2 \& 4 did not fire. \\
\hline 13 & This was the null shot - no perturbations on package. \\
\hline 11 & Cu backlighter was dim. Package thickness different from requested. \\
\hline 12 & Cu backlighter was dim. \\
\hline 9 & Package thickness different from that requested. \\
\hline 10 & Shot went well. \\
\hline 16 & $\begin{array}{l}\text { Did not insert a Be filter built into FXI. Allowed light in and ruined } \\
\text { data. }\end{array}$ \\
\hline 17 & Strip 1 did not fire. \\
\hline $18,19,22$ & Shot went well. \\
\hline 20 & $\begin{array}{l}\text { Gain setting on FXI was too low for thicker packages. No data. Lost } \\
\text { data on the streak camera due to lead shielding falling on crystal. }\end{array}$ \\
\hline 21 & Diagnostic alignment off. Could only see cold side of target. \\
\hline
\end{tabular}


Table 5: SIM 3 and SIM 6

\begin{tabular}{|l|l|l|l|l|l|}
\hline $\begin{array}{c}\text { Shot- } \\
\text { name }\end{array}$ & $\begin{array}{c}\text { SIM 3 } \\
\text { Crystal }\end{array}$ & $\begin{array}{c}\text { SIM 3 Filter } \\
\text { (mil) }\end{array}$ & $\begin{array}{c}\text { SIM 3 TP/Cam } \\
\text { Delays (ns) }\end{array}$ & SIM 6 Filter & $\begin{array}{c}\text { SIM 6 TP/Cam } \\
\text { Delays (ns) }\end{array}$ \\
\hline 1 & PET & $11 \mathrm{Be} 0.5 \mathrm{Fe}$ & $67.70 / 3.3$ & $11 \mathrm{Be} 0.5 \mathrm{Fe}$ & $47.85 / 2.0$ \\
\hline 2 & PET & $11 \mathrm{Be} 0.5 \mathrm{Fe}$ & $67.70 / 3.3$ & $11 \mathrm{Be} 0.5 \mathrm{Fe}$ & $47.85 / 2.0$ \\
\hline 3 & RbAP & $11 \mathrm{Be} 0.5 \mathrm{Ti}$ & $67.70 / 3.5$ & $11 \mathrm{Be} 0.5 \mathrm{Ti}$ & $47.85 / 2.2$ \\
\hline 4 & RbAP & $11 \mathrm{Be} 0.5 \mathrm{Ti}$ & $67.70 / 3.5$ & $11 \mathrm{Be} 0.5 \mathrm{Ti}$ & $47.85 / 2.2$ \\
\hline 5 & RbAP & $11 \mathrm{Be} \mathrm{0.5} \mathrm{Sc}$ & $67.70 / 4.5$ & $11 \mathrm{Be} \mathrm{0.5} \mathrm{Sc}$ & $47.85 / 2.2$ \\
\hline 6 & PET & $11 \mathrm{Be} 0.5 \mathrm{Fe}$ & $67.70 / 3.3$ & $11 \mathrm{Be}+\mathrm{Fe}$ & $43.95 / 2.0$ \\
\hline 7 & PET & $11 \mathrm{Be} 0.5 \mathrm{Fe}$ & $67.70 / 3.3$ & $11 \mathrm{Be}+\mathrm{Fe}$ & $43.95 / 2.0$ \\
\hline 8 & RbAP & $11 \mathrm{Be} 0.5 \mathrm{Ti}$ & $67.70 / 2.0$ & $21 \mathrm{Be}$ & $43.95 / 1.2$ \\
\hline 15 & RbAP & $0.5 \mathrm{Ti} 0.5 \mathrm{~V}$ & $67.70 / 5.5$ & $41 \mathrm{Be}$ & $46.35 / 4.1$ \\
\hline 14 & RbAP & $11 \mathrm{Be} 0.5 \mathrm{Ti}$ & $67.70 / 4.1$ & $41 \mathrm{Be}$ & $46.35 / 2.7$ \\
\hline 13 & RbAP & $11 \mathrm{Be} 0.5 \mathrm{Ti}$ & $67.70 / 2.3$ & $41 \mathrm{Be}$ & $43.90 / 1.5$ \\
\hline 11 & PET & $11 \mathrm{Be} 0.5 \mathrm{Cu}$ & $67.70 / 2.0$ & $41 \mathrm{Be}$ & $43.90 / 1.2$ \\
\hline 12 & PET & $11 \mathrm{Be} 0.5 \mathrm{Cu}$ & $67.70 / 3.0$ & $41 \mathrm{Be}$ & $43.90 / 2.2$ \\
\hline 9 & PET & $11 \mathrm{Be} 0.5 \mathrm{Fe}$ & $67.70 / 2.3$ & $\mathrm{Fe}$ & $43.90 / 1.8$ \\
\hline 10 & PET & $11 \mathrm{Be} 0.5 \mathrm{Fe}$ & $67.70 / 4.2$ & $\mathrm{Fe}$ & $43.90 / 3.5$ \\
\hline 16 & RbAP & $11 \mathrm{Be} 0.5 \mathrm{Ti}$ & $67.74 / 0.8$ & $21 \mathrm{Be}$ & $46.35 / 1.0$ \\
\hline 17 & RbAP & $11 \mathrm{Be} 0.5 \mathrm{Ti}$ & $67.74 / 1.0$ & $21 \mathrm{Be}$ & $46.35 / 1.2$ \\
\hline 18 & RbAP & $11 \mathrm{Be} 0.5 \mathrm{Ti}$ & $67.74 / 2.3$ & $21 \mathrm{Be}$ & $46.35 / 1.2$ \\
\hline 19 & RbAP & $11 \mathrm{Be} 0.5 \mathrm{Ti}$ & $67.74 / 2.3$ & $21 \mathrm{Be}$ & $46.35 / 1.7$ \\
\hline 20 & PET & $11 \mathrm{Be} 0.5 \mathrm{Fe}$ & $67.74 / 3.0$ & $21 \mathrm{Be}$ & $46.35 / 2.0$ \\
\hline 21 & PET & $11 \mathrm{Be} 0.5 \mathrm{Fe}$ & $67.74 / 3.3$ & $21 \mathrm{Be}$ & $46.35 / 2.5$ \\
\hline 22 & PET & $11 \mathrm{Be} 0.5 \mathrm{Fe}$ & $67.74 / 2.6$ & $21 \mathrm{Be}$ & $46.35 / 1.6$ \\
\hline
\end{tabular}


Table 6: Backlighter

\begin{tabular}{|c|c|c|c|c|}
\hline $\begin{array}{l}\text { Shot- } \\
\text { name }\end{array}$ & $\begin{array}{l}\text { Backlighter \& } \\
\text { Thickness }(\mu \mathrm{m})\end{array}$ & $\begin{array}{l}\text { Backlighter } \\
\text { He- } \alpha(\mathrm{keV})\end{array}$ & $\begin{array}{l}\text { Backlighter Delay/ } \\
\text { Pulse length (ns) }\end{array}$ & $\begin{array}{c}\text { Energy on } \\
\text { Backlighter }(\mathrm{kJ})\end{array}$ \\
\hline 1 & $18 \mathrm{Fe}$ & 6.7 & $1.8 / 3$ & 8.67 \\
\hline 2 & $25 \mathrm{Fe}$ & 6.7 & $2.0 / 3$ & 8.88 \\
\hline 3 & $25 \mathrm{Ti}$ & 4.7 & $1.5 / 3$ & 9.87 \\
\hline 4 & $25 \mathrm{Ti}$ & 4.7 & $1.5 / 3$ & 8.76 \\
\hline 5 & Sc & 4.3 & $1.5 / 3$ & 9.16 \\
\hline 6 & $18 \mathrm{Fe}$ & 6.7 & $1.8 / 3$ & 9.15 \\
\hline 7 & $25 \mathrm{Mn}$ & 6.2 & $1.8 / 2$ & 9.65 \\
\hline 8 & $25 \mathrm{Sc}$ & 4.3 & $1.0 / 2$ & 10.82 \\
\hline 15 & $13 \mathrm{~V}$ & 5.2 & $4.0 / 3$ & 7.45 \\
\hline 14 & $25 \mathrm{Sc}$ & 4.3 & $2.6 / 3$ & 6.93 \\
\hline 13 & $25 \mathrm{Sc}$ & 4.3 & $1.3 / 2$ & 8.04 \\
\hline 11 & $50 \mathrm{Cu}$ & 8.3 & $1.0 / 2$ & 7.59 \\
\hline 12 & $50 \mathrm{Cu}$ & 8.3 & $2.0 / 2$ & 8.15 \\
\hline 9 & $18 \mathrm{Fe}$ & 6.7 & $1.3 / 2$ & 8.02 \\
\hline 10 & $18 \mathrm{Fe}$ & 6.7 & $2.0 / 2$ & 7.74 \\
\hline 16 & $25 \mathrm{Sc}$ & 4.3 & $0.8 / 2.0$ & \\
\hline 17 & $25 \mathrm{Sc}$ & 4.3 & $1.0 / 2.0$ & 9.44 \\
\hline 18 & $16 \mathrm{Sc}$ & 4.3 & $1.3 / 2.0$ & 9.82 \\
\hline 19 & $16 \mathrm{Sc}$ & 4.3 & $1.5 / 2.0$ & 8.93 \\
\hline 20 & $\mathrm{Mn}$ & 6.2 & $2.1 / 2.0$ & \\
\hline 21 & $18 \mathrm{Fe}$ & 6.7 & $2.1 / 2.0$ & 9.33 \\
\hline 22 & $18 \mathrm{Fe}$ & 6.7 & $1.4 / 2.0$ & 8.94 \\
\hline
\end{tabular}


Table 7: Laser Beam and Diagnostic Alignment for all Shots

\begin{tabular}{|l|l|l|l|l|}
\hline $\begin{array}{c}\text { Beam Line or } \\
\text { Diagnostic }\end{array}$ & $\begin{array}{c}\mathrm{X}(-\mathrm{N} / \mathrm{+S}) \\
(\mu \mathrm{m})\end{array}$ & $\begin{array}{c}\mathrm{Y}(-\mathrm{E} /+\mathrm{W}) \\
(\mu \mathrm{m})\end{array}$ & $\begin{array}{c}\text { Z(-Up/ } \\
+ \text { Down }) \\
(\mu \mathrm{m})\end{array}$ & $\begin{array}{c}\text { Lens/Z(- } \\
\text { Div/+Con }) \\
(\mu \mathrm{m})\end{array}$ \\
\hline Beams 1,3,5,9 & 0 & 1375 & 0 & -1000 \\
\hline Beams 2,4,6,10 & 0 & -1375 & 0 & -1000 \\
\hline $\begin{array}{l}\text { Backlighter } \\
\text { Beams 7,8 Face- } \\
\text { on }\end{array}$ & 3800 & 0 & 1236 & 0 \\
\hline $\begin{array}{l}\text { Backlighter } \\
\text { Beams 7,8 Side- } \\
\text { on }\end{array}$ & 4074 & 0 & 404 & 0 \\
\hline $\begin{array}{l}\text { SIM 4 \& 6 Face- } \\
\text { on }\end{array}$ & 3800 & 0 & 1236 & N/A \\
\hline $\begin{array}{l}\text { SIM 4 \& 6 Side- } \\
\text { on }\end{array}$ & 4074 & 0 & 404 & N/A \\
\hline SIM 3 Face-on & 3800 & 0 & 1680 & N/A \\
\hline SIM 3 Side-on & 4074 & 0 & 848 & N/A \\
\hline Target & 0 & 0 & 0 & N/A \\
\hline
\end{tabular}

Target rotation was $0(-\mathrm{CCW} /+\mathrm{CW})$. 


\section{Bibliography}

Baltrusaitis, R. M. et al. Sept. 1996 "Simulation of Shock Generated Instabilities," Physics of Fluids $\underline{8}$ (9): 2471-2483.

Bel'kov, S. A. et al. 1998 "Simulation of Rayleigh-Taylor Instability Growth Rate of Laser-Accelerated Plane Targets," submitted to Physics of Plasmas.

Bird, R. B., Stewart, W. E., and Lightfoot, E. N. 1960 Transport Phenomena. John Wiley \& Sons New York, NY.

Byrne, N., Betlach, T., Gittings, M. L., April 28-30, 1992 "RAGE: A 2D Adaptive Grid Eulerian Nonequilibrium Radiation Code," Proceedings of the Defense Nuclear Agency Numerical Methods Symposium.

Duderstadt, J. and Moses, G. 1982 Inertial Confinement Fusion. New York, NY: John Wiley and Sons, presently out of print.

Gossard, Earl and Hooke, William. 1975 Waves in the Atmosphere. Elsevier Scientific New York, NY.

Gittings, M. L., April 28-30, 1992 "SAIC's Adaptive Grid Eulerian Hydrocode," Proceedings of the Defense Nuclear Agency Numerical Methods Symposium

Haan, S. W. June 1991 "Hydrodynamic Instabilities on ICF Capsules," UCRL-JC107592. Lawrence Livermore National Laboratories, Livermore, CA.

Haan, S. W. August 1991 "Weakly Nonlinear Hydrodynamic Instabilities in Inertial Fusion," Physics of Fluids B 3(8): 2349-2355.

Hoffman, Nelson M. Aug. 1994 "Hydrodynamic Instabilities in Inertial Confinement Fusion," LAUR-94-3945. Los Alamos National Laboratory, Los Alamos, NM.

Hoffman, Nelson M. 1997 Staff member, MS F663, Los Alamos National Laboratory, Los Alamos, NM 87545. Personal communication.

Hollowell, D., et al. June 1-5, 1997 "Ablative Rayleigh-Taylor Instability Modeling," Proceedings of the 27th Annual Anomalous Absorption Conference, University of British Columbia, Vancouver, B. C.

Hogan, W., Bangerter, R., and Kulcinski, G. Sept. 1992 "Energy from Inertial 
Fusion," Physics Today 42-50.

Holmes, R. L., Grove, J. W., Sharp, D. H. 1995 “Numerical Investigation of Richtmyer-Meshkov Instability Using Front Tracking," Lournal of Fluid Mechanics 301: 51-64.

Krall and Trivelpiece 1986 Principles of Plasma Physics. San Francisco, CA: San Francisco Press, Inc.

Krauser, W. et al. 1996 "Ignition Target Design and Robustness Studies for the National Ignition Facility," Physics of Plasmas 3 (5): 2084-2093.

Lindl, J., McCrory, R. L., Cambell, E. M. Sept. 1992 “Progress Toward Ignition and Burn Propagation in Inertial Confinement Fusion," Physics Today 3240 .

Mikaelian, Karnig Oct. 1982 "Rayleigh-Taylor Instabilities in Stratified Fluids," Physical Review A 26 (4): 2140-2158.

Mikaelian, Karnig Sept. 1983 "Time Evolution of Density Perturbations in Accelerating, Stratified Fluids," Physical Review A 28 (3): 1637-1646.

Mikaelian, Karnig Jan. 1985 "Richtmyer-Meshkov Instabilities in Stratified Fluids," Physical Review A 31 (1): 410-419

Mikaelian, Karnig April 1995 "Rayleigh-Taylor and Richtmyer-Meshkov Instabilities in Finite Thickness Fluid Layers," Physics of Fluids Z (4): 888-890.

Mikaelian, Karnig May 1996 "Numerical Simulation of Richtmyer-Meshkov Instabilities in finite-Thickness Fluid Layers," Physics of Fluids $\underline{8}$ (5): 1269-1291.

Ofer, D. et al. 1996 "Modal Model for the Nonlinear Multimode Rayleigh-Taylor Instability," Physics of Plasmas 3(8): 3073-3089.

Ott, Edward, Nov. 1972 "Nonlinear Evolution of the Rayleigh-Taylor Instability of a Thin Layer," Physical Review Letters 29 (21): 1429-1432.

Remington, B. A., et al. April 1992 "Large Growth, Planar Rayleigh-Taylor experiments on NOVA," Physics of Fluids B 4 (4): 967-978.

Remington, B. A., et al. July 1993 “Laser-Driven Hydrodynamic Instability Experiments," Physics of Fluids B $\underline{5}$ (7): 2589-2595. 
Richtmyer, Robert 1960 "Taylor Instability in Shock Acceleration of Compressible Fluids," Communications on Pure and Applied Mathematics, vol. XIII, 297-319.

Scannapieco, A. Sept. 1981 "Atmospheric Type Modes in Laser Fusion Targets," Physics of Fluids 24 (9): 1699-1705.

Schappert, G., et al. 1996 "Planar Rayleigh-Taylor Instability Growth in Copper Foils," Proceedings of the 38th Annual Meeting of the Division of Plasma Physics Nov 11-15, Denver, Co.

Takabe, H., et al. 1985 "Self-Consistent Growth Rate of the Rayleigh-Taylor Instability in an Ablatively Accelerating Plasma," Physics of Fluids 28: 3676.

Weber, S. V., Remington, B. A. et al. Nov 1994 "Modeling of NOVA Indirect Drive . Rayleigh-Taylor Experiments," Physics of Plasmas 1 (11): 3652-3661.

Yang, Yumin and Zhang, Qiang May 1993 "General Properties of a Multilayer Stratified Fluids System," Physics of Fluids A 5 (5): 1167-1181.

Yang, Y., Zhang, Q., Sharp, D. 1994 "Small Amplitude Theory of Richtmyer-Meshkov Instability," Physics of Fluids 6: 1856-1873.

Youngs, David L. 1984 “Numerical Simulation of Turbulent Mixing by RayleighTaylor Instability," Physica 12D: 32-44.

Zel'dovich and Razer 1966 Physics of Shock Waves and High Temperature Hydrodynamic Phenomena Volume I, Academic Press, New York, NY.

Zimmerman, G. B. and Kruer, W. L. 1975 "Numerical Simulation of Laser-Initiated Fusion," Comments on Plasma Physics and Controlled Fusion $\underline{\underline{2}}$ (2): 51-61. 